University of Texas Rio Grande Valley

ScholarWorks @ UTRGV

Physics and Astronomy Faculty Publications

and Presentations

College of Sciences

$1-1-2009$

\title{
Stellar population models and individual element abundances. II. Stellar spectra and integrated light models
}

Hyun Chul Lee

Guy Worthey

Aaron Dotter

Brian Chaboyer

Darko Jevremović

See next page for additional authors

Follow this and additional works at: https://scholarworks.utrgv.edu/pa_fac

Part of the Astrophysics and Astronomy Commons

\section{Recommended Citation}

Hyun Chul Lee, et. al., (2009) Stellar population models and individual element abundances. II. Stellar spectra and integrated light models.Astrophysical Journal694:2902. DOI: http://doi.org/10.1088/ 0004-637X/694/2/902

This Article is brought to you for free and open access by the College of Sciences at ScholarWorks @ UTRGV. It has been accepted for inclusion in Physics and Astronomy Faculty Publications and Presentations by an authorized administrator of ScholarWorks @ UTRGV. For more information, please contact justin.white@utrgv.edu, william.flores01@utrgv.edu. 


\section{Authors}

Hyun Chul Lee, Guy Worthey, Aaron Dotter, Brian Chaboyer, Darko Jevremović, E. Baron, Michael M. Briley, Jason W. Ferguson, Paula Coelho, and Scott C. Trager 


\title{
STELLAR POPULATION MODELS AND INDIVIDUAL ELEMENT ABUNDANCES. II. STELLAR SPECTRA AND INTEGRATED LIGHT MODELS
}

\author{
Hyun-chul Lee ${ }^{1}$, Guy Worthey ${ }^{1}$, Aaron Dotter ${ }^{2}$, Brian Chaboyer ${ }^{2}$, Darko Jevremović ${ }^{3,4}$, E. Baron ${ }^{4}$, \\ Michael M. Briley ${ }^{5}$, Jason W. Ferguson ${ }^{6}$, Paula Coelho $^{7}$, and Scott C. Trager ${ }^{8}$ \\ ${ }^{1}$ Department of Physics and Astronomy, Washington State University, Pullman, WA 99164-2814, USA; hclee@wsu.edu, gworthey@wsu.edu \\ ${ }^{2}$ Department of Physics and Astronomy, Dartmouth College, 6127 Wilder Laboratory, Hanover, NH 03755, USA \\ ${ }^{3}$ Astronomical Observatory, Volgina 7, 11160 Belgrade, Serbia and Montenegro \\ ${ }^{4}$ Homer L. Dodge Department of Physics and Astronomy, University of Oklahoma, 440 West Brooks, Room 110, Norman, OK 73019-2061, USA \\ ${ }^{5}$ Department of Physics and Astronomy, University of Wisconsin, Oshkosh, 800 Algoma Boulevard, Oshkosh, WI 54901, USA \\ ${ }^{6}$ Department of Physics, Wichita State University, Wichita, KS 67260-0032, USA \\ ${ }^{7}$ Institut d'Astrophysique, Centre National de la Recherche Scientifique, Université Pierre et Marie Curie, 98 bis Bd Arago, 75014 Paris, France \\ ${ }^{8}$ Kapteyn Astronomical Institute, University of Groningen, P.O. Box 800, 9700 Avenue Groningen, Netherlands \\ Received 2008 March 6; accepted 2008 December 30; published 2009 March 20
}

\begin{abstract}
The first paper in this series explored the effects of altering the chemical mixture of the stellar population on an element-by-element basis on stellar evolutionary tracks and isochrones to the end of the red giant branch. This paper extends the discussion by incorporating the fully consistent synthetic stellar spectra with those isochrone models in predicting integrated colors, Lick indices, and synthetic spectra. Older populations display element ratio effects in their spectra at higher amplitude than younger populations. In addition, spectral effects in the photospheres of stars tend to dominate over effects from isochrone temperatures and lifetimes, but, further, the isochrone-based effects that are present tend to fall along the age-metallicity degeneracy vector, while the direct stellar spectral effects usually show considerable orthogonality.
\end{abstract}

Key words: stars: abundances - stars: evolution

Online-only material: color figures

\section{INTRODUCTION}

Little is known about the influence of individual element abundances on the integrated flux of a stellar population. As present, it is impossible to derive an accurate age within $10 \%$ from the integrated light of a clusterlike single-age and single-abundance stellar population, and the primary reason is the complication due to abundance ratio effects (Worthey 1998). The secondary reason is that the input ingredients and choices made in even the most sophisticated stellar evolution calculations induce scatter in the results (Charlot et al. 1996). The tertiary reason is the set of difficulties associated with stellar flux knowledge, such as colors, line strengths, or spectra, that are needed at each stellar evolutionary phase to represent the component stars. Additional uncertainties exist, such as dust extinction, stellar rotation and activity, the blue straggler frequency, and the effects of close binaries.

Dotter et al. (2007b, hereafter Paper I), explored the effects of 12 chemical mixtures on the $L, T_{\text {eff }}$, and lifetimes of stars along stellar evolutionary isochrones and upon the opacities needed to calculate the stellar models. The mixtures explored were solar, $\alpha$-element enhanced, and 10 cases where only one element at a time was enhanced, for elements $\mathrm{C}, \mathrm{N}, \mathrm{O}, \mathrm{Ne}, \mathrm{Mg}, \mathrm{Si}, \mathrm{S}, \mathrm{Ca}, \mathrm{Ti}$, and $\mathrm{Fe}$. The mixtures were re-scaled so that the mass fraction of heavy elements, $Z$, was constant. ${ }^{9}$

The conclusions from that paper could be summarized by splitting the elements that were investigated into three categories. "Displacers" include O, C, N, and Ne. These are elements that are abundant but supply less opacity per unit mass

\footnotetext{
9 Added to these 12 mixtures were three at nonconstant $Z$ and variables $X$ and $Y$ but constant $[\mathrm{Fe} / \mathrm{H}]$ with 0.2 dex more carbon, 0.3 dex more nitrogen, and 0.3 dex more oxygen (see Dotter et al. 2007b).
}

than heavier elements. At fixed $Z$, boosting a displacer element will therefore decrease the opacity. This leads to shorter stellar lifetimes and hotter stars. "Boosters" include $\mathrm{Mg}$ and $\mathrm{Si}$. These elements are good opacity sources, so that boosting a booster will increase opacity and make cooler stars that live somewhat longer. "Oddball" elements defy these trends, and include $\mathrm{Ca}$ and $\mathrm{Ti}$, both of which make cooler stars, that, nevertheless, have shorter lifetimes. Another oddball is S, which decreases low-temperature opacity but increases high-temperature opacity so that its ultimate effect on temperatures and lifetimes is small. An element worth special mention is Fe, because its high-temperature opacity has considerable structure. One of its opacity peaks corresponds to the temperature characteristics of the outer edge of the convective core that develops in stars slightly more massive than the Sun. Increasing the Fe abundance therefore strongly couples to the onset of convective core overshooting effects, and leads to a strong luminosity effect in the region of the main sequence turnoff. The temperature, luminosity, and lifetime effects are illustrated in Paper I, but further consequences will be illuminated in this paper.

Besides isochrones, the other major ingredient in any population synthesis effort is some representation of stellar flux for each star in the isochrone. The effects of individual element abundances on the spectra of stars have been studied for years, and enormous progress has been made in finding the structure of stellar atmospheres and calculating the emergent flux. The two most cited programs for constructing model atmospheres of stars are ATLAS (Kurucz 1970) and MARCS (Gustafsson et al. 1975), and we use results from these codes in this work. For spectral synthesis of the emergent flux, we use SYNTHE (Kurucz 1970), FANTOM (Coelho et al. 2005; Cayrel et al. 1991), and SSG (Bell et al. 1994). For ordinary population synthesis, empirical spectra or colors can be used (Vazdekis 1999), 
Table 1

Gross Color Behavior with Metallicity Increase

\begin{tabular}{|c|c|c|c|c|c|c|c|}
\hline Color & $\begin{array}{l}\text { Synthetic } \\
\text { Color } \\
\text { (mag) }\end{array}$ & $\begin{array}{c}\Delta \text { Color, } \\
24 \text { Elements } \\
\text { Enhanced } \\
(\mathrm{mmag})\end{array}$ & $\begin{array}{c}\Delta \text { Color, } \\
\text { All Elements } \\
\text { Enhanced } \\
(\mathrm{mmag})\end{array}$ & $\begin{array}{c}\Delta \text { Color } \\
T_{\text {eff }} \\
\text { (mmag) }\end{array}$ & $\begin{array}{l}\text { Empirical } \\
\text { Color } \\
(\mathrm{mag})\end{array}$ & $\begin{array}{c}\Delta \text { Color, } \\
\text { All Elements } \\
\text { Enhanced } \\
(\mathrm{mmag})\end{array}$ & $\begin{array}{c}\Delta \text { Color, } \\
T_{\text {eff }} \\
(\mathrm{mmag})\end{array}$ \\
\hline \multicolumn{8}{|c|}{ Dwarf, $T_{\text {eff }}=4000 \mathrm{~K}$} \\
\hline$U-B$ & 1.26 & 21 & 81 & -106 & $1.36 \pm 0.07$ & 58 & -85 \\
\hline$B-V$ & 1.43 & 43 & 35 & -137 & $1.36 \pm 0.02$ & 22 & -81 \\
\hline$V-R$ & 0.89 & 16 & 24 & -94 & $0.82 \pm 0.01$ & 3 & -74 \\
\hline$V-I$ & 1.60 & 13 & 24 & -176 & $1.58 \pm 0.02$ & -5 & -176 \\
\hline \multicolumn{8}{|c|}{ Dwarf, $T_{\text {eff }}=5000 \mathrm{~K}$} \\
\hline$U-B$ & 0.61 & 123 & 111 & -179 & $0.71 \pm 0.07$ & 160 & -169 \\
\hline$B-V$ & 0.91 & 55 & 45 & -90 & $0.96 \pm 0.02$ & 62 & -101 \\
\hline$V-R$ & 0.52 & 21 & 19 & -62 & $0.52 \pm 0.01$ & 11 & -55 \\
\hline$V-I$ & 0.98 & 15 & 14 & -103 & $0.96 \pm 0.02$ & 13 & -97 \\
\hline \multicolumn{8}{|c|}{ Dwarf, $T_{\text {eff }}=6000 \mathrm{~K}$} \\
\hline$U-B$ & 0.02 & 113 & 95 & -33 & $0.16 \pm 0.07$ & 114 & -75 \\
\hline$B-V$ & 0.58 & 39 & 37 & -50 & $0.61 \pm 0.02$ & 42 & -70 \\
\hline$V-R$ & 0.33 & 15 & 11 & -28 & $0.34 \pm 0.01$ & 4 & -33 \\
\hline$V-I$ & 0.66 & 16 & 5 & -55 & $0.65 \pm 0.01$ & 1 & -60 \\
\hline \multicolumn{8}{|c|}{ Giant,$T_{\mathrm{eff}}=4000 \mathrm{~K}$} \\
\hline$U-B$ & 1.34 & 115 & 137 & -182 & $1.69 \pm 0.07$ & 52 & -194 \\
\hline$B-V$ & 1.45 & 39 & 57 & -166 & $1.44 \pm 0.02$ & 22 & -103 \\
\hline$V-R$ & 0.83 & 5 & 21 & -120 & $0.79 \pm 0.01$ & 5 & -82 \\
\hline$V-I$ & 1.51 & -4 & 20 & -198 & $1.51 \pm 0.02$ & 4 & -182 \\
\hline \multicolumn{8}{|c|}{ Giant,$T_{\mathrm{eff}}=5000 \mathrm{~K}$} \\
\hline$U-B$ & 0.57 & 159 & 147 & -159 & $0.77 \pm 0.07$ & 161 & -194 \\
\hline$B-V$ & 0.93 & 52 & 60 & -107 & $0.97 \pm 0.02$ & 63 & -116 \\
\hline$V-R$ & 0.48 & 18 & 12 & -46 & $0.49 \pm 0.01$ & 10 & -52 \\
\hline$V-I$ & 0.92 & 9 & -1 & -80 & $0.94 \pm 0.01$ & 11 & -87 \\
\hline \multicolumn{8}{|c|}{ Giant,$T_{\text {eff }}=6000 \mathrm{~K}$} \\
\hline$U-B$ & 0.16 & 79 & 83 & 19 & $0.11 \pm 0.07$ & 109 & -81 \\
\hline$B-V$ & 0.55 & 48 & 54 & -64 & $0.55 \pm 0.02$ & 41 & -85 \\
\hline$V-R$ & 0.31 & 12 & 9 & -35 & $0.31 \pm 0.01$ & 4 & -36 \\
\hline$V-I$ & 0.61 & 12 & 4 & -69 & $0.64 \pm 0.01$ & 1 & -70 \\
\hline
\end{tabular}

Notes. 1. The "Dwarf" islog $g=4.5$, and the "Giant" islog $g=2.5$. 2. Column 2 presents the calculations at $[\mathrm{R} / \mathrm{H}]=0$, where $\mathrm{R}$ is the generic heavy element (see Section 2.2). 3. Column 3 presents the case where 24 elements (C, N, O, Na, Mg, Al, Si, S, Cl, K, Ca, Sc, Ti, V, Cr, Mn, $\mathrm{Fe}, \mathrm{Co}, \mathrm{Ni}, \mathrm{Cu}, \mathrm{Zn}, \mathrm{Sr}, \mathrm{Ba}, \mathrm{Eu})$ are enhanced by 0.3 dex. 4 . Columns 4 and 7 present the calculations at $[\mathrm{R} / \mathrm{H}]=0.3$ dex. 5 . Columns 5 and 8 present the color changes when the temperature is increased by $250 \mathrm{~K}$, synthetic and empirical, respectively.

but for investigating element-by-element effects, using synthetic spectra is clearly the way forward. Investigations into the effects of individual elements on stellar spectra, but with the intent of applying the results to galaxy spectra, include Tripicco \& Bell (1995) and Korn et al. (2005), who gauged the effects of 10 individual elements on the Lick system of 25 pseudo-equivalent width indices (Worthey et al. 1994; Worthey \& Ottaviani 1997). Serven et al. (2005) investigated 24 elements in spectra that ran from $3500 \AA$ to $9000 \AA$ with velocity smoothing appropriate for dynamically hot systems such as elliptical galaxies.

The present paper combines the new isochrones described in Paper I with greatly extended and updated synthetic spectra in order to obtain ab initio population synthesis models as a function of individual element abundances. ${ }^{10}$ All parts of the models, including high-temperature and low-temperature opacities, stellar evolutionary models, and synthetic spectra, include the altered abundances in the same way. At present the grid includes only solar $Z$, but calculations are underway to extend to many different abundances. This paper describes the new spectra and their color index results in Section 2, Lick index

\footnotetext{
${ }^{10}$ Coelho et al. (2007) did a similar investigation with a flat enhancement of
} all the $\alpha$-elements over a range of metallicity. results in Section 3, concluding with a discussion and summary section.

\section{DESCRIPTION OF NEW MODEL INGREDIENTS}

The new isochrones were described in Paper I, and we refer the reader to that paper for details. Twelve chemical mixtures were explored self-consistently with both high-temperature and low-temperature opacities adjusted properly, and evolution is complete only to the end of the red giant branch. It should be emphasized that the models are thus incomplete and should not be used blindly when comparing to real stellar populations until the helium-burning phases are properly incorporated. ${ }^{11}$ This is planned for the ongoing improvement of these models. In careful, differential ways, the present models give us important pointers to the behavior of stellar populations with variable element mixtures, but the absolute values of colors and indices are not to be trusted. The mixtures explored were solar, $\alpha$ element enhanced, and 10 cases where only one element at a time was enhanced ( $\mathrm{C}, \mathrm{N}, \mathrm{O}, \mathrm{Ne}, \mathrm{Mg}, \mathrm{Si}, \mathrm{S}, \mathrm{Ca}, \mathrm{Ti}$, and $\mathrm{Fe}$ ).

\footnotetext{
11 The contribution from the later stellar evolutionary phases to the spectral
} indices can be inferred from Figure 8 in Coelho et al. (2007). 
Table 2

Color Behavior with Enhancements at Constant Z

\begin{tabular}{|c|c|c|c|c|c|c|c|c|c|c|c|}
\hline Color & $\mathrm{C}$ & $\mathrm{N}$ & $\mathrm{O}$ & $\mathrm{Ne}$ & $\mathrm{Mg}$ & $\mathrm{Si}$ & $\mathrm{S}$ & $\mathrm{Ca}$ & $\mathrm{Ti}$ & $\mathrm{Fe}$ & $\alpha$ \\
\hline \multicolumn{12}{|c|}{ Dwarf, $T_{\text {eff }}=4000 \mathrm{~K}$} \\
\hline$U-B$ & -13 & 8 & 19 & -2 & -66 & -36 & 0 & -40 & 15 & 136 & -103 \\
\hline$B-V$ & 17 & -2 & -50 & -4 & -19 & 35 & 0 & -7 & 15 & 22 & -33 \\
\hline$V-R$ & -2 & -1 & -7 & -1 & 31 & -1 & 0 & -14 & 4 & 12 & 8 \\
\hline$V-I$ & -5 & -1 & -5 & -1 & 31 & -1 & 0 & -14 & 4 & 14 & 10 \\
\hline \multicolumn{12}{|c|}{ Dwarf, $T_{\text {eff }}=5000 \mathrm{~K}$} \\
\hline$U-B$ & 13 & 18 & -61 & -13 & -26 & -39 & -2 & -4 & 11 & 61 & -120 \\
\hline$B-V$ & 17 & 1 & -40 & -6 & -32 & 5 & -1 & 6 & 7 & 18 & -58 \\
\hline$V-R$ & -3 & -5 & -8 & -2 & 8 & -2 & 0 & 0 & 3 & 10 & -3 \\
\hline$V-I$ & -12 & -11 & 0 & -1 & 10 & -1 & 0 & -1 & 3 & 15 & 7 \\
\hline \multicolumn{12}{|c|}{ Dwarf, $T_{\text {eff }}=6000 \mathrm{~K}$} \\
\hline$U-B$ & -4 & 10 & -48 & -13 & 5 & -19 & -2 & 1 & 8 & 30 & -59 \\
\hline$B-V$ & 8 & 1 & -20 & -4 & -14 & -7 & 0 & 6 & 5 & 11 & -34 \\
\hline$V-R$ & 0 & -1 & -7 & -1 & 0 & -1 & 0 & 0 & 2 & 7 & -9 \\
\hline$V-I$ & 0 & -3 & -8 & -1 & 0 & 0 & 0 & 0 & 2 & 10 & -10 \\
\hline \multicolumn{12}{|c|}{ Giant, $T_{\text {eff }}=4000 \mathrm{~K}$} \\
\hline$U-B$ & 7 & 11 & -50 & -13 & -26 & -50 & -2 & -20 & 22 & 112 & -129 \\
\hline$B-V$ & 17 & -1 & -47 & -4 & -30 & 37 & 0 & -3 & 12 & 19 & -37 \\
\hline$V-R$ & -10 & -5 & 2 & 0 & 21 & 0 & 0 & -8 & 3 & 13 & 15 \\
\hline$V-I$ & -21 & -9 & 14 & 1 & 23 & 0 & 0 & -9 & 2 & 17 & 29 \\
\hline \multicolumn{12}{|c|}{ Giant, $T_{\text {eff }}=5000 \mathrm{~K}$} \\
\hline$U-B$ & 10 & 23 & -74 & -17 & 24 & -39 & -3 & 6 & 11 & 19 & -82 \\
\hline$B-V$ & 18 & 5 & -34 & -6 & -22 & -6 & -1 & 8 & 6 & 8 & -52 \\
\hline$V-R$ & 1 & -10 & -8 & -1 & 1 & 0 & 0 & 0 & 2 & 9 & -6 \\
\hline$V-I$ & -7 & -23 & 1 & 0 & 6 & 2 & 0 & 0 & 3 & 16 & 10 \\
\hline \multicolumn{12}{|c|}{ Giant, $T_{\text {eff }}=6000 \mathrm{~K}$} \\
\hline$U-B$ & -5 & 6 & -34 & -9 & 12 & -9 & -2 & 6 & 8 & 9 & -25 \\
\hline$B-V$ & 1 & 0 & -23 & -5 & -5 & -3 & -1 & 9 & 5 & 9 & -23 \\
\hline$V-R$ & 0 & 0 & -5 & -1 & -1 & 0 & 0 & 0 & 2 & 5 & -7 \\
\hline$V-I$ & 0 & -1 & -6 & -1 & 0 & 0 & 0 & -1 & 2 & 7 & -9 \\
\hline
\end{tabular}

Note. 1. All elements scaled individually by +0.3 dex, except $C$, which is increased by +0.15 dex.

The mixtures were re-scaled so that the mass fraction of heavy elements, $Z$, was held constant.

\subsection{Synthetic Spectra}

New for the present work is a collection of synthetic spectra from three different sources. Spectra for the coolest stars, at 3000 and $3190 \mathrm{~K}$ for $\log g=0$, were calculated using the FANTOM synthesis code and Plez (1992) model atmospheres. At 3500 and $3750 \mathrm{~K}$ for $\log g=0$ and 5 spectra were calculated using the FANTOM synthesis code and ATLAS model atmospheres exactly as described in Coelho et al. (2005), from $3000 \AA$ to $10000 \AA$ in $0.02 \AA$ steps. Spectra for the medium-temperature stars were calculated using the SSG synthesis code and MARCS model atmospheres from $3000 \AA$ to $10000 \AA$ in $0.01 \AA$ steps. There were nine temperatures in this range, from $4000 \mathrm{~K}$ to $6000 \mathrm{~K}$, in $250 \mathrm{~K}$ steps. Gravities of $\log g=4.5$ and 2.5 were used for all nine temperatures, but $\log g=0.5$ was included for the three coolest. Spectra for hot stars, at $7000 \mathrm{~K}, 8000 \mathrm{~K}$, $10000 \mathrm{~K}$, and $20000 \mathrm{~K}$, were calculated using the SYNTHE synthesis code starting from ATLAS model atmospheres in high resolution with logarithmic wavelength spacings. Gravities of $\log g=4.5$ and 2.5 were used except the $20000 \mathrm{~K}$ models, where 4.5 and 3.0 were used. The lines lists were not completely homogeneous between the three regimes, although they share much in common. The hot stars were computed with R. Kurucz (kurucz.harvard.edu) line lists, the medium-temperature stars included custom modifications by R. Bell, M. Tripicco, and
M. Houdashelt, and the cool stars benefited from the TiO rescalings described in Coelho et al. (2005).

These spectra were then rebinned to a common wavelength range of 3000-10000 $\AA$ and a common linear wavelength binning of $0.01 \AA$ per flux point for future high-resolution studies, and then rebinned again to a common linear wavelength binning of $0.5 \AA$ per flux point for more convenient use for working with colors and spectral indices. ${ }^{12}$

For each star in the grid of spectra described above, multiple realizations of the same spectrum were calculated with singleelement abundance adjustments, so that the effects of $\mathrm{C}, \mathrm{N}$, $\mathrm{O}, \mathrm{Ne}, \mathrm{Mg}, \mathrm{Si}, \mathrm{S}, \mathrm{Ca}, \mathrm{Ti}$, and $\mathrm{Fe}$ could be individually gauged. For neon enhancement, we use the scaled-solar spectra because of the small effect on the optical stellar spectrum from neon. Any isochrone-level temperature, luminosity, or stellar lifetime effects that we depicted in Paper I from neon are, however, included. Thus, at solar metallicity, 35 gravity and temperature combinations $\times 10$ element adjustments (counting the nonadjusted solar abundance spectrum) makes a total of 350 spectra.

Another convenience is the definition of $\mathrm{R}$, which stands for "generic heavy element." Usually, one refers to relative abundance changes relative to iron, such as $[\mathrm{O} / \mathrm{Fe}]$ or $[\mathrm{Mg} / \mathrm{Fe}]$. However, this stops making sense in the case of wanting to

12 We have used synthetic stellar spectra with an elemental enhancement by 0.3 dex in this study. As discussed in Tripicco \& Bell (1995), however, a 0.15 dex enhancement is used for carbon in order to avoid the carbon star abundance regime where carbon atoms outnumber oxygen atoms. 
Table 3

Color Behavior with Enhancements at Constant $[\mathrm{R} / \mathrm{H}]$

\begin{tabular}{|c|c|c|c|c|c|c|c|c|c|c|c|}
\hline Color & $\mathrm{C}$ & $\mathrm{N}$ & $\mathrm{O}$ & $\mathrm{Ne}$ & $\mathrm{Mg}$ & $\mathrm{Si}$ & $\mathrm{S}$ & $\mathrm{Ca}$ & $\mathrm{Ti}$ & $\mathrm{Fe}$ & $\alpha$ \\
\hline \multicolumn{12}{|c|}{ Dwarf, $T_{\text {eff }}=4000 \mathrm{~K}$} \\
\hline$U-B$ & -11 & 10 & 30 & $\cdots$ & -65 & -35 & 0 & -40 & 15 & 139 & -97 \\
\hline$B-V$ & 22 & 0 & -31 & $\ldots$ & -17 & 38 & 0 & -7 & 15 & 26 & -5 \\
\hline$V-R$ & 0 & 0 & 0 & $\ldots$ & 31 & 0 & 0 & -13 & 4 & 14 & 19 \\
\hline$V-I$ & -3 & 0 & 1 & $\ldots$ & 32 & -1 & 0 & -14 & 4 & 16 & 19 \\
\hline \multicolumn{12}{|c|}{ Dwarf, $T_{\text {eff }}=5000 \mathrm{~K}$} \\
\hline$U-B$ & 28 & 26 & -10 & $\ldots$ & -20 & -33 & 0 & -3 & 11 & 74 & -57 \\
\hline$B-V$ & 24 & 4 & -14 & $\ldots$ & -30 & 8 & 0 & 6 & 7 & 24 & -23 \\
\hline$V-R$ & -1 & -3 & 2 & $\ldots$ & 9 & -1 & 0 & 0 & 3 & 12 & 11 \\
\hline$V-I$ & -10 & -10 & 7 & $\ldots$ & 11 & 0 & 0 & -1 & 3 & 16 & 18 \\
\hline \multicolumn{12}{|c|}{ Dwarf, $T_{\text {eff }}=6000 \mathrm{~K}$} \\
\hline$U-B$ & 8 & 17 & 1 & $\ldots$ & 11 & -13 & 0 & 2 & 8 & 41 & 8 \\
\hline$B-V$ & 13 & 3 & 0 & $\ldots$ & -12 & -5 & 0 & 7 & 5 & 15 & -8 \\
\hline$V-R$ & 2 & 0 & 0 & $\ldots$ & 0 & 0 & 0 & 0 & 2 & 8 & 0 \\
\hline$V-I$ & 1 & -2 & 0 & $\ldots$ & 0 & 0 & 0 & 0 & 2 & 11 & 0 \\
\hline \multicolumn{12}{|c|}{ Giant, $T_{\text {eff }}=4000 \mathrm{~K}$} \\
\hline$U-B$ & 21 & 18 & 1 & $\ldots$ & -20 & -44 & 0 & -20 & 22 & 125 & -63 \\
\hline$B-V$ & 22 & 0 & -27 & $\ldots$ & -28 & 39 & 0 & -3 & 12 & 23 & -10 \\
\hline$V-R$ & -9 & -4 & 5 & $\ldots$ & 21 & 0 & 0 & -8 & 3 & 13 & 19 \\
\hline$V-I$ & -22 & -9 & 11 & $\ldots$ & 23 & 0 & 0 & -9 & 2 & 17 & 26 \\
\hline \multicolumn{12}{|c|}{ Giant, $T_{\text {eff }}=5000 \mathrm{~K}$} \\
\hline$U-B$ & 29 & 33 & -9 & $\ldots$ & 31 & -32 & 0 & 7 & 11 & 34 & 5 \\
\hline$B-V$ & 24 & 8 & -9 & $\ldots$ & -19 & -3 & 0 & 8 & 6 & 13 & -20 \\
\hline$V-R$ & 4 & -9 & 0 & $\ldots$ & 2 & 0 & 0 & 0 & 2 & 11 & 5 \\
\hline$V-I$ & -6 & -22 & 6 & $\ldots$ & 7 & 2 & 0 & 0 & 3 & 17 & 17 \\
\hline \multicolumn{12}{|c|}{ Giant, $T_{\text {eff }}=6000 \mathrm{~K}$} \\
\hline$U-B$ & 3 & 11 & 1 & $\ldots$ & 16 & -5 & 0 & 7 & 8 & 17 & 25 \\
\hline$B-V$ & 7 & 2 & 0 & $\cdots$ & -3 & 0 & 0 & 10 & 5 & 14 & 9 \\
\hline$V-R$ & 1 & 0 & 0 & $\ldots$ & 0 & 0 & 0 & 0 & 2 & 6 & 0 \\
\hline$V-I$ & 1 & 0 & 0 & $\ldots$ & 0 & 0 & 0 & 0 & 2 & 8 & 0 \\
\hline
\end{tabular}

Note. 1 . All elements scaled individually by +0.3 dex, except $C$, which is increased by +0.15 dex.

Table 4

Model Population Color Behavior with Enhancements at Constant $Z$

\begin{tabular}{|c|c|c|c|c|c|c|c|c|c|c|c|c|}
\hline Color & $\begin{array}{c}\text { Synthetic } \\
\text { Color } \\
(\mathrm{mag})\end{array}$ & $\begin{array}{c}\Delta I \\
(\mathrm{C}) \\
(\mathrm{mmag})\end{array}$ & $\begin{array}{c}\Delta I \\
(\mathrm{~N}) \\
(\mathrm{mmag})\end{array}$ & $\begin{array}{c}\Delta I \\
(\mathrm{O}) \\
(\mathrm{mmag})\end{array}$ & $\begin{array}{c}\Delta I \\
(\mathrm{Ne}) \\
(\mathrm{mmag})\end{array}$ & $\begin{array}{c}\Delta I \\
(\mathrm{Mg}) \\
(\mathrm{mmag})\end{array}$ & $\begin{array}{c}\Delta \\
(\mathrm{Si}) \\
(\mathrm{mmag})\end{array}$ & $\begin{array}{c}\Delta I \\
(\mathrm{~S}) \\
(\mathrm{mmag})\end{array}$ & $\begin{array}{c}\Delta I \\
(\mathrm{Ca}) \\
(\mathrm{mmag})\end{array}$ & $\begin{array}{c}\Delta I \\
(\mathrm{Ti}) \\
(\mathrm{mmag})\end{array}$ & $\begin{array}{c}\Delta I \\
(\mathrm{Fe}) \\
(\mathrm{mmag})\end{array}$ & $\begin{array}{c}I \\
(\alpha) \\
(\mathrm{mmag})\end{array}$ \\
\hline \multicolumn{13}{|c|}{ Population age $1 \mathrm{Gyr}$} \\
\hline$B C_{V}$ & $\cdots$ & -0.3 & 0.0 & 5.5 & 1.9 & 2.9 & 2.6 & 1.6 & -1.2 & 0.8 & -3.8 & 8.0 \\
\hline$U-B$ & 0.050 & -3.7 & 2.3 & -14.9 & -4.0 & 1.6 & -5.5 & -1.9 & 2.6 & 2.2 & 14.8 & -20.7 \\
\hline$B-V$ & 0.361 & -1.0 & -0.2 & -34.6 & 1.3 & 5.7 & 9.1 & 6.2 & 6.0 & 2.4 & -1.1 & -11.6 \\
\hline$V-R$ & 0.223 & -1.1 & -0.2 & -17.1 & 2.4 & 7.6 & 7.4 & 4.8 & 1.0 & 1.9 & -1.7 & -2.3 \\
\hline$V-I$ & 0.458 & -4.0 & 0.4 & -26.3 & 5.8 & 15.2 & 15.2 & 10.7 & 2.0 & 7.2 & -7.6 & 4.5 \\
\hline \multicolumn{13}{|c|}{ Population age $2 \mathrm{Gyr}$} \\
\hline$B C_{V}$ & $\ldots$ & -9.9 & -4.5 & 11.1 & 0.7 & 12.2 & 4.5 & 0.6 & -17.5 & 15.5 & 3.9 & 46.6 \\
\hline$U-B$ & 0.171 & 0.5 & 12.1 & -30.2 & 4.9 & 7.7 & -6.6 & 4.8 & -2.8 & 16.7 & 12.5 & -18.4 \\
\hline$B-V$ & 0.664 & 16.1 & 6.3 & -20.6 & 12.9 & -10.0 & 10.4 & 8.2 & -2.4 & 15.2 & -2.5 & -10.4 \\
\hline$V-R$ & 0.428 & 0.5 & -1.6 & -1.9 & 6.6 & 8.8 & 7.1 & 4.2 & -14.2 & 14.2 & 4.6 & 22.0 \\
\hline$V-I$ & 0.877 & -15.5 & -4.9 & 10.0 & 8.5 & 23.3 & 13.9 & 6.2 & -43.3 & 47.2 & 9.8 & 97.5 \\
\hline \multicolumn{13}{|c|}{ Populatio } \\
\hline$B C_{V}$ & $\ldots$ & -21.1 & -11.1 & 10.5 & -2.8 & 12.0 & 0.0 & -3.0 & -2.7 & 20.2 & -1.6 & 48.8 \\
\hline$U-B$ & 0.369 & -10.7 & 5.7 & -75.5 & -13.1 & 14.9 & -19.8 & -8.1 & -2.7 & 5.1 & 41.1 & -71.3 \\
\hline$B-V$ & 0.861 & 9.7 & -4.8 & -52.3 & -4.3 & -15.6 & 5.8 & -5.7 & 1.5 & -3.0 & 15.1 & -54.8 \\
\hline$V-R$ & 0.537 & -9.1 & -10.8 & -15.6 & -3.3 & 11.2 & 1.4 & -3.7 & -3.9 & 6.2 & 8.7 & 4.2 \\
\hline$V-I$ & 1.087 & -40.9 & -22.5 & -3.5 & -8.1 & 18.4 & -0.4 & -8.4 & -5.7 & 40.6 & 5.0 & 71.9 \\
\hline \multicolumn{13}{|c|}{ Population age $12 \mathrm{Gyr}$} \\
\hline$B C_{V}$ & $\ldots$ & -22.5 & -10.4 & 11.8 & -3.5 & 21.4 & 2.8 & -1.5 & -1.2 & 30.4 & 7.2 & 55.3 \\
\hline$U-B$ & 0.599 & 2.5 & 14.7 & -101.8 & -15.7 & 31.1 & -14.7 & -7.1 & -0.8 & 9.2 & 68.8 & -99.0 \\
\hline$B-V$ & 0.988 & 21.1 & 0.8 & -58.1 & -4.9 & -8.3 & 15.7 & -3.4 & 4.2 & -0.4 & 26.3 & -65.8 \\
\hline$V-R$ & 0.604 & -6.1 & -8.4 & -15.0 & -3.6 & 22.4 & 5.3 & -1.8 & -2.3 & 11.7 & 17.1 & 5.8 \\
\hline$V-I$ & 1.200 & -37.2 & -17.3 & 1.5 & -8.4 & 36.3 & 7.9 & -3.9 & -1.6 & 57.2 & 22.2 & 78.6 \\
\hline
\end{tabular}

Note. 1. All elements scaled individually by +0.3 dex, except $C$, which is increased by +0.2 dex (see footnote 18 ). 


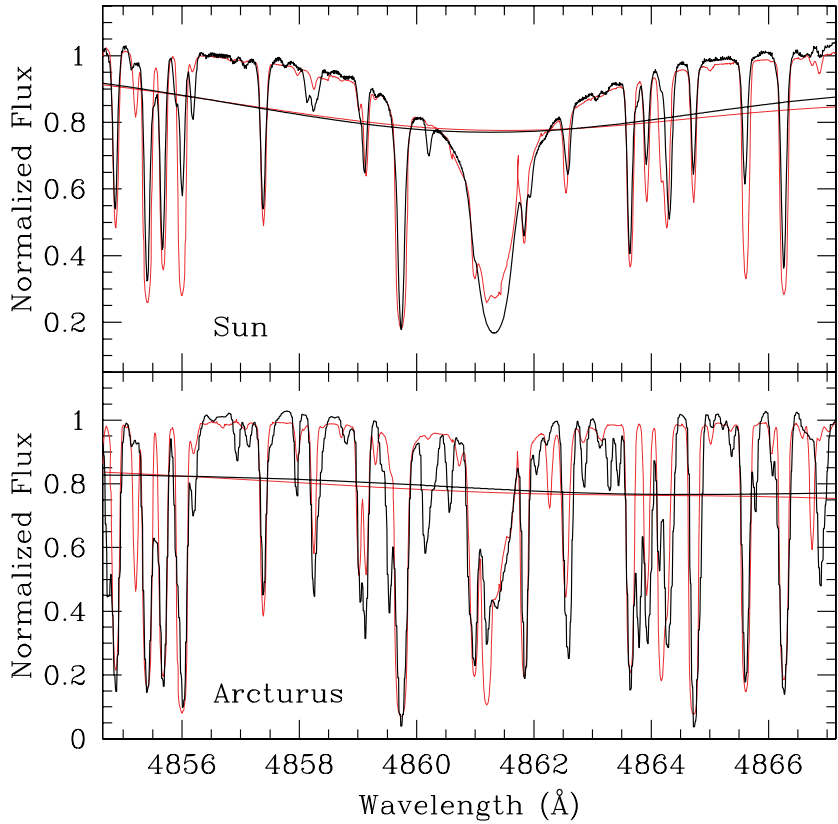

Figure 1. Synthetic and observed spectra are compared near the region of the $\mathrm{H} \beta$ lines. The top panel is the solar spectra and the bottom panel is Arcturus one. The thicker (black) lines are the observations. The degraded low-resolution spectra at $200 \mathrm{~km} \mathrm{~s}^{-1}$ are overlaid on top of the high-resolution spectra. It is seen that the notable mismatches between the synthetic and the observed spectra at the high-resolution comparisons are smeared out at the low resolution.

(A color version of this figure is available in the online journal.)

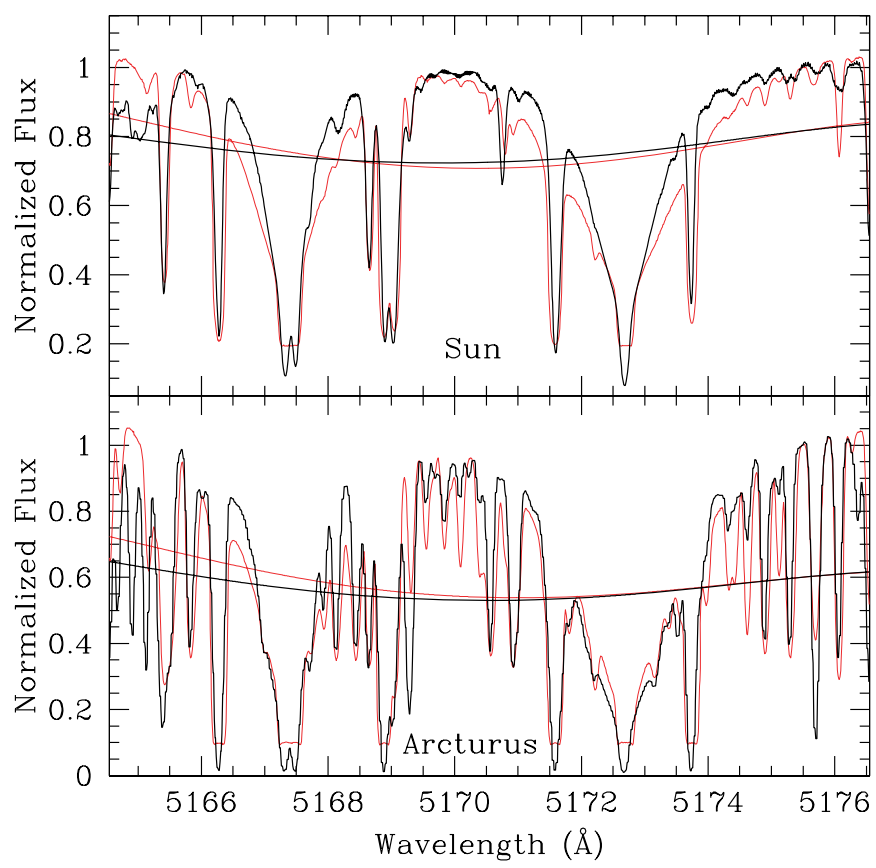

Figure 2. Same as Figure 1, but near the region of the $\mathrm{Mg} b$ lines. (A color version of this figure is available in the online journal.)

change Fe with respect to other elements, i.e., using $[\mathrm{Fe} / \mathrm{Fe}]$ leads to nonsense. So we use the "generic heavy element" R to stand for "all heavy elements that remain scaled with the solar mixture, where all other element tweaks must be specified." With that notation, $[\mathrm{Fe} / \mathrm{R}]$ is perfectly acceptable, and the specification of constant $[\mathrm{R} / \mathrm{H}]$ means that all elements except

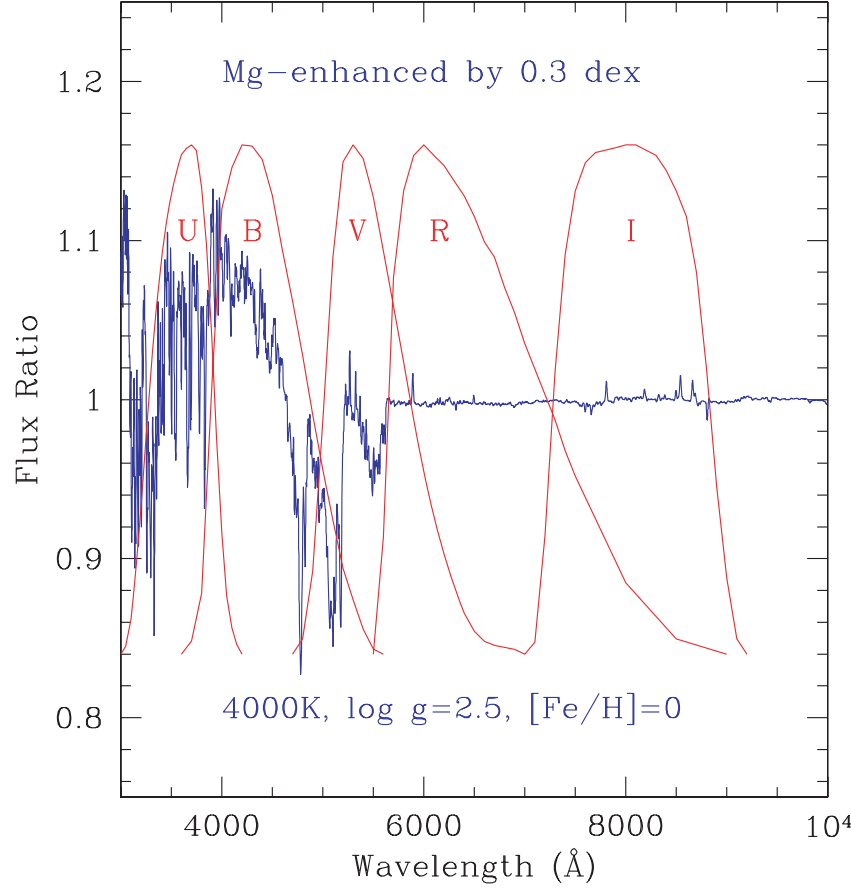

Figure 3. At $4000 \mathrm{~K}$ and $\log g=2.5$, solar-scaled and Mg-enhanced spectra are divided. $U B V R I$-band filter response curves are overlaid. The blue colors of $U-B$ and $B-V$ and the red colors of $V-R$ and $V-I$ from the $\mathrm{Mg}$-enhanced spectra that we read in Table 3 are visually elucidated here.

(A color version of this figure is available in the online journal.)

the one (or, more generically, ones) under consideration are held in solar lockstep.

\subsection{Synthetic Spectra Accuracy}

An interpolation routine was constructed so that spectra of arbitrary temperature, gravity, and abundance mixture could be produced on demand. Linear interpolation in the log of the flux seemed to give the most predictive results. Interpolation was done for all input variables: temperature, $\log g$, and the whole set of abundance parameters. Tests in which one star in the grid was compared with its interpolated version calculated from the flanking temperature grid points indicate that, within the dense $4000-6000 \mathrm{~K}$ part of the grid, interpolations yield better than $1 \%$ accuracy at any given flux point, since the spectra change more with temperature than with the other parameters. Given the nonlinear temperature response of many lines, e.g., Gray \& Brown (2001), this is encouragingly good. Doubtless, in the future, we will demand a denser temperature grid, but $1 \%$ accuracy is better than we need for the present study.

Toward the more fundamental question of how well the synthetic spectra match real spectra, there is little we can add to discussions in Martins \& Coelho (2007), Coelho et al. (2005), Korn et al. (2005), and Tripicco \& Bell (1995). Serven et al. (2005) did discover some too-strong lines due to chromium (that is, mistakes in the line list) via stellar comparisons, but these lines do not affect the present conclusions at all. More worrisome is silicon because the features that show $\mathrm{Si}$ effects are mostly $\mathrm{SiH}$ lines in the blue, but these line lists may be immature (R. Peterson 2007, private communication). All conclusions regarding $\mathrm{Si}$ should be regarded with great suspicion until this question is satisfactorily resolved.

In Figures 1 and 2, we compare the high-resolution spectra of the Sun and Arcturus with the grid-interpolated synthetic spectra 
Table 5

Model Population Index Behavior with Element Enhancements

\begin{tabular}{|c|c|c|c|c|c|c|c|c|c|c|c|c|c|c|c|}
\hline $\begin{array}{l}\text { Index } \\
\text { Name } \\
\end{array}$ & $\begin{array}{l}\text { Index } \\
\text { Value } \\
\end{array}$ & $\begin{array}{l}\Delta I \\
(\mathrm{C}) \\
\end{array}$ & $\begin{array}{c}\Delta I \\
(\mathrm{C}+) \\
\end{array}$ & $\begin{array}{l}\Delta I \\
(\mathrm{~N}) \\
\end{array}$ & $\begin{array}{c}\Delta I \\
(\mathrm{~N}+) \\
\end{array}$ & $\begin{array}{l}\Delta I \\
(\mathrm{O}) \\
\end{array}$ & $\begin{array}{c}\Delta I \\
(\mathrm{O}+)\end{array}$ & $\begin{array}{c}\Delta I \\
(\mathrm{Mg}) \\
\end{array}$ & $\begin{array}{l}\Delta I \\
(\mathrm{Si}) \\
\end{array}$ & $\begin{array}{l}\Delta I \\
(\mathrm{~S}) \\
\end{array}$ & $\begin{array}{c}\Delta I \\
(\mathrm{Ca}) \\
\end{array}$ & $\begin{array}{c}\Delta I \\
(\mathrm{Ti}) \\
\end{array}$ & $\begin{array}{c}\Delta I \\
(\mathrm{Fe})\end{array}$ & $\begin{array}{l}\Delta I \\
(\alpha) \\
\end{array}$ & $\begin{array}{c}\Delta I \\
(\alpha+) \\
\end{array}$ \\
\hline \multicolumn{16}{|c|}{ Population age $1 \mathrm{Gyr}$, isochrone effects only } \\
\hline $\mathrm{CN}_{1}$ & -0.1729 & -0.0013 & 0.0052 & -0.0003 & 0.0015 & -0.0091 & 0.0096 & 0.0029 & 0.0027 & 0.0014 & 0.0004 & -0.0001 & 0.0037 & -0.0030 & 0.0317 \\
\hline Ca4227 & 0.3702 & -0.0054 & 0.0184 & -0.0032 & 0.0029 & -0.0492 & 0.0247 & 0.0100 & 0.0098 & 0.0061 & 0.0018 & -0.0003 & & -0.0 & 0.1102 \\
\hline G4300 & -0.1319 & -0.0663 & 0.2024 & -0.0232 & 0.05 & -0.40 & 0.3611 & 0.1018 & 0.0976 & 0.0473 & 05 & -0.0048 & & -0.2 & 1.1606 \\
\hline Fe4383 & & -0.0418 & 0.0 & -0.0 & 0.0337 & -0.27 & 0.1782 & 0.0566 & 0.0289 & 0.0066 & 0.0178 & 0.0081 & 0.2371 & -0.2220 & 0.7064 \\
\hline $\mathrm{C}_{2} 4668$ & 0.3137 & -0.0962 & 0.0786 & -0.0405 & 0.0117 & -0.4479 & 0.1322 & 0.0245 & 0.0041 & 0.0059 & 0.0118 & -0.0001 & 0.7050 & -0.4688 & 0.5675 \\
\hline $\mathrm{H} \beta$ & 6.0151 & -0.0298 & -0.1859 & -0.0043 & -0.0386 & 0.1792 & -0.2727 & -0.1091 & -0.1003 & -0.0425 & -0.0073 & 0.0115 & 0.0807 & -0.0603 & -1.0362 \\
\hline Fe5015 & 2.4643 & -0.0762 & 0.0633 & -0.0264 & 0.0157 & -0.3389 & 0.1203 & 0.0219 & 0.0105 & 0.0090 & 0.0116 & 0.0009 & 0.4 & -0.3421 & 0.4301 \\
\hline $\mathrm{Mg}_{1}$ & 0.0013 & 0.0006 & 0.0004 & 0.0006 & -0.0001 & 0.0003 & 0.0000 & 0.0011 & 0.0006 & 0.0004 & 0.0006 & 0.0002 & -0.0 & -0.0001 & 0.0059 \\
\hline $\mathrm{Mg}_{2}$ & 0.0578 & -0.0001 & 0.0017 & 0.0006 & 0.0001 & -0.0034 & 0.0018 & 0.0022 & 0.0013 & 0.0009 & 0.0010 & 0.0005 & 0.0023 & -0.0034 & 0.0140 \\
\hline $\operatorname{Mg} b$ & 0.9845 & -0.0053 & 0.0357 & 0.0078 & 0.0074 & -0.0440 & 0.0625 & 0.0421 & 0.0296 & 0.0197 & 0.0147 & 0.0091 & -0.0036 & -0.0129 & 0.2977 \\
\hline $\mathrm{NaD}$ & 1.1216 & 0.0455 & 0.0516 & 0.0090 & -0.0037 & -0.0638 & -0.0174 & 0.0305 & 0.0231 & 0.0132 & 0.0051 & -0.0049 & 0.0294 & -0.0684 & 0.1801 \\
\hline $\mathrm{TiO}_{1}$ & 0.0114 & 0.0034 & 0.0020 & -0.0008 & -0.0011 & -0.0010 & -0.0035 & -0.0001 & -0.0001 & -0.0009 & -0.0015 & -0.0013 & 0.0004 & 0.0000 & -0.0005 \\
\hline $\mathrm{TiO}_{2}$ & -0.0075 & 0.0079 & 0.0046 & -0.0020 & -0.0026 & -0.0026 & -0.0080 & -0.0001 & -0.0001 & -0.0021 & -0.0032 & -0.0031 & 0.0006 & -0.0001 & -0.0004 \\
\hline $\mathrm{H} \delta_{A}$ & 8.7930 & 0.0021 & -0.3210 & -0.0001 & -0.0908 & 0.3505 & -0.5622 & -0.1823 & -0.1727 & -0.0756 & -0.0182 & 0.0092 & 0.1019 & -0.0687 & -2.0267 \\
\hline $\mathrm{H} \gamma_{A}$ & 9.0232 & -0.0102 & -0.4023 & -0.0039 & -0.1157 & 0.4442 & -0.6934 & -0.2312 & -0.2128 & -0.0889 & -0.0256 & 0.0143 & 0.1671 & -0.1038 & -2.6081 \\
\hline $\mathrm{H} \delta_{F}$ & 5.8139 & 0.0277 & -0.2032 & 0.0104 & -0.0573 & 0.2863 & -0.3554 & -0.10 & -0.1054 & -0.0484 & -0.0079 & 0.0064 & -0.0646 & 0.0497 & -1.1933 \\
\hline $\mathrm{H} \gamma_{F}$ & 6.1194 & 0.0129 & -0.2280 & 0.0024 & -0.0605 & 0.2801 & -0.3875 & -0.1305 & -0.1233 & -0.0575 & -0.0135 & 0.0062 & -0.0013 & 0.0067 & -1.3902 \\
\hline
\end{tabular}

Population age $1 \mathrm{Gyr}$, isochrone plus spectral effects

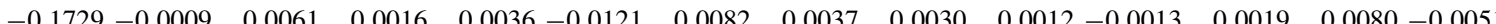

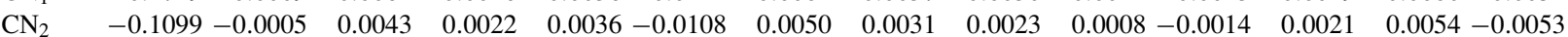
$\begin{array}{llllllllllllll}\mathrm{Ca} 4227 & 0.3702 & -0.0349 & 0.0020 & -0.0190 & -0.0060-0.0895 & 0.0319 & 0.0048 & 0.0035 & 0.0034 & 0.1021 & 0.0050 & 0.0892 & 0.0048\end{array}$ $\begin{array}{llllllllllllll}\text { G4300 } & -0.1319 & 0.0953 & 0.4853-0.0681 & 0.0570-0.7360 & 0.3229 & 0.0262 & 0.0345 & 0.0270 & 0.0934 & 0.1973 & 0.2422 & -0.4542\end{array}$ $\begin{array}{llllllllllll}\mathrm{Fe} 4383 & 0.0769-0.0556 & 0.1236-0.0304 & 0.0319-0.4444 & 0.1498 & 0.0248-0.0315-0.0029-0.0478 & 0.1234 & 0.4107-0.4621\end{array}$ $\begin{array}{lllllllllllll}\mathrm{Ca} 4455 & 0.4773-0.0417 & 0.0275-0.0196 & 0.0059-0.1871 & 0.0428-0.0278-0.0350-0.0003 & 0.0162 & 0.0251 & 0.0500-0.2267\end{array}$ $\begin{array}{llllllllllllll}\text { Fe4531 } & 1.4918-0.0932 & 0.0524-0.0416 & 0.0130-0.4076 & 0.0831 & -0.0291 & -0.0344-0.0025 & 0.0058 & 0.2521 & 0.1914 & -0.2945\end{array}$

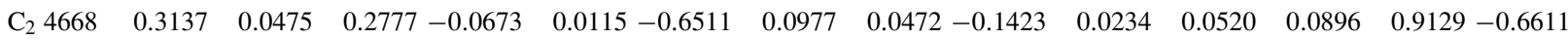
$\begin{array}{llllllllllllll}\mathrm{H} \beta & 6.0151-0.0295-0.1969 & 0.0020 & -0.0367 & 0.2201 & -0.2554 & -0.1288 & -0.0934 & -0.0403 & -0.0094 & 0.0493 & -0.0273 & 0.0027\end{array}$ $\begin{array}{lllllllllllllll}\text { Fe5015 } & 2.4643-0.1859 & 0.0965-0.0993 & 0.0160-0.8476 & 0.1272 & -0.0770-0.1042-0.0227 & 0.0789 & 0.2735 & 0.9007 & -0.8013\end{array}$ $\begin{array}{llllllllllll}\mathrm{Mg}_{1} & 0.0013 & 0.0021 & 0.0032 & -0.0001 & -0.0002-0.0044-0.0008 & 0.0048-0.0011 & 0.0002 & 0.0001 & 0.0008 & 0.0001 & -0.0036\end{array}$ $\begin{array}{llllllllllll}\mathrm{Mg}_{2} & 0.0578-0.0008 & 0.0031-0.0006 & 0.0001-0.0112 & 0.0014 & 0.0163-0.0017 & 0.0004 & 0.0010 & 0.0021 & 0.0034 & -0.0017\end{array}$ $\begin{array}{llllllllllll}\mathrm{Mg} b & 0.9845-0.0484 & 0.0239-0.0079 & 0.0078-0.1458 & 0.0643 & 0.4731-0.0047 & 0.0120 & 0.0248 & 0.0143 & -0.0420 & 0.2288\end{array}$ $\begin{array}{llllllllllllll}\text { Fe5270 } & 1.0894-0.1036 & 0.0349-0.0354 & 0.0133-0.4167 & 0.0637 & -0.0172 & 0.1169 & 0.0096 & 0.1063 & 0.0534 & 0.3233 & -0.2704\end{array}$ $\begin{array}{llllllllllll}\text { Fe5335 } & 0.8774-0.1122 & 0.0176-0.0350 & 0.0094-0.3570 & 0.0868-0.0184 & 0.1227 & -0.0009 & 0.0030 & 0.0324 & 0.3424 & -0.3239\end{array}$ $\begin{array}{lllllllllllll}\text { Fe5406 } & 0.3673-0.0620 & 0.0008-0.0134 & 0.0050-0.1852 & 0.0360 & 0.0022 & 0.0242 & 0.0021 & 0.0072 & -0.0149 & 0.3128 & -0.2230\end{array}$ $\begin{array}{llllllllllll}\text { Fe5709 } & 0.4454-0.0470 & 0.0006-0.0130 & 0.0033-0.1317 & 0.0296 & 0.0218-0.0033 & 0.0153 & 0.0092 & 0.0142 & 0.1678-0.1249\end{array}$ $\begin{array}{llllllllllll}\text { Fe5782 } & 0.2933-0.0271 & 0.0009-0.0058 & 0.0010-0.0829 & 0.0138 & 0.0198-0.0051 & 0.0002 & 0.0046 & 0.0005 & 0.0177 & -0.0967\end{array}$

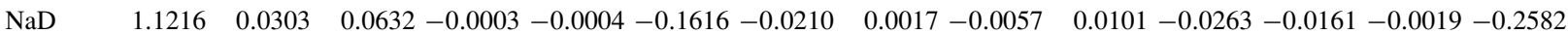
$\begin{array}{llllllllllll}\mathrm{TiO}_{1} & 0.0114 & 0.0028 & 0.0018 & -0.0008 & -0.0008 & -0.0023-0.0030 & -0.0003 & 0.0004-0.0010-0.0016 & 0.0008 & 0.0006 & 0.0004\end{array}$ $\mathrm{TiO}_{2} \quad-0.0075 \quad 0.0071 \quad 0.0038-0.0020-0.0025-0.0033-0.0072-0.0003-0.0004-0.0024-0.0043-0.0001 \quad 0.0014-0.0001$ $\begin{array}{lllllllllllll}\mathrm{H} \delta_{A} & 8.7930 & 0.0011-0.3916 & 0.0191-0.1020 & 0.5938 & -0.5279 & -0.1339-0.1226-0.0616 & 0.0439 & -0.0549 & -0.2367 & 0.2959\end{array}$ $\begin{array}{llllllll}\mathrm{H} \gamma_{A} & 9.0232-0.1151-0.6314 & 0.0461-0.1130 & 0.8066-0.6397-0.1076-0.1489-0.0668-0.0552-0.1770-0.0191 & 0.2940\end{array}$

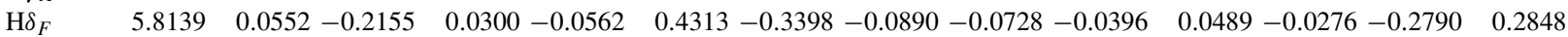
$\begin{array}{llllllll}\mathrm{H} \gamma_{F} & 6.1194-0.0558-0.3441 & 0.0210-0.0584 & 0.4152-0.3578-0.0815-0.0958-0.0493-0.0315 & -0.0107-0.0773 \quad 0.2051\end{array}$

0.0322 0.0225 0.3323 1.3367 0.5671 0.1421 0.5801 0.5911 $-0.9932$ 0.8161 0.0106 0.0394 1.0441 0.6047 0.4283 0.1452 0.1635 0.0828 0.0620 0.0040 0.0029 $-1.8286$ $-2.6081$ $-1.0708$ $-1.2780$

Population age $2 \mathrm{Gyr}$, isochrone effects only

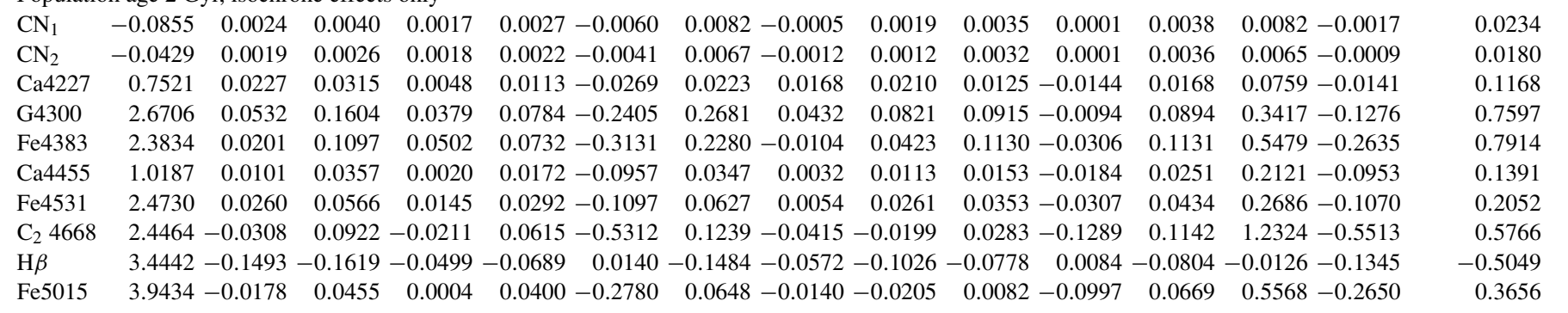


Table 5

Continued

\begin{tabular}{|c|c|c|c|c|c|c|c|c|c|c|c|c|c|c|c|}
\hline $\begin{array}{l}\text { Index } \\
\text { Name }\end{array}$ & $\begin{array}{l}\text { Index } \\
\text { Value }\end{array}$ & $\begin{array}{l}\Delta I \\
(\mathrm{C})\end{array}$ & $\begin{array}{c}\Delta I \\
(\mathrm{C}+)\end{array}$ & $\begin{array}{l}\Delta I \\
(\mathrm{~N})\end{array}$ & $\begin{array}{c}\Delta I \\
(\mathrm{~N}+)\end{array}$ & $\begin{array}{l}\Delta I \\
(\mathrm{O})\end{array}$ & $\begin{array}{c}\Delta I \\
(\mathrm{O}+)\end{array}$ & $\begin{array}{c}\Delta I \\
(\mathrm{Mg})\end{array}$ & $\begin{array}{c}\Delta I \\
(\mathrm{Si})\end{array}$ & $\begin{array}{l}\Delta I \\
(\mathrm{~S})\end{array}$ & $\begin{array}{c}\Delta I \\
(\mathrm{Ca})\end{array}$ & $\begin{array}{c}\Delta I \\
(\mathrm{Ti})\end{array}$ & $\begin{array}{c}\Delta I \\
(\mathrm{Fe})\end{array}$ & $\begin{array}{l}\Delta I \\
(\alpha)\end{array}$ & $\begin{array}{c}\Delta I \\
(\alpha+)\end{array}$ \\
\hline $\mathrm{Mg}_{1}$ & 0.0439 & 0.0030 & 0.0032 & 0.0016 & 0.0015 & -0.0010 & 0.0029 & 0.0005 & 0.0023 & 0.0026 & -0.0012 & 0.0027 & 0.0106 & -0.0015 & 0.0077 \\
\hline $\mathrm{Mg}_{2}$ & 0.1326 & 0.0039 & 0.0050 & 0.0018 & 0.0021 & -0.0049 & 0.0048 & 0.0014 & 0.0025 & 0.0029 & -0.0048 & 0.0043 & 0.0232 & -0.0045 & 0.0212 \\
\hline $\operatorname{Mg} b$ & 2.0402 & 0.0364 & 0.0486 & 0.0154 & 0.0221 & -0.0648 & 0.0711 & 0.0351 & 0.0275 & 0.0328 & -0.0648 & 0.0538 & 0.2308 & -0.0252 & 0.3892 \\
\hline Fe5270 & 2.0614 & -0.0059 & 0.0285 & 0.0194 & 0.0261 & -0.1232 & 0.0658 & -0.0104 & 0.0078 & 0.0389 & -0.0319 & 0.0447 & 0.2649 & -0.1341 & 0.2021 \\
\hline Fe5335 & 1.8585 & -0.0153 & 0.0322 & 0.0140 & 0.0261 & -0.1486 & 0.0678 & -0.0079 & 0.0089 & 0.0359 & -0.0388 & 0.0442 & 0.3550 & -0.1747 & 0.1918 \\
\hline Fe5406 & 1.0693 & 0.0062 & 0.0258 & 0.0140 & 0.0196 & -0.0807 & 0.0499 & -0.0136 & 0.0091 & 0.0309 & -0.0168 & 0.0333 & 0.1989 & -0.0935 & 0.1176 \\
\hline Fe5709 & 0.7623 & -0.0270 & -0.0089 & 0.0082 & 0.0066 & -0.0558 & 0.0446 & -0.0249 & -0.0058 & 0.0220 & 0.0168 & 0.0140 & 0.0845 & -0.0766 & 0.0474 \\
\hline Fe5782 & 0.6065 & -0.0069 & 0.0004 & 0.0095 & 0.0079 & -0.0326 & 0.0265 & -0.0167 & -0.0005 & 0.0182 & -0.0059 & 0.0162 & 0.0971 & -0.0482 & 0.0300 \\
\hline $\mathrm{NaD}$ & 2.1600 & 0.1099 & 0.1152 & 0.0102 & 0.0364 & -0.1702 & -0.0175 & 0.0072 & 0.0417 & 0.0317 & -0.0799 & 0.0453 & 0.5593 & -0.2156 & 0.0953 \\
\hline $\mathrm{TiO}_{1}$ & 0.0261 & 0.0075 & 0.0050 & -0.0014 & 0.0007 & -0.0003 & -0.0061 & 0.0029 & 0.0014 & -0.0030 & -0.0085 & 0.0010 & 0.0184 & 0.0031 & 0.0000 \\
\hline $\mathrm{TiO}_{2}$ & 0.0293 & 0.0177 & 0.0117 & -0.0031 & 0.0017 & -0.0013 & -0.0132 & 0.0058 & 0.0037 & -0.0058 & -0.0166 & 0.0021 & 0.0409 & 0.0055 & -0.0009 \\
\hline $\mathrm{H} \delta_{A}$ & 3.4142 & -0.1725 & -0.3488 & -0.1243 & -0.1800 & 0.2600 & -0.5152 & -0.1256 & -0.2074 & -0.2125 & 0.0263 & -0.1989 & -0.2548 & -0.0748 & -1.6128 \\
\hline $\mathrm{H} \gamma_{A}$ & 1.7203 & -0.2679 & -0.4682 & -0.1819 & -0.2445 & 0.3604 & -0.6567 & -0.1653 & -0.2646 & -0.2901 & 0.0721 & -0.2717 & -0.2918 & -0.1146 & -2.3564 \\
\hline $\mathrm{H} \delta_{F}$ & 2.8724 & -0.0923 & -0.1963 & -0.0585 & -0.0947 & 0.1466 & -0.2873 & -0.0819 & -0.1200 & -0.1046 & 0.0111 & -0.096 & -0.1627 & -0.0260 & -0.8223 \\
\hline $\mathrm{H} \gamma_{F}$ & 2.4631 & -0.1487 & -0.2424 & -0.0789 & -0.1168 & 0.1471 & -0.3364 & -0.1001 & -0.1562 & -0.1424 & 0.0165 & -0.1327 & -0.1709 & -0.0683 & -1.0344 \\
\hline
\end{tabular}

Population age $2 \mathrm{Gyr}$, isochrone and spectral effects

$\begin{array}{lllllllllllllll}\mathrm{CN}_{1} & -0.0855 & 0.0080 & 0.0114 & 0.0132 & 0.0154 & -0.0193 & 0.0021 & -0.0019 & 0.0046 & 0.0030 & -0.0033 & 0.0058 & 0.0085 & -0.0164\end{array}$

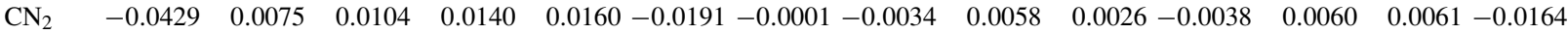

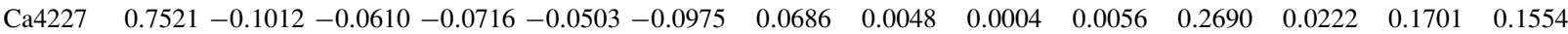
$\begin{array}{lllllllllllllll}\text { G4300 } & 2.6706 & 0.5505 & 0.7466 & 0.0031 & 0.0839 & -0.7120 & 0.1122 & -0.1588 & -0.0841 & 0.0722 & 0.0821 & 0.3310 & 0.1024 & -0.6526\end{array}$ $\begin{array}{lllllllllllllll}\mathrm{Fe} 4383 & 2.3834 & 0.0775 & 0.2567 & 0.0018 & 0.0693 & -0.6734 & 0.1963 & -0.2398 & -0.1479 & 0.0921 & -0.1526 & 0.2185 & 1.2160 & -1.0831\end{array}$ $\begin{array}{llllllllllllllll}\mathrm{C} a 4455 & 1.0187-0.0234 & 0.0310 & -0.0143 & 0.0158 & -0.2026 & 0.0384 & -0.0364 & -0.0265 & 0.0087 & 0.0091 & 0.0575 & 0.0398 & -0.2344\end{array}$

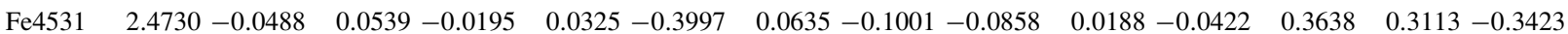

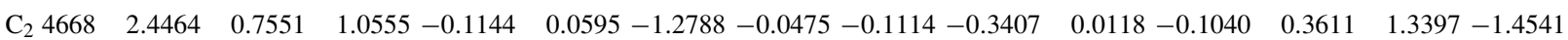
$\mathrm{H} \beta \quad 3.4442-0.1495-0.1757-0.0424-0.0680 \quad 0.0788-0.1291-0.1227-0.1021-0.0747 \quad 0.0093-0.0370-0.0788-0.0796$

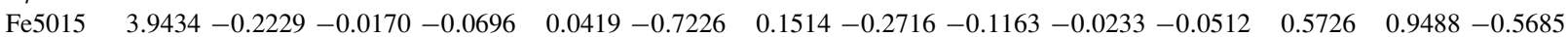
$\begin{array}{llllllllllllllll}\mathrm{Mg}_{1} & 0.0439 & 0.0150 & 0.0179 & 0.0001 & 0.0014 & -0.0162 & -0.0021 & 0.0168 & -0.0010 & 0.0021 & -0.0034 & 0.0014 & 0.0011 & -0.0077\end{array}$

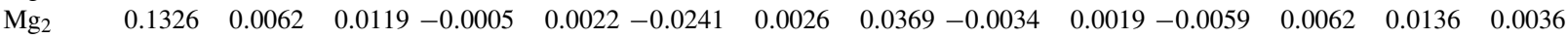
$\begin{array}{llllllllllllllll}\operatorname{Mg} b & 2.0402 & -0.0569 & 0.0150 & -0.0121 & 0.0243 & -0.2529 & 0.1000 & 0.9302 & -0.0697 & 0.0194 & -0.0693 & 0.0569 & 0.0326 & 0.5222\end{array}$ $\begin{array}{llllllllllllll}\text { Fe5270 } & 2.0614-0.0455 & 0.0662 & -0.0014 & 0.0451 & -0.4508 & 0.0351 & -0.1186 & 0.0032 & 0.0294 & 0.0706 & 0.0922 & 0.5833 & -0.4600\end{array}$ $\begin{array}{lllllllllllllll}\text { Fe5335 } & 1.8585 & -0.0857 & 0.0333 & -0.0231 & 0.0248 & -0.4247 & 0.0640 & -0.1098 & 0.0047 & 0.0201 & -0.0508 & 0.0682 & 0.7263 & -0.5722\end{array}$

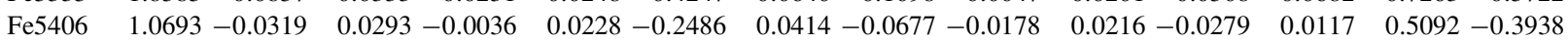
$\begin{array}{llllllllllll}\text { Fe5709 } & 0.7623-0.0553-0.0115 & -0.0133-0.0021-0.1575 & 0.0396 & -0.0153 & -0.0096 & 0.0220 & 0.0142 & 0.0348 & 0.1979 & -0.1642\end{array}$

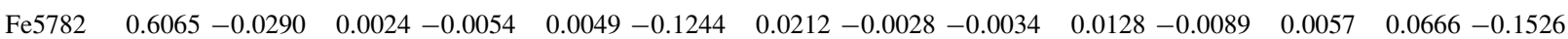

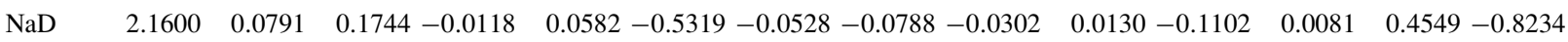
$\begin{array}{llllllllllllllll}\mathrm{TiO}_{1} & 0.0261 & 0.0033 & 0.0024 & -0.0011 & 0.0019 & 0.0003 & -0.0007 & 0.0028 & 0.0009 & -0.0033 & -0.0087 & 0.0087 & 0.0167 & 0.0103\end{array}$

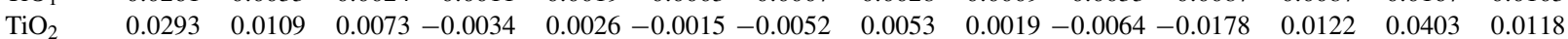
$\mathrm{H} \delta_{A} \quad 3.4142-0.2562-0.5249-0.1412-0.2482 \quad 0.6961-0.4302 \quad 0.1315-0.0090-0.1889 \quad 0.1551-0.2975-1.0744 \quad 0.8685$

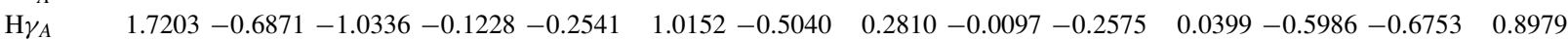
$\mathrm{H} \delta_{F} \quad 2.8724-0.0784-0.2105-0.0426-0.0950 \quad 0.2732-0.2815-0.0012 \quad 0.0166-0.0969 \quad 0.1023-0.1642-0.4546 \quad 0.3483$ $\mathrm{H} \gamma_{F} \quad 2.4631-0.4012-0.5422-0.0592-0.1180 \quad 0.3864-0.2484 \quad 0.0823-0.0491-0.1323-0.0062-0.1572-0.2800 \quad 0.4076$

0.0155 0.0117 0.6581 0.4429 0.1032 0.1708 0.3601 0.4946 $-0.5181$ 0.9104 0.0150 0.0664 1.7033 0.2179 0.0612 $-0.0389$ 0.0991 0.0400 $-0.2008$ 0.0193 0.0197 $-0.7611$ $-1.6287$ $-0.4492$ $-0.5306$

Population age $5 \mathrm{Gyr}$, isochrone effects only

$\begin{array}{llllllllll}\mathrm{CN}_{1} & -0.0160-0.0080 & 0.0015-0.0040 & 0.0006-0.0170 & 0.0057-0.0010 & 0.0000-0.0020-0.0010-0.0010 & 0.0250-0.0170\end{array}$ $\begin{array}{lllllllll}\mathrm{CN}_{2} & 0.0160-0.0090 & 0.0004-0.0040 & 0.0014-0.0160 & 0.0053-0.0010 & 0.0000-0.0020-0.0010-0.0010 & 0.0250-0.0160\end{array}$ $\begin{array}{llllllllll}\mathrm{Ca} 4227 & 1.1160-0.0030 & 0.0619-0.0170 & 0.0095-0.0720 & 0.0261 & 0.0500 & 0.0340-0.0060-0.0060-0.0040 & 0.0940-0.0700\end{array}$ $\begin{array}{llllllllll}\mathrm{G} 4300 & 4.6200-0.1490 & 0.1445-0.0610 & 0.0251-0.3510 & 0.2432 & 0.0590 & 0.0470-0.0380-0.0120-0.0130 & 0.3920-0.3100\end{array}$ $\begin{array}{lllllllllll}\mathrm{Fe} 4383 & 4.5220-0.3140 & 0.0489-0.1290 & 0.0059-0.6340 & 0.1811 & 0.0170 & 0.0090-0.0710-0.0210-0.0420 & 0.8690-0.7030\end{array}$ $\begin{array}{lllllllllll}\mathrm{C} 44455 & 1.4560-0.0370 & 0.0378-0.0290 & 0.0060-0.1600 & 0.0192 & 0.0180 & 0.0110-0.0130-0.0090-0.0090 & 0.2240-0.1840\end{array}$ $\begin{array}{lllllllllll}\mathrm{Fe} 4531 & 3.1680-0.0680 & 0.0393-0.0450 & 0.0030-0.2240 & 0.0203 & 0.0250 & 0.0160-0.0220-0.0140-0.0220 & 0.2740-0.2570\end{array}$ $\begin{array}{lllllll}\mathrm{C}_{2} 4668 & 4.1090-0.3150 & 0.0401-0.1610 & 0.0200-0.8720 & 0.0818-0.0310-0.0510-0.0910-0.0400-0.0270 & 1.4740-1.0680\end{array}$ $\begin{array}{lllllllllll}\mathrm{H} \beta & 2.2820-0.1130-0.1915 & 0.0040-0.0420 & 0.0820-0.0787 & -0.0940-0.0880-0.0090 & 0.0160 & -0.0060 & -0.0930 & 0.0170\end{array}$ Fe5015 $4.9640-0.1970-0.0111-0.0820-0.0064-0.4030 \quad 0.0322-0.0070-0.0290-0.0500-0.0200-0.0250 \quad 0.5470-0.4970$ $\begin{array}{llllllllll}\mathrm{Mg}_{1} & 0.0800-0.0030 & 0.0025-0.0030 & 0.0001-0.0110 & 0.0002 & 0.0030 & 0.0020-0.0020-0.0020 & -0.0020 & 0.0120-0.0120\end{array}$ $\begin{array}{llllllllll}\mathrm{Mg}_{2} & 0.2050-0.0080 & 0.0049-0.0050 & 0.0007-0.0200 & 0.0037 & 0.0050 & 0.0030-0.0020-0.0020-0.0020 & 0.0250-0.0220\end{array}$ $\begin{array}{lllllllll}\mathrm{Mg} b & 3.2300-0.1530 & 0.0614-0.0600 & 0.0025-0.2520 & 0.1154 & 0.0720 & 0.0380-0.0330-0.0070-0.0160 & 0.3280-0.2640\end{array}$ Fe5270 $2.7650-0.1510-0.0339-0.0550-0.0152-0.2320 \quad 0.0187-0.0080-0.0140-0.0310-0.0100-0.0310 \quad 0.2640-0.2780$ Fe5335 $2.5330-0.1630-0.0323-0.0640-0.0164-0.2680 \quad 0.0216-0.0020-0.0130-0.0360-0.0120-0.0330 \quad 0.3600-0.3270$ Fe5406 $1.5470-0.0980-0.0167-0.0440-0.0085-0.1850 \quad 0.0105-0.0040-0.0080-0.0250-0.0130-0.0240 \quad 0.2290-0.2210$ Fe5709 $0.9590-0.1070-0.0565-0.0280-0.0137-0.0920 \quad 0.0209-0.0280-0.0250-0.0200-0.0040-0.0160 \quad 0.1130-0.1180$ Fe5782 $0.7840-0.0690-0.0345-0.0230-0.0087-0.0790 \quad 0.0008-0.0160-0.0140-0.0160-0.0060-0.0150 \quad 0.0860-0.1020$ $\begin{array}{lllllllllllll}\mathrm{NaD} & 2.8340 & 0.1030 & 0.1704-0.0390 & 0.0247-0.3660-0.0616 & 0.0520 & 0.0520 & 0.0030-0.0230-0.0210 & 0.5570-0.4300\end{array}$ $\begin{array}{lllllllllllll}\mathrm{TiO}_{1} & 0.0240 & 0.0160 & 0.0131 & 0.0020 & 0.0043-0.0010-0.0036 & 0.0050 & 0.0050 & 0.0020-0.0010 & 0.0020 & 0.0060-0.0010\end{array}$

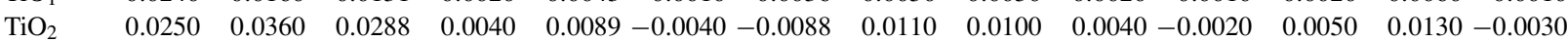

0.0123

0.0096

0.1238

0.4973

0.4978

0.0959

0.1394

0.2875

$-0.2373$

0.1363

0.0095

0.0199

0.3600

0.1220

0.1322

0.0842

0.0379

0.0194

0.0894

$-0.0038$ $-0.0086$ 
Table 5

Continued

\begin{tabular}{|c|c|c|c|c|c|c|c|c|c|c|c|c|c|c|c|}
\hline $\begin{array}{l}\text { Index } \\
\text { Name }\end{array}$ & $\begin{array}{l}\text { Index } \\
\text { Value }\end{array}$ & $\begin{array}{l}\Delta I \\
(\mathrm{C})\end{array}$ & $\begin{array}{c}\Delta I \\
(\mathrm{C}+)\end{array}$ & $\begin{array}{l}\Delta I \\
(\mathrm{~N})\end{array}$ & $\begin{array}{c}\Delta I \\
(\mathrm{~N}+)\end{array}$ & $\begin{array}{l}\Delta I \\
(\mathrm{O})\end{array}$ & $\begin{array}{c}\Delta I \\
(\mathrm{O}+)\end{array}$ & $\begin{array}{c}\Delta I \\
(\mathrm{Mg})\end{array}$ & $\begin{array}{l}\Delta I \\
(\mathrm{Si})\end{array}$ & $\begin{array}{l}\Delta I \\
(\mathrm{~S})\end{array}$ & $\begin{array}{c}\Delta I \\
(\mathrm{Ca})\end{array}$ & $\begin{array}{c}\Delta I \\
(\mathrm{Ti})\end{array}$ & $\begin{array}{c}\Delta I \\
(\mathrm{Fe})\end{array}$ & $\begin{array}{l}\Delta I \\
(\alpha)\end{array}$ & $\begin{array}{c}\Delta I \\
(\alpha+)\end{array}$ \\
\hline $\mathrm{H} \delta_{A}$ & -0.3970 & 0.1820 & -0.3275 & 0.1110 & -0.0640 & 0.6440 & -0.3895 & -0.1350 & -0.1160 & 0.0570 & 0.0340 & 0.0190 & -0.8510 & 0.5680 & -0.9208 \\
\hline $\mathrm{H} \gamma_{A}$ & -3.6950 & 0.1560 & -0.4981 & 0.1270 & -0.0870 & 0.7590 & -0.4980 & -0.2270 & -0.1880 & 0.0620 & 0.0450 & 0.0310 & -0.8820 & 0.6230 & -1.1746 \\
\hline $\mathrm{H} \delta_{F}$ & 0.9500 & 0.0750 & -0.1692 & 0.0430 & -0.0354 & 0.2540 & -0.2056 & -0.0810 & -0.0670 & 0.0230 & 0.0090 & 0.0040 & -0.2930 & 0.1900 & -0.4549 \\
\hline $\mathrm{H} \gamma_{F}$ & -0.2360 & 0.0820 & -0.2690 & 0.0620 & -0.0464 & 0.3570 & -0.2836 & -0.1350 & -0.1110 & 0.0290 & 0.0190 & 0.0140 & -0.4020 & 0.2630 & -0.6679 \\
\hline
\end{tabular}

Population age $5 \mathrm{Gyr}$, isochrone and spectral effects

$\begin{array}{lllllllllllll}\mathrm{CN}_{1} & -0.0160 & 0.0040 & 0.0187 & 0.0180 & 0.0260-0.0390-0.0063 & -0.0060 & 0.0070-0.0030-0.0060 & 0.0010 & 0.0190-0.0400\end{array}$

$\begin{array}{llllllllllll}\mathrm{CN}_{2} & 0.0160 & 0.0030 & 0.0187 & 0.0200 & 0.0287-0.0420-0.0084-0.0090 & 0.0120-0.0030-0.0070 & 0.0020 & 0.0180-0.0410\end{array}$

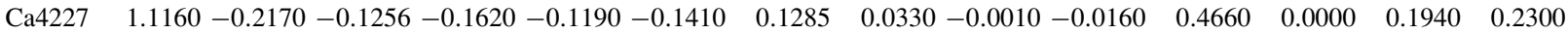

$\begin{array}{lllllll}\text { G4300 } & 4.6200 & 0.5190 & 0.8674-0.0710 & 0.0368-0.8280-0.0776-0.2190-0.1660-0.0490 & 0.1060 & 0.2570-0.0520-0.9350\end{array}$

Fe4383 $4.5220-0.2140 \quad 0.2685-0.1920-0.0011-1.1120 \quad 0.1418-0.4310-0.3010-0.0970-0.2120 \quad 0.0520 \quad 2.1490-1.9830$

$\begin{array}{lllllllllll}\text { Ca4455 } & 1.4560-0.0800 & 0.0303-0.0490 & 0.0037-0.2930 & 0.0269-0.0320-0.0260-0.0210 & 0.0310 & 0.0320 & 0.0020-0.3440\end{array}$

$\begin{array}{lllllll}\mathrm{Fe} 4531 & 3.1680-0.1420 & 0.0349-0.0760 & 0.0082-0.5120 & 0.0198-0.1300-0.1460-0.0380-0.0470 & 0.3320 & 0.3180-0.5500\end{array}$

$\begin{array}{lllllllll}\mathrm{C}_{2} 4668 & 4.1090 & 0.8430 & 1.5366-0.2990 & 0.0157-1.9260-0.1637-0.1800-0.5090-0.1330-0.0200 & 0.3330 & 1.5040-2.2890\end{array}$

$\mathrm{H} \beta \quad 2.2820-0.1150-0.2097 \quad 0.0130-0.0409 \quad 0.1530-0.0547-0.2150-0.0920-0.0050 \quad 0.0190 \quad 0.0410-0.1150 \quad 0.0510$

Fe5015 $4.9640-0.4440-0.1407-0.1400-0.0014-0.7460 \quad 0.1583-0.4480-0.1170-0.0780 \quad 0.0230 \quad 0.6160 \quad 0.8830-0.7110$

$\begin{array}{lllllll}\mathrm{Mg}_{1} & 0.0800 & 0.0150 & 0.0260-0.0050-0.0002-0.0320-0.0095 & 0.0330-0.0030-0.0020-0.0050-0.0060-0.0090-0.0180\end{array}$

$\begin{array}{lllllll}\mathrm{Mg}_{2} & 0.2050-0.0020 & 0.0167-0.0080 & 0.0008-0.0460-0.0019 & 0.0600-0.0050-0.0040-0.0040-0.0010 & 0.0040-0.0090\end{array}$

$\begin{array}{lllllllll}\mathrm{Mg} b & 3.2300-0.2680 & 0.0058-0.0890 & 0.0059-0.4470 & 0.1651 & 1.2620-0.0990-0.0480-0.0180 & -0.0110 & -0.0050 & 0.4790\end{array}$

$\begin{array}{lllllllll}\mathrm{Fe} 5270 & 2.7650-0.1710 & 0.0252-0.0700 & 0.0108-0.5900-0.0448-0.1810-0.0840-0.0440 & 0.1070 & 0.0090 & 0.7220-0.7290\end{array}$

Fe5335 $2.5330-0.2270-0.0200-0.1030-0.0178-0.5810-0.0030-0.1700-0.0880-0.0530-0.0290-0.0130 \quad 0.9090-0.8720$

Fe5406 $1.5470-0.1330-0.0004-0.0650-0.0039-0.3920-0.0078-0.0970-0.0610-0.0360-0.0310-0.0480 \quad 0.6130-0.6190$

Fe5709 $0.9590-0.1340-0.0566-0.0510-0.0244-0.2040 \quad 0.0108-0.0230-0.0300-0.0250-0.0080 \quad 0.0130 \quad 0.2280-0.2180$

Fe5782 $0.7840-0.0930-0.0307-0.0410-0.0125-0.1900-0.0106-0.0060-0.0170-0.0220-0.0100-0.0300 \quad 0.0540-0.2300$

$\begin{array}{lllllll}\mathrm{NaD} & 2.8340 & 0.0680 & 0.2474-0.0670 & 0.0521-0.8180-0.1204-0.0810-0.0410-0.0210-0.0580-0.0770 & 0.3990-1.1940\end{array}$

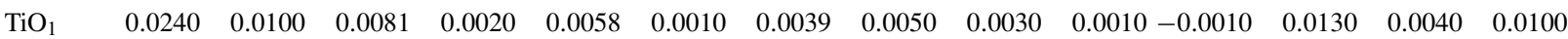

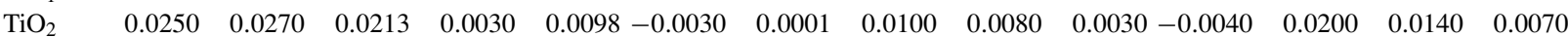

$\begin{array}{lllllllllllll}\mathrm{H} \delta_{A} & -0.3970 & 0.0210-0.6357 & 0.0430-0.2047 & 1.2520-0.2448 & 0.3900 & 0.2490 & 0.0890 & 0.2480 & -0.1100 & -2.4010 & 2.0720\end{array}$

$\begin{array}{llllllllll}\mathrm{H} \gamma_{A} & -3.6950-0.4320-1.2520 & 0.1800-0.1069 & 1.5670-0.1989 & 0.5510 & 0.2050 & 0.0970-0.0050-0.4030-1.6820 & 2.0990\end{array}$

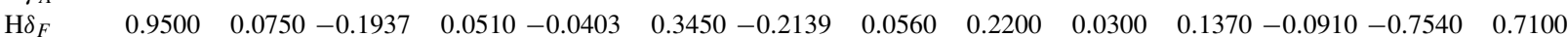

$\begin{array}{llllllllll}\mathrm{H} \gamma_{F} & -0.2360-0.2810-0.6841 & 0.0770-0.0494 & 0.6430-0.1163 & 0.1720 & 0.0500 & 0.0380-0.0150 & -0.0170 & -0.6380 & 0.9330\end{array}$

$-0.0009$

$-0.0021$

0.9642

$-0.0886$

$-0.7360$

0.1431

0.2122

$-0.0120$

$-0.3246$

0.6491

0.0248

0.0802

1.9536

$-0.0166$

$-0.1698$

$-0.1545$

0.0879

0.0204

$-0.3016$

0.0189

0.0175

0.5809

0.1432

0.1785

0.1314

Population age $12 \mathrm{Gyr}$, isochrone effects only

$\begin{array}{lllllllllll}\mathrm{CN}_{1} & 0.0230-0.0120-0.0055-0.0030 & 0.0007-0.0210 & 0.0055-0.0010-0.0020-0.0020 & 0.0000 & 0.0010 & 0.0340-0.0200\end{array}$

$\begin{array}{llllllllll}\mathrm{CN}_{2} & 0.0550-0.0140-0.0069-0.0030 & 0.0016-0.0210 & 0.0079 & 0.0010-0.0020-0.0020 & 0.0010 & 0.0020 & 0.0370-0.0210\end{array}$

$\begin{array}{llllllllllll}\mathrm{Ca} 4227 & 1.4710 & 0.0270 & 0.0843-0.0110 & 0.0059-0.0880 & 0.0026 & 0.0920 & 0.0620-0.0050-0.0050-0.0030 & 0.0960-0.0630\end{array}$

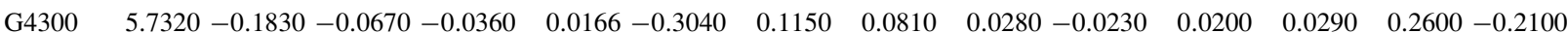

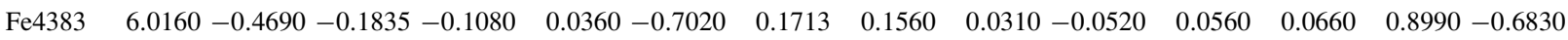

$\begin{array}{lllllllllll}\text { Ca4455 } & 1.7870-0.0250 & 0.0382-0.0190 & 0.0066-0.1700 & 0.0073 & 0.0480 & 0.0260-0.0100-0.0010 & 0.0020 & 0.2290-0.1720\end{array}$

$\begin{array}{llllllllllll}\mathrm{Fe} 4531 & 3.6960-0.0770 & 0.0233 & -0.0310 & 0.0125-0.2440 & 0.0177 & 0.0840 & 0.0390-0.0150 & 0.0070 & 0.0100 & 0.3030-0.2470\end{array}$

$\mathrm{C}_{2} 4668 \quad 4.9720-0.3210-0.0312-0.1210 \quad 0.0114-0.9250 \quad 0.1003-0.0170-0.0670-0.0750-0.0050 \quad 0.0200 \quad 1.6420-1.0790$

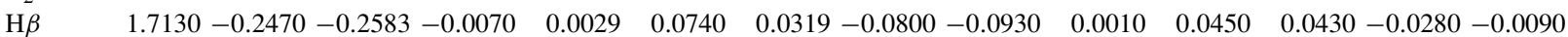

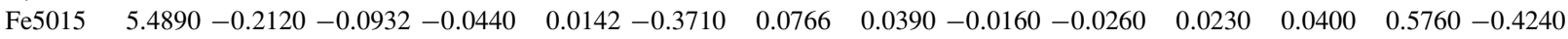

$\begin{array}{lllllllllllll}\mathrm{Mg}_{1} & 0.1160-0.0040 & 0.0027 & -0.0020 & 0.0012-0.0140 & 0.0023 & 0.0090 & 0.0040-0.0010 & 0.0000 & 0.0000 & 0.0160-0.0130\end{array}$

$\begin{array}{lllllllllll}\mathrm{Mg}_{2} & 0.2700-0.0090 & 0.0021 & -0.0030 & 0.0019-0.0230 & 0.0055 & 0.0120 & 0.0060-0.0010 & 0.0010 & 0.0020 & 0.0280-0.0210\end{array}$

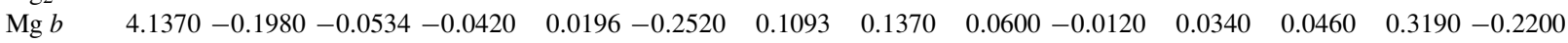

$\begin{array}{lllllllllll}\text { Fe5270 } & 3.2950-0.2250-0.1150-0.0400 & 0.0185-0.2420 & 0.0640 & 0.0720 & 0.0100-0.0150 & 0.0360 & 0.0400 & 0.3040-0.2530\end{array}$

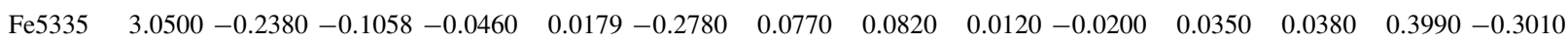

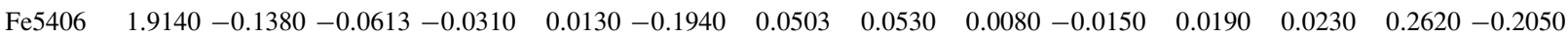

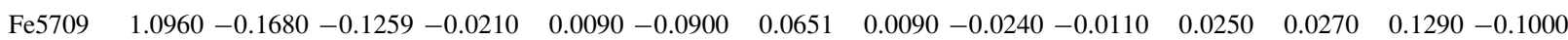

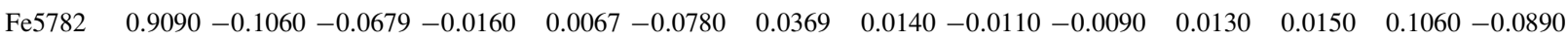

$\begin{array}{llllllllll}\mathrm{NaD} & 3.5260 & 0.1960 & 0.3170-0.0360 & 0.0104-0.4510-0.1153 & 0.1110 & 0.1090-0.0040-0.0380-0.0370 & 0.6460-0.5090\end{array}$

$\begin{array}{lllllllllll}\mathrm{TiO}_{1} & 0.0140 & 0.0290 & 0.0253 & 0.0020-0.0013-0.0010-0.0111-0.0020 & 0.0030 & 0.0000-0.0060-0.0060 & 0.0030-0.0020\end{array}$

$\begin{array}{lllllllllll}\mathrm{TiO}_{2} & 0.0020 & 0.0660 & 0.0572 & 0.0050-0.0018 & 9.4270-0.0238-0.0020 & 0.0090 & 0.0010-0.0110-0.0100 & 0.0090-0.0030\end{array}$

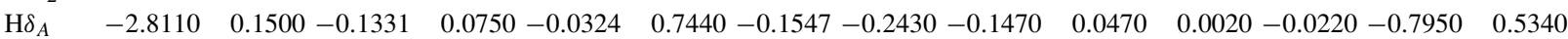

$\begin{array}{llllllllllll}\mathrm{H} \gamma_{A} & -6.5830 & 0.0410-0.2453 & 0.0640-0.0327 & 0.7250-0.1017 & -0.2980-0.1940 & 0.0430 & 0.0170-0.0040-0.6860 & 0.4890\end{array}$

$\begin{array}{llllllllll}\mathrm{H} \delta_{F} & -0.1300 & 0.0610-0.0451 & 0.0280-0.0136 & 0.2780-0.0637-0.0930-0.0590 & 0.0180-0.0030-0.0090 & -0.2650 & 0.1780\end{array}$

$\begin{array}{lllllllllll}\mathrm{H} \gamma_{F} & -1.8520 & 0.0330-0.1164 & 0.0310-0.0195 & 0.3440-0.0767-0.1770-0.1170 & 0.0180 & 0.0010-0.0100-0.2910 & 0.1910\end{array}$

0.0070

0.0080

0.1600

0.2130

0.3990

0.0980

0.1440

0.2410

$-0.1820$

0.1250

0.0150

0.0240

0.3210

0.1280

0.1490

0.1040

0.0350

0.0290

0.1800

$-0.0020$

$-0.0050$

$-0.6520$

$-0.6550$

$-0.2530$

$-0.3920$

Population age $12 \mathrm{Gyr}$, isochrone and spectral effects

$\begin{array}{llllllllllll}\mathrm{CN}_{1} & 0.0230 & 0.0100 & 0.0236 & 0.0310 & 0.0383-0.0540-0.0143-0.0110 & 0.0130-0.0040-0.0070 & 0.0030 & 0.0240-0.0540\end{array}$

$\begin{array}{llllllllllll}\mathrm{CN}_{2} & 0.0550 & 0.0080 & 0.0240 & 0.0330 & 0.0422 & -0.0620-0.0143-0.0140 & 0.0220-0.0030-0.0070 & 0.0050 & 0.0240 & -0.0590\end{array}$

$\begin{array}{lllllllllll}\mathrm{Ca} 4227 & 1.4710-0.2730-0.1853-0.2220-0.1875-0.1190 & 0.1724 & 0.0770 & 0.0110-0.0150 & 0.6420 & -0.0040 & 0.1600 & 0.4320\end{array}$

$\begin{array}{llllllll}\mathrm{G} 4300 & 5.7320 & 0.4910 & 0.6277-0.0310 & 0.0331-0.8630-0.3989-0.1610-0.1260-0.0290 & 0.1830 & 0.3650-0.2060-0.7810\end{array}$

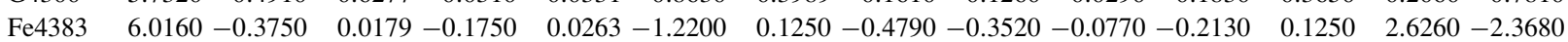

$\begin{array}{lllllllllll}\mathrm{C} 4455 & 1.7870-0.0740 & 0.0280-0.0430 & 0.0035-0.3180 & 0.0181-0.0160-0.0220-0.0190 & 0.0470 & 0.0530-0.0230-0.3530\end{array}$

$-0.0140$

$-0.0080$

1.3030

$-0.3130$

$-1.3250$

0.1470 
Table 5

Continued

\begin{tabular}{|c|c|c|c|c|c|c|c|c|c|c|c|c|c|c|c|}
\hline $\begin{array}{l}\text { Idex } \\
\text { ame }\end{array}$ & $\begin{array}{l}\text { Index } \\
\text { Value }\end{array}$ & $\begin{array}{l}\Delta I \\
(\mathrm{C})\end{array}$ & $\begin{array}{c}\Delta I \\
(\mathrm{C}+)\end{array}$ & $\begin{array}{l}\Delta I \\
(\mathrm{~N})\end{array}$ & $\begin{array}{c}\Delta I \\
(\mathrm{~N}+)\end{array}$ & $\begin{array}{l}\Delta I \\
(\mathrm{O})\end{array}$ & $\begin{array}{c}\Delta I \\
(\mathrm{O}+)\end{array}$ & $\begin{array}{c}\Delta I \\
(\mathrm{Mg})\end{array}$ & $\begin{array}{l}\Delta I \\
(\mathrm{Si})\end{array}$ & $\begin{array}{l}\Delta I \\
(\mathrm{~S})\end{array}$ & $\begin{array}{c}\Delta I \\
(\mathrm{Ca})\end{array}$ & $\begin{array}{c}\Delta I \\
(\mathrm{Ti})\end{array}$ & $\begin{array}{c}\Delta I \\
(\mathrm{Fe})\end{array}$ & $\begin{array}{l}\Delta I \\
(\alpha)\end{array}$ & $\begin{array}{c}\Delta I \\
(\alpha+)\end{array}$ \\
\hline & & & & & & & & & & & & & & & \\
\hline & & & & & & & & & & & & & & & \\
\hline$\beta$ & 30 & 0.2480 & 0.2812 & 0 & 8 & 0 & 12 & 90 & 50 & & & & 50 & & \\
\hline & & -0.4750 & 0.2428 & O & & & & -0.6200 & & & & & & & \\
\hline$g_{1}$ & 50 & 01 & .0307 & 00 & 9 & -0.0 & 3 & 0.0570 & 30 & 0 & 0 & 0 & 0 & -0 & \\
\hline $\mathrm{g}_{2}$ & 00 & -0.0010 & 3 & 0.0 & 0 & -0.0 & 1 & & -0.0 & 0.0 & 0 & & 0.0 & -0 . & \\
\hline $\mathrm{g} b$ & & 00 & 8 & -0.0 & 4 & -0.4 & 8 & 20 & 30 & 0 & & & 0 & & \\
\hline 5270 & 0 & -0.2320 & 8 & -0.0 & 9 & -0.6 & 7 & 0 & - & 0 & & & & 10 & 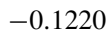 \\
\hline & & -0.3 & 1 & 0 & & & & & 0 & & & & & & \\
\hline & & & & & & & & & & & & & & & \\
\hline & & -0.1 & 0. & $-0 .($ & & - & & & 0 & & & & & -0 & \\
\hline 5782 & & -0.1 & -0. & -0 . & & -0 & & & -0 . & 0 & & & & -0 & \\
\hline $\mathrm{aD}$ & & & & -0.0 & & -0 & - & -0 & & - & - & & & -1 . & -0 \\
\hline $\mathrm{iO}_{1}$ & 140 & 0.0210 & 0.0 & 0.0020 & 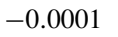 & 10 & -0. & -0 . & 20 & -0. & -0 & & 00 & & \\
\hline $\mathrm{TiO}_{2}$ & 0.0020 & 0.0540 & 0.0479 & 0.0 & 10 & -0.0 & -0.0 & -0.0 & & & -0 . & & & & \\
\hline$\delta_{A}$ & -2.8110 & -0.0710 & 0.5336 & -0.0450 & & & & & & & & & & & \\
\hline$\gamma_{A}$ & & -0.5090 & 0.9420 & & & & & & & & -0.0620 & & & & \\
\hline & & & & & & & -0.0 & & & & & & & & \\
\hline $\mathrm{H} \gamma_{F}$ & -1.8520 & -0.3450 & -0.5269 & 0.0450 & -0.0228 & 0.7050 & 0.1915 & 0.1800 & 0.0320 & 0.0250 & -0.0530 & -0.0540 & -0.6990 & 0.9790 & 0.516 \\
\hline
\end{tabular}

Notes. 1. The units of $\mathrm{CN}_{1}, \mathrm{CN}_{2}, \mathrm{Mg}_{1}, \mathrm{Mg}_{2}, \mathrm{TiO}_{1}, \mathrm{TiO}_{2}$ are mag. That of the rest are $\AA$. 2. Index value in the second column is the index value of the solar-scaled case. 3. $\Delta I=$ index value of each element-enhanced case - index value of solar-scaled. 4. $\mathrm{C}+, \mathrm{N}+, \mathrm{O}+$, and $\alpha+$ are those element-enhanced cases at fixed [Fe/H], whereas the rest are element-enhanced cases at fixed Z. 5. C and C+ are 0.2 dex enhanced cases and $\alpha+$ is 0.4 dex enhanced case, while the rest of the cases are 0.3 dex enhanced cases. $6 . \alpha$-elements are $\mathrm{O}, \mathrm{Ne}, \mathrm{Mg}, \mathrm{Si}, \mathrm{S}, \mathrm{Ca}$, and $\mathrm{Ti}$ in this study.

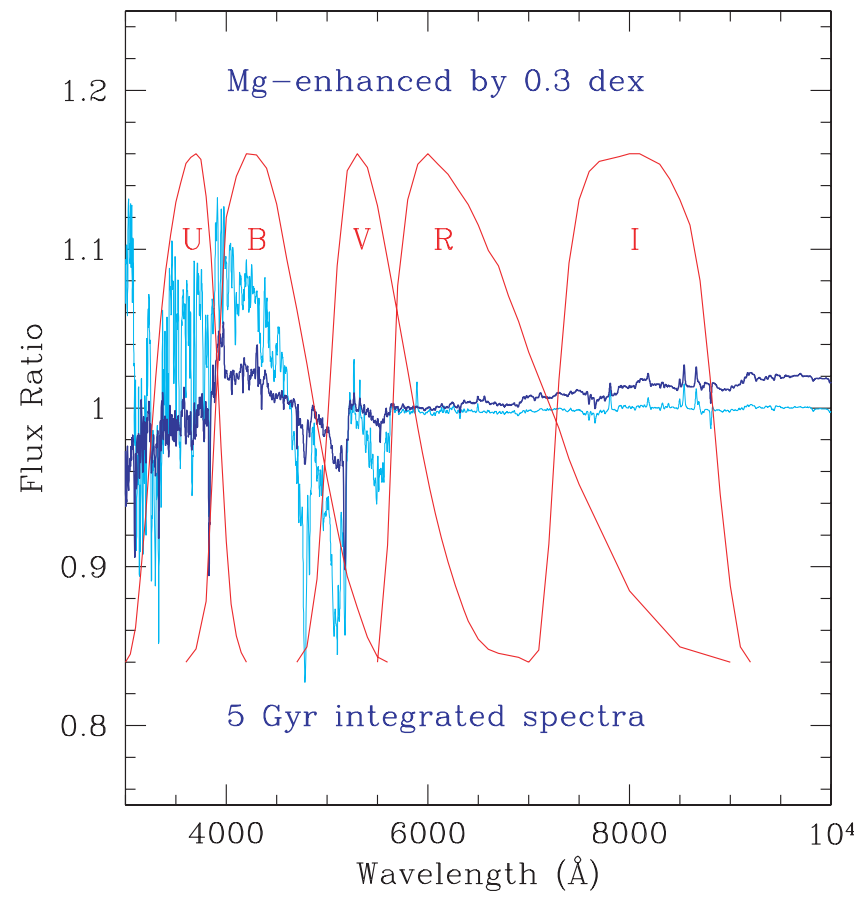

Figure 4. Same as Figure 3, but here the thicker line is the flux ratio between the solar-scaled and the Mg-enhanced spectra of the $5 \mathrm{Gyr}$ integrated spectra at solar metallicity. It is normalized at $6000 \AA$. The color changes because of the Mg-enhancement that we read from Table 4 can be vividly seen here (see the text).

(A color version of this figure is available in the online journal.)

near the regions of the $\mathrm{H} \beta$ and the $\mathrm{Mg} b$ lines, respectively. For the solar parameter we employ $T_{\text {eff }}=5777 \mathrm{~K}, \log g=4.44$, and $[\mathrm{Fe} / \mathrm{H}]=0$, and for Arcturus $T_{\text {eff }}=4290 \mathrm{~K}, \log g=1.9$, and $[\mathrm{Fe} / \mathrm{H}]=-0.7$ with $[\alpha / \mathrm{Fe}]=+0.4$ dex (e.g., Peterson et al.
1993; Griffin \& Lynas-Gray 1999; Carretta et al. 2004; Fulbright et al. 2007; Koch \& McWilliam 2008). The top panels are the solar spectra and the bottom panels are Arcturus. The thicker (black) lines are the observations and the thinner (red) ones are the synthetic. The degraded low-resolution spectra, Gaussiansmoothed to $8 \AA \mathrm{FWHM}$ or about $200 \mathrm{~km} \mathrm{~s}^{-1}$ — somewhat better than the best Lick-system resolution, are overlaid on top of the high-resolution spectra. We investigate the individual element effects over a rather broad wavelength region for the Lick indices and broadband colors in this paper. In this context, it is useful to find that the notable mismatches between the synthetic and the observed spectra in the high-resolution comparisons are smeared out and become subtle at low resolution. ${ }^{13}$

Table 1 presents some comparisons between synthetic colors and empirical color behavior for the stars in the temperature range $4000-6000 \mathrm{~K}$. Columns $2-5$ refer to synthetic spectra, and Columns 6-8 refer to the empirical calibration of Worthey \& Lee (2008). The synthetic spectra were converted to $U B V R_{C} I_{C}$ colors as in Worthey (1994) using Bessell's (1990) filter responses zeroed to Vega's colors. There is at least a 10 mmag uncertainty just from that procedure, and probably at least 20 mmag for $U-B$. The "dwarf" is $\log g=4.5$, and the "giant" is $\log g=2.5$. Columns 2 and 6 present the colors for the star listed, synthetic and empirical, respectively. The most serious mismatches are the $U-B$ color for the $6000 \mathrm{~K}$ dwarf and for the $4000 \mathrm{~K}$ and $5000 \mathrm{~K}$ giants. These facts suggest some room for improvement in model atmospheres and line lists (especially at shorter wavelengths).

The remaining columns present color changes induced by either an abundance increase of 0.3 dex or a temperature increase of $250 \mathrm{~K}$ (color changes in millimagnitudes). Column

\footnotetext{
${ }^{13}$ Due to assumptions about scattering vs. absorption in the SSG synthesis code, the bottoms of the saturated lines are artificially flattened. Some more comparisons between our synthetic spectra and Arcturus can be found at http://astro.wsu.edu/hclee/NSSPM_II_Arcturus.html
} 


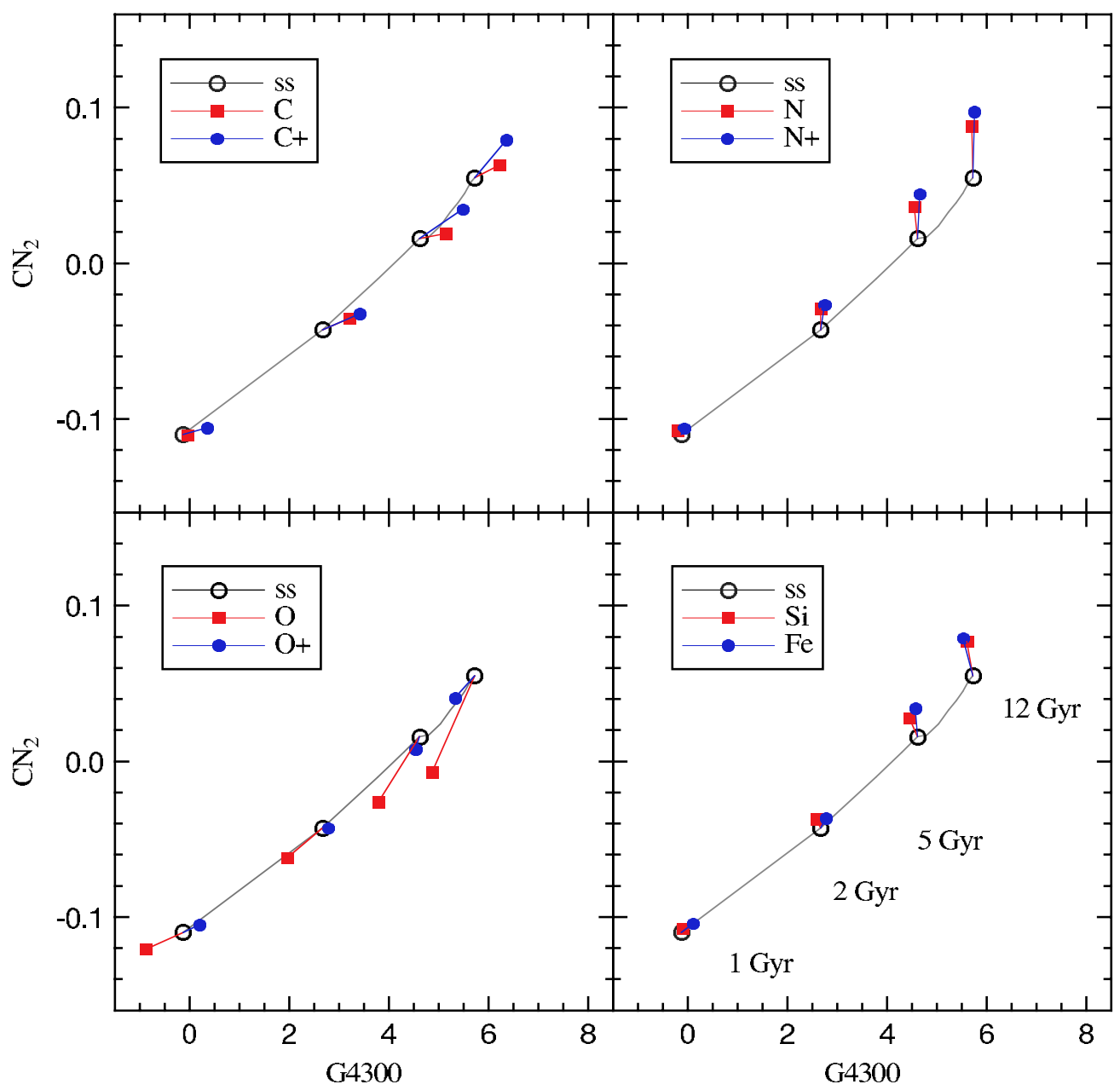

Figure 5. $\mathrm{CN}_{2}$ is plotted as a function of $\mathrm{G} 4300$ from $1 \mathrm{Gyr}$ to $12 \mathrm{Gyr}$ for simple stellar populations at solar metallicity. Carbon-enhanced (upper left), nitrogen-enhanced (upper right), oxygen-enhanced (lower left), and silicon-, iron-enhanced cases (lower right) at fixed $Z$ are depicted at 1, 2, 5, and 12 Gyr. C+, N+, and $\mathrm{O}+$ indicate the carbon-, nitrogen-, and oxygen-enhanced cases at fixed [Fe/H], respectively.

(A color version of this figure is available in the online journal.)

4 is a complete scaled-solar enhancement of 0.3 dex in every element and with new atmospheres calculated, while Column 3 is a 0.3 dex enhancement of only the 24 elements that are explicitly traced in the spectral library, a superset of those followed in the isochrone library. ${ }^{14}$ Comparison of Columns 3 and 4 indicates that, for the "heart" of the spectral library between 4000 and $6000 \mathrm{~K}$, the sum of the available one-by-one element tweaks approximately equals the scaled-solar analog operation. The list of 24 elements in linear combination thus appears to approximate the full-blown calculation of a spectrum in most cases. This allows the approximate calculation of a new spectrum at arbitrary composition in an eye blink rather than many minutes for a whole new synthetic spectrum calculation.

In general, the synthetic colors are within a few hundredths of a magnitude of the empirical colors and track the empirical color responses to abundance and temperature in an approximate way. The worst outlier is the $U-B$ color for the $6000 \mathrm{~K}$ giant, in which the color gets redder rather than bluer with increasing temperature (see also Martins \& Coelho 2007). Strong conclusions are not possible with this comparison, but the agreement is fairly encouraging.

14 They are C, N, O, Na, Mg, Al, Si, S, Cl, K, Ca, Sc, Ti, V, Cr, Mn, Fe, Co, $\mathrm{Ni}, \mathrm{Cu}, \mathrm{Zn}, \mathrm{Sr}, \mathrm{Ba}$, and $\mathrm{Eu}$.
Tables 2 and 3 show the spectral effects of element-byelement enhancements of 0.3 dex (except carbon which is increased by 0.15 dex, see footnote 12) on some selected color indices for the stars in the temperature range $4000-6000 \mathrm{~K}$. In Table 2, the elements were re-scaled to constant heavy-element fraction $Z$, while in Table $3, Z$ was allowed to increase. One technical subtlety is that, since neon is not tracked in the spectral library, the only effects that appear in Table 2 for $\mathrm{Ne}$ are due to the re-scaling, and there are no effects at all in Table 3. Table 3 can be visually explained by taking a look at the spectra. ${ }^{15}$ Figure 3 displays one example. Here, the flux ratio between 0.3 dex $\mathrm{Mg}$-enhanced spectra and that of the solar-scaled are shown for a $4000 \mathrm{~K}$ giant star. The $B-V$ becomes bluer (e.g., more $B$-band flux over $V$-band flux) and $V-R, V-I$ become redder (e.g., comparably more $R$ - and $I$-band fluxes over $V$-band flux). The $U-B$ also becomes similarly bluer just like $B-V$ because the $B$-band filter response curve goes all the way to $\sim 5400 \AA$ A. Now the effects of $\mathrm{Mg}$ that we read from Table 3 are clearly understood from this illustration. It is confirmed that $\mathrm{Mg}$ is the most important $\alpha$-element that influences the $U-B$ and $B-V$ colors as already described in Cassisi et al. (2004).

\footnotetext{
15 They are given at http://astro.wsu.edu/hclee/NSSPM_II_Color.html
} 


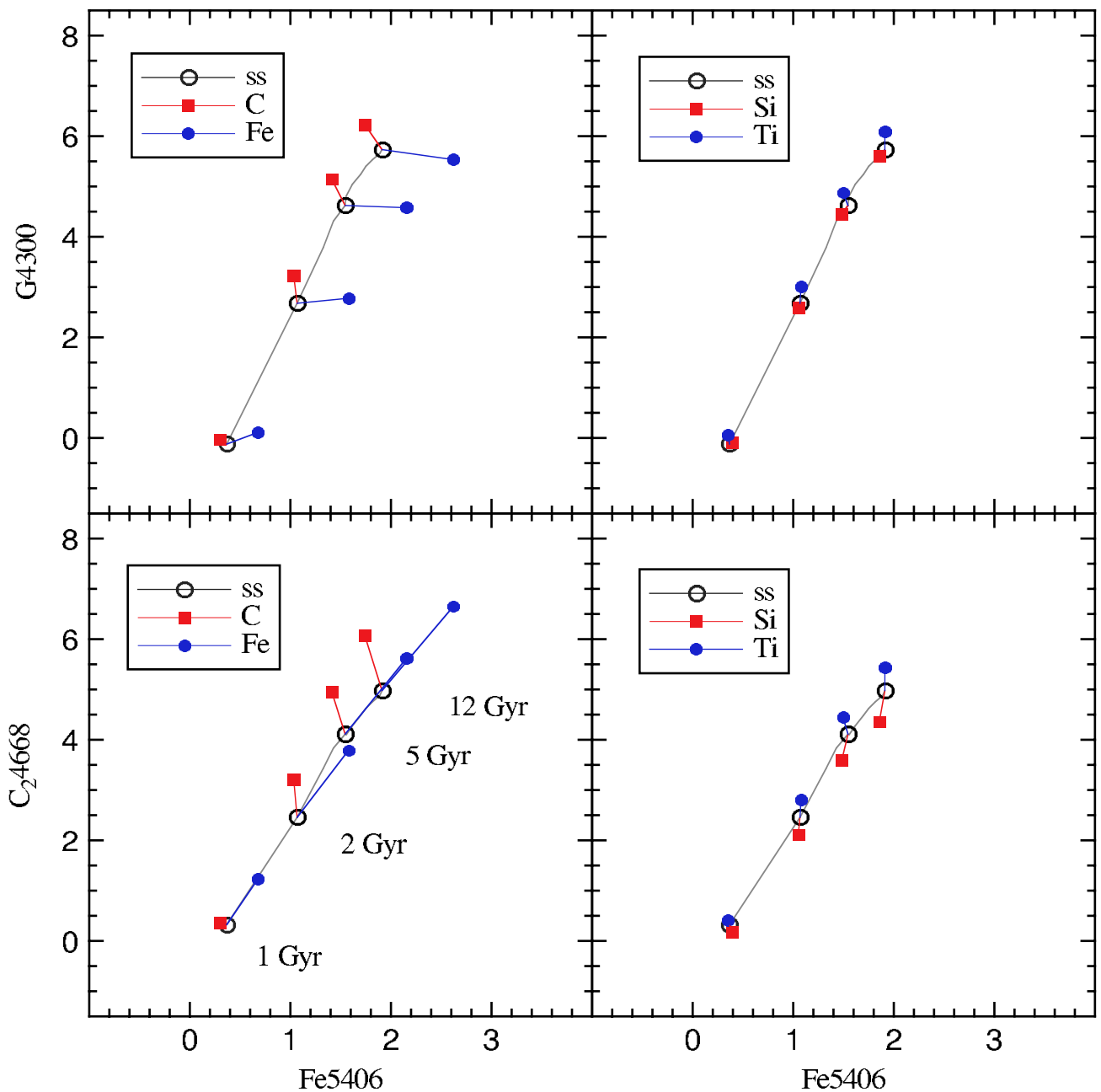

Figure 6. Fe5406 vs. G4300 (top) and $\mathrm{C}_{2} 4668$ (bottom). The left panels show carbon- and iron-enhanced cases, while the right panels display silicon- and titaniumenhanced cases. Note that $\mathrm{C}_{2} 4668$ is significantly affected both by carbon and iron. $\mathrm{C}_{2} 4668$ is further sensitive to silicon and titanium enhancement.

(A color version of this figure is available in the online journal.)

Comparing corresponding entries in the two tables, especially at the $6000 \mathrm{~K}$ giant, one sees that oxygen have little effect on the spectrum by themselves; it is the effect of the decrease of the rest of the elemental abundances that causes the bulk of the spectral change in the constant- $Z$ case. Unsurprisingly, it is also clear from the table that cooler stars are more susceptible to element-by-element effects than warmer stars, at least at optical wavelengths.

\subsection{Integrated-Light Color Results}

Table 4 presents color results for the isochrone-summed models assuming a Salpeter (1955) initial mass function and using the Worthey (1994) machinery for some disparate ages of populations. The bolometric correction (BC) row includes both isochrone effects and direct stellar spectral effects with the caveat that our synthetic spectra are limited in wavelength coverage, so the fluxes at given wavelengths were normalized to the older low-resolution Worthey (1994) flux library at the same $Z$. Thus, only effects that affect wavelengths between 3000 and $10000 \AA$ are considered, and there may be additional, small effects present that are not accounted for. However, in absolute value, all of the $\mathrm{BC}$ shifts are quite small, the largest being $6 \%$ for the case of all $\alpha$-elements enhanced. It is unlikely that element ratio effects will be of major concern for the mass estimation of clusters and galaxies from their luminosities (see also Cassisi et al. 2004).

The other immediate conclusion from Table 4 is that older populations show larger spectral effects due to element-byelement abundance changes. This is a straightforward consequence of the earlier conclusion that cooler stars are more sensitive; the light of older populations is dominated by stars that are cooler than those present in younger ones. ${ }^{16}$ Oxygen and neon tend to make the colors bluer, while species that contribute more to the lines in the spectrum generally make them redder. Exceptions can easily be explained if one knows where the lines contribute the most. For instance, magnesium has most of its absorption near $5100 \AA$, i.e., in the $V$ band, so adding $M g$ makes $B-V$ bluer while it makes $V-R$ and $V-I$ redder. The former, the bluer $B-V$, is also partly due to the increase in the flux in the region around $4000 \AA$ from the $\mathrm{Mg}$-enhanced spectra as shown in Figure 3 and described in Cassisi et al. (2004). The latter, the redder $V-R$ and $V-I$, also reflect the cooler red giant branch as illustrated in Figure 8 of Paper I. Figure 4 displays this Mg-enhanced integrated case at 5 Gyr (thick line) over the single-star case of a $4000 \mathrm{~K}$ giant (thin line). The populations

\footnotetext{
16 This last statement is valid for ages greater than $1 \mathrm{Gyr}$. For younger ages, IR fluxes from asymptotic giant branch (AGB) and TP-AGB stars complicate this status (see also Lee et al. 2007a).
} 


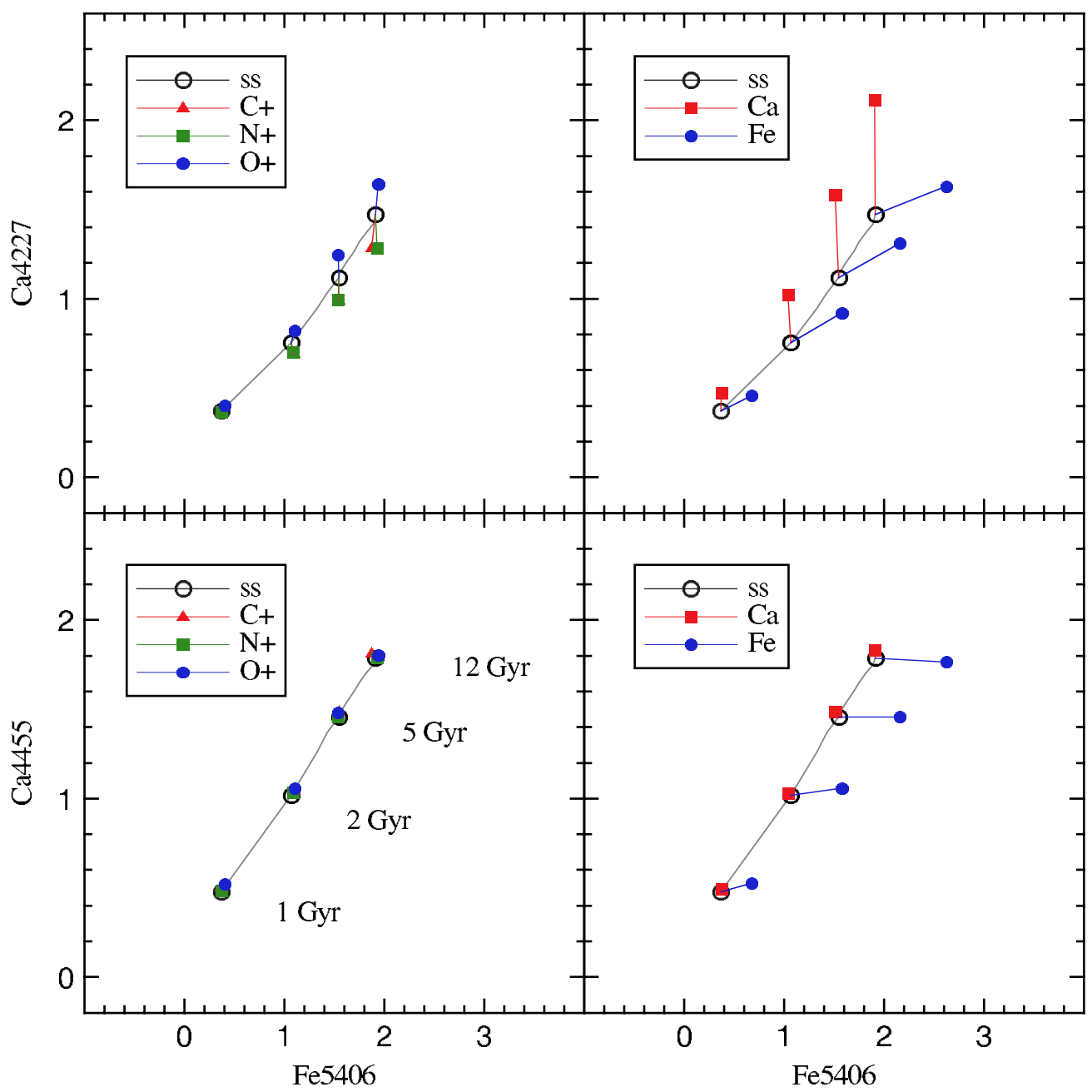

Figure 7. Fe5406 vs. Ca4227 (top) and Ca4455 (bottom). The left panels show carbon-, nitrogen-, and oxygen-enhanced cases at fixed [Fe/H], while the right panels depict calcium-, iron-enhanced cases at fixed Z. Ca4227 is predominantly calcium sensitive as well as a contribution from C, N, O, and Fe, while Ca4455 is hardly altered by those elements.

(A color version of this figure is available in the online journal.)

respond rather like the stars do, as can be seen from Figure 4. Based on this, one would suspect that isochrone-caused effects are relatively minor though non-negligible, and this conclusion will be confirmed and amplified in the following section.

\section{RESULTS IN LICK INDEX DIAGRAMS}

The effects of element-by-element enhancement on the Lick indices (Worthey et al. 1994) are described in this section. ${ }^{17}$ The synthetic indices are not very accurate in absolute predictions (see Korn et al. 2005; Serven et al. 2005), so we employ a differential approach in which the fitting functions of Worthey et al. (1994) and Worthey \& Ottaviani (1997) are used as the zero point, and delta-index information as a function of element ratio is incorporated via measuring the synthetic spectral library. This procedure is similar to that of previous investigations (e.g., Trager et al. 2000a, 2000b; Proctor \& Sansom 2002; Thomas et al. 2003; Lee \& Worthey 2005; Schiavon 2007) but more sophisticated since an entire grid of delta-index information was used. That is, 350 spectra at solar $Z$, plus similar grids for

\footnotetext{
17 In this study, we mostly describe the elemental effects on the Lick indices from the integrated spectra. Prompted by the referee's suggestion, however, we have looked into those elemental effects on the Lick indices at the stellar level. Some examples can be found at http://astro.wsu.edu/hclee/NSSPM_Lick.html
}

four other $Z$ values for this work, as opposed to two or three synthetic stars at solar abundance only for previous works.

To maintain an exact correspondence with Paper I, all the elements are 0.3 dex enhanced, except carbon, which is enhanced by 0.2 dex. ${ }^{18}$ In the cases of carbon, nitrogen, and oxygen enhancements, we investigate both at fixed total metallicity and at fixed $[\mathrm{Fe} / \mathrm{H}]$ (and $[\mathrm{R} / \mathrm{H}])$. The cases at fixed $[\mathrm{Fe} / \mathrm{H}]$ are denoted with plus sign in the figures (e.g., $\mathrm{C}+$, $\mathrm{N}+, \mathrm{O}+$ ). Also in the figures, solar-scaled solar metallicity predictions are connected by a solid line from $1 \mathrm{Gyr}$ to 12 Gyr and the element-enhanced cases are marked at 1,2,5, and 12 Gyr.

We have selected Fe5406 as a reference index in most plots. Among eight Lick iron indices (Fe4383, Fe4531, Fe5015, Fe5270, Fe5335, Fe5406, Fe5709, and Fe5782), we predict that Fe5406 is insensitive to every element except iron (see Figures

\footnotetext{
18 As footnote 12 says, the carbon-enhanced spectra are generated with 0.15 dex carbon enhancement. But the carbon-enhanced isochrones that we presented in Paper I are of 0.2 dex carbon enhancement. In order to be consistent, for the stellar population synthesis calculations, we extrapolated those 0.15 dex carbon-enhanced spectra to 0.2 dex enhancement in order to match the carbon-enhanced isochrones. Figure 1 of Paper I, in fact, needs to be corrected. The filled box for the case of carbon should be located near -0.04 instead of near 0 in terms of $[\mathrm{Fe} / \mathrm{H}]$.
} 


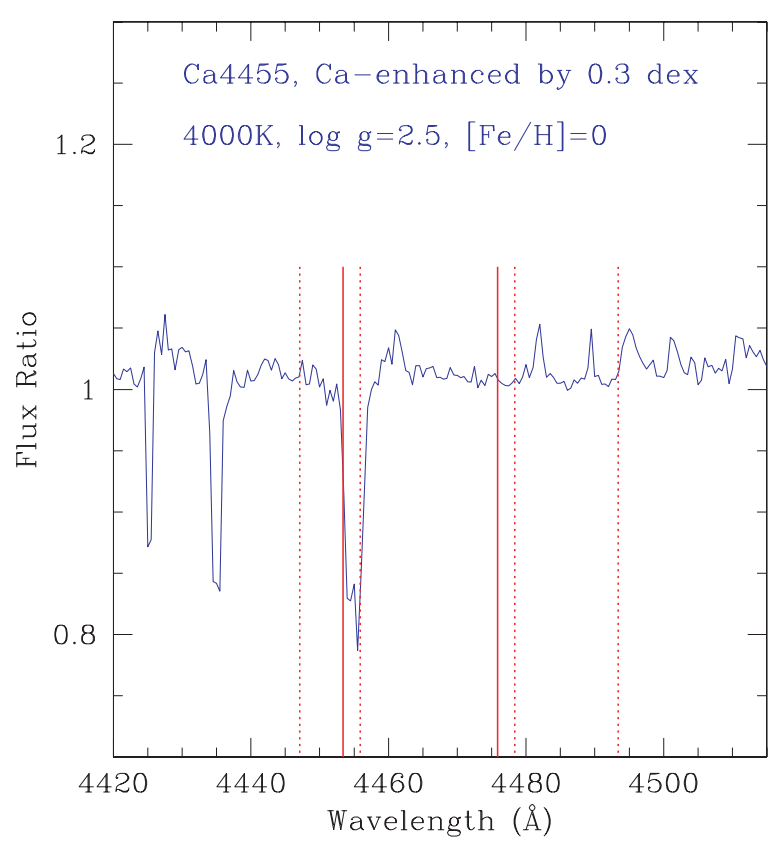

Figure 8. At $4000 \mathrm{~K}$ and $\log g=2.5$, solar-scaled and Ca-enhanced spectra are divided. The index definition of Worthey et al. (1994) for the Ca4455 index is depicted with straight lines (solid lines for the index bandpass and dotted lines for the pseudo-continua, respectively). Note that the strong $\mathrm{Ca}$ line feature is shared both by the index bandpass and the blue continuum that cancel out the calcium effect on the Ca4455 index.

(A color version of this figure is available in the online journal.)

10 and 11 and Table 5 and online spectra described in footnote 17), making it a convenient independent variable.

As has already been mentioned in the literature (Lee et al. 2007b), for most of the cases the isochrone effects are relatively minor compared to the stellar spectral effects (see also Schiavon 2007). For some cases, however, isochrone effects are nonnegligible and quite important (see $\mathrm{H} \beta$ in Figure 11; see also Figure 17 in Coelho et al. 2007).

It may be worthwhile to mention for clarity that "isochrone effects" indicate the temperature, luminosity, and stellar lifetime effects from element ratio changes, and, in the case of the ironenhanced mixture, the altered $[\mathrm{Fe} / \mathrm{H}]$ value that goes into the empirical fitting functions in our experiment. When calculating observables, we would still use a scaled-solar-ratio spectral library. These isochrones were presented in Paper I. In the present Paper II, we add the detailed spectral effects due to element-by-element enhancement at the stellar atmosphere/ stellar flux level. The "direct stellar spectral effects" are those that come purely from the emergent spectra with isochrones held fixed.

[Carbon and nitrogen: $C N_{2}$ ]: Lick indices $\mathrm{CN}_{1}$ and $\mathrm{CN}_{2}$ have identical central bandpasses, but $\mathrm{CN}_{2}$ has a narrower blue continuum which makes the $\mathrm{CN}_{2}$ somewhat less prone to abundance variations other than those due to elements $\mathrm{C}$ and $\mathrm{N}$. We compare $\mathrm{CN}_{2}$ with $\mathrm{G} 4300$ as carbon, nitrogen, oxygen, silicon, and iron are enhanced in Figure 5. The upper panels show that $\mathrm{CN}_{2}$ is both carbon and nitrogen sensitive. Nitrogen's effect is more prominent (partly because carbon is enhanced only by 0.2 dex compared to the 0.3 dex nitrogen enhancement). The bottom right panel also suggests that silicon and iron affect $\mathrm{CN}_{2}$ in a non-negligible way when stellar populations become older than 5 Gyr. We can tell from Table 5 that the silicon effect is mostly from the stellar spectra, while the iron effect is largely from the isochrones. The oxygen-enhanced case is displayed

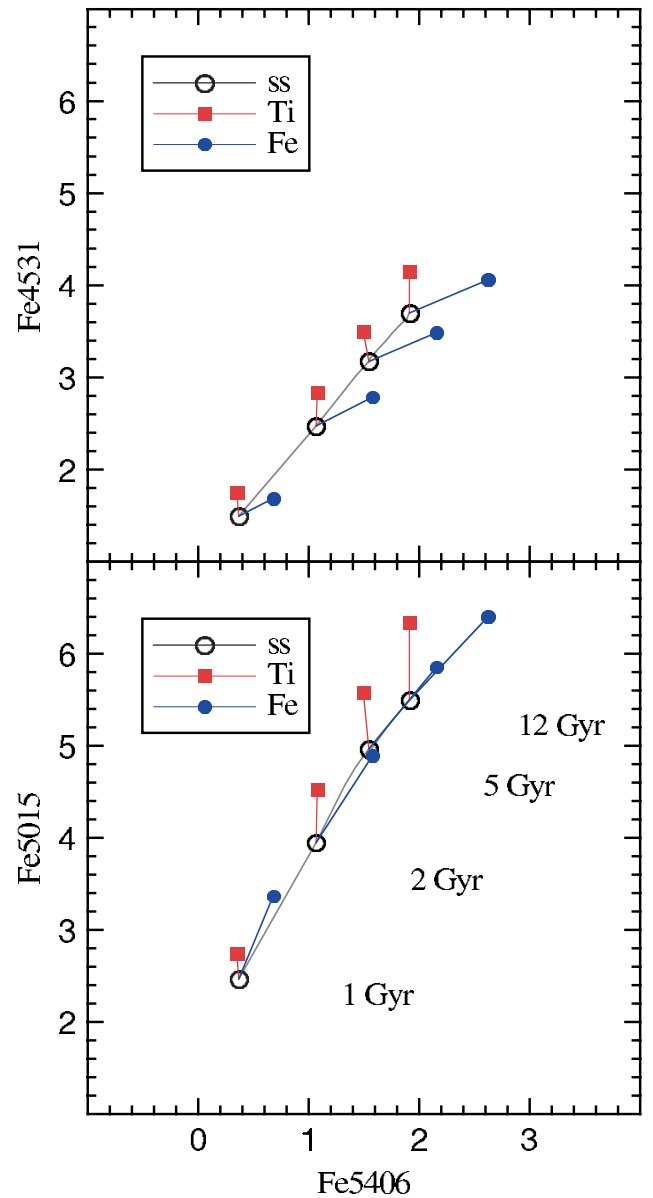

Figure 9. Fe5406 vs. Fe4531 (top) and Fe5015 (bottom). Titanium- and ironenhanced cases are depicted. Both of these Lick indices show a titanium sensitivity equal to that of iron.

(A color version of this figure is available in the online journal.)

in the bottom left panel. It shows that simply adding $\mathrm{O}$ alters $\mathrm{CN}_{2}$ and $\mathrm{G} 4300$ by a small amount (increases at young ages and decreases at old ages; the latter via consumption of more $\mathrm{C}$ into the $\mathrm{CO}$ molecule via molecular equilibrium balance ${ }^{19}$ ), but if $Z$ is held constant both indices decrease much more due to the displacement of $\mathrm{C}$ and $\mathrm{N}$ to lower abundance because of the O-enhancement and fixed sum. G4300 is N-insensitive. Also, we note that $\mathrm{CN}_{2}$ has little sensitivity to $\mathrm{Mg}-, \mathrm{S}-, \mathrm{Ca}-$, or Ti-enhancement.

[Carbon: $G 4300$ versus $C_{2} 4668$ ]: in Figure 6, G4300 and $\mathrm{C}_{2} 4668$ are plotted as a function of Fe5406. The left panels show $\mathrm{C}$ - and $\mathrm{Fe}$-enhanced models, while the right panels show the effects of Si- and Ti-enhancement. G4300 and $\mathrm{C}_{2} 4668$ are known to be good carbon indicators among Lick indices along with $\mathrm{CN}_{1}$ and $\mathrm{CN}_{2}$. The left panels show that this is indeed the case. In the bottom left panel, however, it can be seen that $\mathrm{C}_{2} 4668$ is also highly iron sensitive, as indicated by its former name, Fe4668 (Worthey 1994). ${ }^{20}$ Contrary to $\mathrm{C}_{2} 4668, \mathrm{G} 4300$

\footnotetext{
19 It is worth reiterating what Schiavon (2007) noted in his footnote 5. Carbon enhancement causes the opposite effect from oxygen enhancement and vice versa. This is because of the highest dissociation potential of $\mathrm{CO}$ molecule. Therefore, at cooler temperatures, more carbon translates to more $\mathrm{CO}$, resulting in less oxygen and vice versa.

20 We find from Table 5 that the Fe-sensitivity of $\mathrm{C}_{2} 4668$ (also $\mathrm{CN}_{2}$ and Fe5015) is mostly an isochrone effect. However, it is, in fact, not because of the temperature and/or luminosity changes, but because of the $[\mathrm{Fe} / \mathrm{H}]$ changes that go into the fitting function that we use for the index calculation. As one can
} 


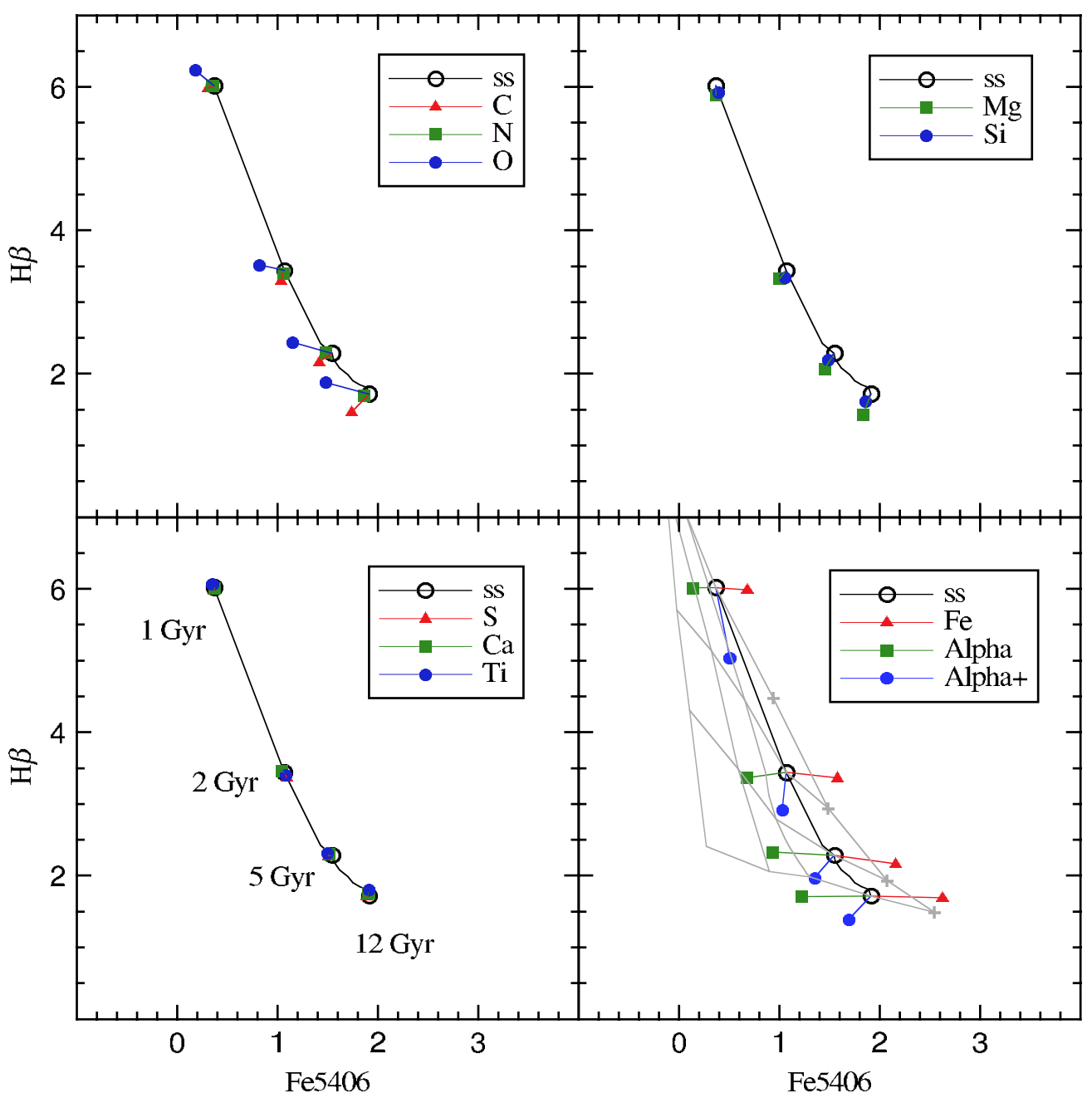

Figure 10. Displacements are shown in diagrams of $\mathrm{H} \beta$ as a function of Fe5406 as each chemical element is enhanced by 0.3 dex, except carbon, which is enhanced by $0.2 \mathrm{dex}$. In the lower-right panel, solar-scaled chemical mixtures of $[\mathrm{Fe} / \mathrm{H}]=-2.0,-1.0,-0.5,0.0$, and 0.5 are connected at $1,2,5$, and $12 \mathrm{Gyr}$. To guide the eye, the $[\mathrm{Fe} / \mathrm{H}]=0.5$ line is marked with plus signs at given ages. Also in the lower-right panel, the $\alpha$-element enhanced case at fixed $[\mathrm{Fe} / \mathrm{H}]$ is shown (see the text).

(A color version of this figure is available in the online journal.)

shows a negligible iron sensitivity. Furthermore, the right panels show that $\mathrm{C}_{2} 4668$ is influenced by $\mathrm{Si}$ and $\mathrm{Ti}$, whereas $\mathrm{G} 4300$ shows only slight Ti sensitivity. Both G4300 and $\mathrm{C}_{2} 4668$ show little sensitivity by $\mathrm{N}-, \mathrm{Mg}-$, $\mathrm{S}-$, or $\mathrm{Ca}$-enhancement.

The prospects for disentangling $\mathrm{C}, \mathrm{N}$, and $\mathrm{O}$ are now fairly good, because it appears that there is a lot of sensitivity among various indices. However, at the moment it appears that, with the indices available, there will be considerable degeneracy among the three quantities. In the future, adding $\mathrm{NH}, \mathrm{CH}$, and $\mathrm{CO}$ features may help considerably (e.g., Yong et al. 2008; Martell et al. 2008; Marmol-Queralto et al. 2008).

[Calcium: Ca4227 versus Ca4455]: Figure 7 compares Ca4227 and Ca4455 with Fe5406. Carbon-, nitrogen-, and oxygen-enhanced cases at fixed $[\mathrm{Fe} / \mathrm{H}]$ are displayed at the left panels, while the right panels depict calcium- and ironenhanced cases at fixed $Z$. It is clear from the upper-right panel of Figure 7 that Ca4227 is significantly boosted with calcium enhancement, the effect increasing with age. To a lesser degree, Ca4227 is also affected by C, N, O, and Fe. Carbon and nitrogen

find from Figure 1 of Paper $\mathrm{I},[\mathrm{Fe} / \mathrm{H}]=0.268$ for the Fe-enhanced isochrones compared to -0.225 for the $\alpha$-enhanced ones at constant solar metallicity, $Z$.

Clearly, future fitting-function work will need to be more meticulously defined in terms of abundance parameters, and not locked to $[\mathrm{Fe} / \mathrm{H}]$ necessarily. enhancements make Ca4227 weaker by $0.3 \AA$, whereas the oxygen enhancement does the opposite mostly due to their effects at the blue continuum (see also Prochaska et al. 2005).

Contrary to Ca4227, Ca4455 is hardly influenced by any of those elements. According to this study, Ca4455 is found to be the most element-enhancement-free Lick index. This is consistent with the previous findings by Tripicco \& Bell (1995) and Korn et al. (2005) although our presentation is based on a large spectral grid weighted by isochrones rather than three stars. One concern, however, is that two recent data sets of Milky Way globular clusters, by Cohen et al. (1998) and Puzia et al. (2002), both significantly disagree with theoretical model predictions and also each other (Figure 3 of Lee \& Worthey 2005). According to Tables 1-3 of Tripicco \& Bell (1995), Ca4455 has the strongest dependence on the bandpass placement (wavelength shift error) among Lick indices. This is because that the blue continuum and the index bandpass of Ca4455 share the strong $\mathrm{Ca}$ absorption line feature near $4455 \AA$ and consequently cancel out its effect, but only if the wavelength match is perfect. This phenomenon at the stellar level with $10 \mathrm{~km} \mathrm{~s}^{-1}$ velocity dispersion is displayed in Figure 8. Both $\mathrm{Ca} 4227$ and $\mathrm{Ca} 4455$ are rather insensitive to $\mathrm{Mg}-$, $\mathrm{Si}-$, $\mathrm{S}$-, or Ti-enhancement. 


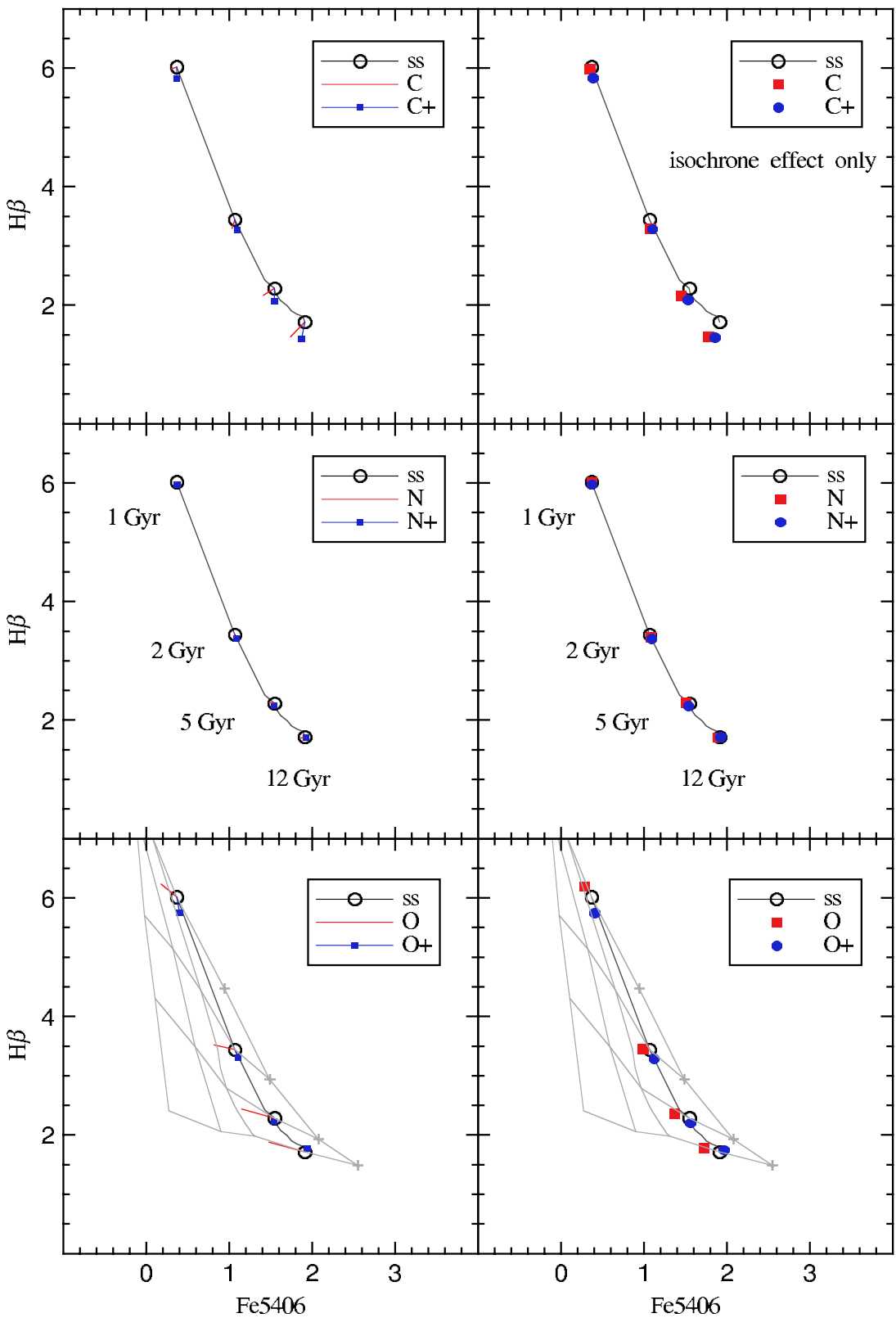

Figure 11. C-, N-, O-enhanced cases are shown from top to bottom in diagrams of $\mathrm{H} \beta$ as a function of Fe5406. The same solar-scaled chemical mixture grids with a range of metallicity and age are displayed here in the bottom panels as in the bottom panels of Figure 10. The left panels show the combination of isochrone and stellar spectral effects, while the right panels display the isochrone effects alone.

(A color version of this figure is available in the online journal.)

[Titanium: Fe4531 and Fe5015]: Fe4531 and Fe5015 are displayed in Figure 9 with Fe5406. TB95, Trager (1997), KMT05, LW05, and Serven et al. (2005) indicate that these two indices are titanium sensitive, and we confirm that their titanium sensitivity is indeed strong and almost comparable to the iron sensitivity. Fe5406, on the other hand, demonstrates little titanium sensitivity.

[Balmer lines: $H \beta, H \gamma_{A}, H \gamma_{F}, H \delta_{A}, H \delta_{F}$ ]: Balmer lines are widely used as an age indicator because of their nonlinear temperature sensitivity in stars, tracing better than many indices the temperature of the main-sequence turnoff. However, Lee \& Worthey (2005) and earlier work (Worthey et al. 1994; Thomas et al. 2004; Coelho et al. 2007) found that they are also abundance sensitive to some degree. In Figures 10-18, we look into their element-by-element sensitivity in detail.
Effects of the individual 10 chemical elements' enhancement on the $\mathrm{H} \beta$ and Fe5406 are shown in Figure 10. The effects of carbon, nitrogen, and oxygen enhancements are of great importance but they are relatively difficult to understand here because our experimental setup preserves the total metallicity. In other words, it is not straightforward to determine whether we are seeing the effects of $\mathrm{C}-, \mathrm{N}-$, and $\mathrm{O}$-enhancement or whether we are seeing other elements (such as $\mathrm{Mg}$ and $\mathrm{Fe}$ ) countereffects due to their depression. Hence, in Figure 11, we display $\mathrm{C}$-, $\mathrm{N}$-, and $\mathrm{O}$-enhancement cases both at the fixed total metallicity and at the fixed $[\mathrm{Fe} / \mathrm{H}]$ (the plus signs). It is seen from the left panels of Figure 11 that unlike nitrogen, oxygen at young ages and carbon at old ages influence $\mathrm{H} \beta$. The top and bottom right panels of Figure 11 illustrate that it is mostly the isochrone-level effects of carbon and oxygen enhancements that 


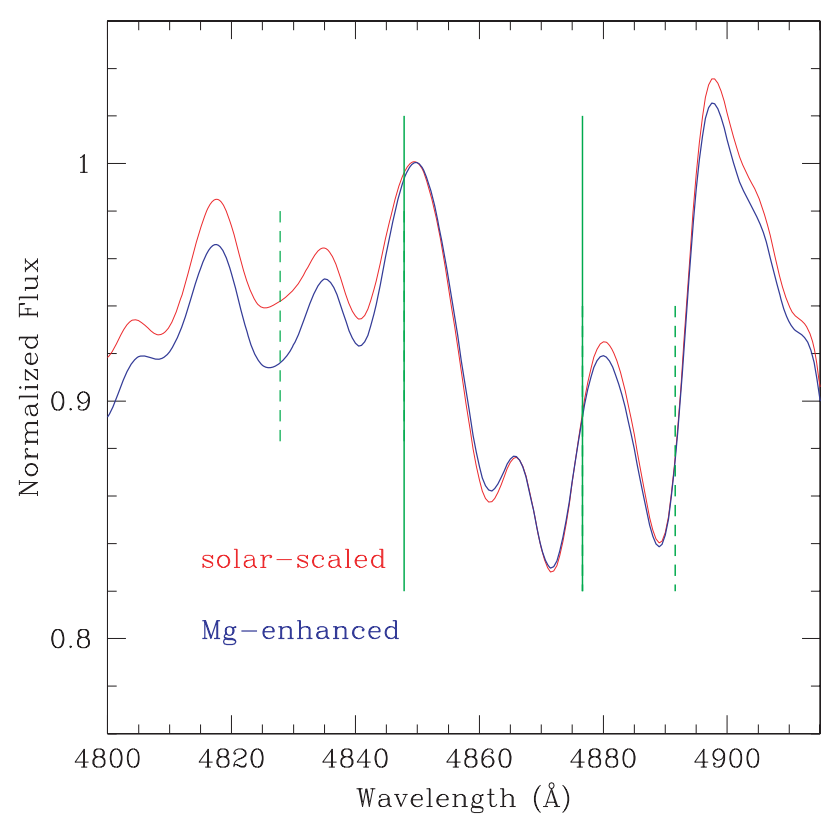

Figure 12. Solar-scaled and Mg-enhanced 12 Gyr integrated spectra near $\mathrm{H} \beta$ (4861 $\AA$ ). Note that the $\mathrm{Mg}$ lines make the blue-continuum levels significantly lower, which consequently make the $\mathrm{H} \beta$ index strengths weaker as we see in the upper-right panel of Figure 10.

(A color version of this figure is available in the online journal.)

affect $\mathrm{H} \beta$. Figure 10 further shows that $\mathrm{H} \beta$ is similarly altered by the Mg-enhancement and in this case it is mostly due to the synthetic spectra (see also Table 5 and Figure 12).

In the lower-right panel of Figure 10 (also in the bottom panels of Figure 11), a grid with a range of metallicity $([\mathrm{Fe} / \mathrm{H}]=-2.0$, $-1.0,-0.5,0.0$, and 0.5$)$ is displayed at $1,2,5$, and 12 Gyr. These additional calculations are of solar-scaled chemical mixtures (Dotter et al. 2007a). We have also depicted $\alpha$-element enhanced cases both at the fixed $Z$ and at the fixed $[\mathrm{Fe} / \mathrm{H}]$ (Alpha+) ${ }^{21}$ It is intriguing to note that the effect due to the Fe-enhancement by 0.3 dex is seen over the $[\mathrm{Fe} / \mathrm{H}]=0.5$ grid line. This is because these grids only reflect the mere isochrone effects of iron variation with solar-scaled chemical mixtures, while the $0.3 \mathrm{dex} \mathrm{Fe}$-enhancement case shows both isochrone and spectral effects combined.

We find that the $\mathrm{H} \beta$ becomes weaker in the $\alpha$-element enhanced case at fixed $[\mathrm{Fe} / \mathrm{H}]$ mostly because of the effects of $\mathrm{Mg}$ (both isochrone and spectral as one could see from Table 5), while it stays nearly unchanged in the $\alpha$-element enhanced case at the fixed $Z$ mostly because of the reflection of depression of Fe. At $12 \mathrm{Gyr}$, a comparison of integrated spectra uncovers that adding $\mathrm{Mg}$ brings down the blue continuum, making $\mathrm{H} \beta$ weaker, ${ }^{22}$ but adding Fe brings down the central bandpass as well as the red continuum, the net effects of which tend to cancel out effects from Fe. Alpha-enhancement at fixed $Z$ mostly reflects the decrease of $\mathrm{Fe}$ due to dilution rather than overt, direct spectral effects from $\alpha$-elements. They are displayed in Figures 12-14. The solid lines are the index bandpass and the dashed lines are the blue- and the redcontinuum edges.

\footnotetext{
21 The $\alpha$-element enhanced case at the fixed [Fe/H] (Alpha+) is $[\alpha / \mathrm{Fe}]=+0.4$ dex at $[\mathrm{Fe} / \mathrm{H}]=0$ using the Dotter et al. (2007a). The $[\mathrm{Fe} / \mathrm{H}]$ of the $\alpha$-element enhanced case at the fixed $Z$ (Alpha) is -0.225 using Paper I. 22 This was hinted in Tripicco \& Bell (1995).
}

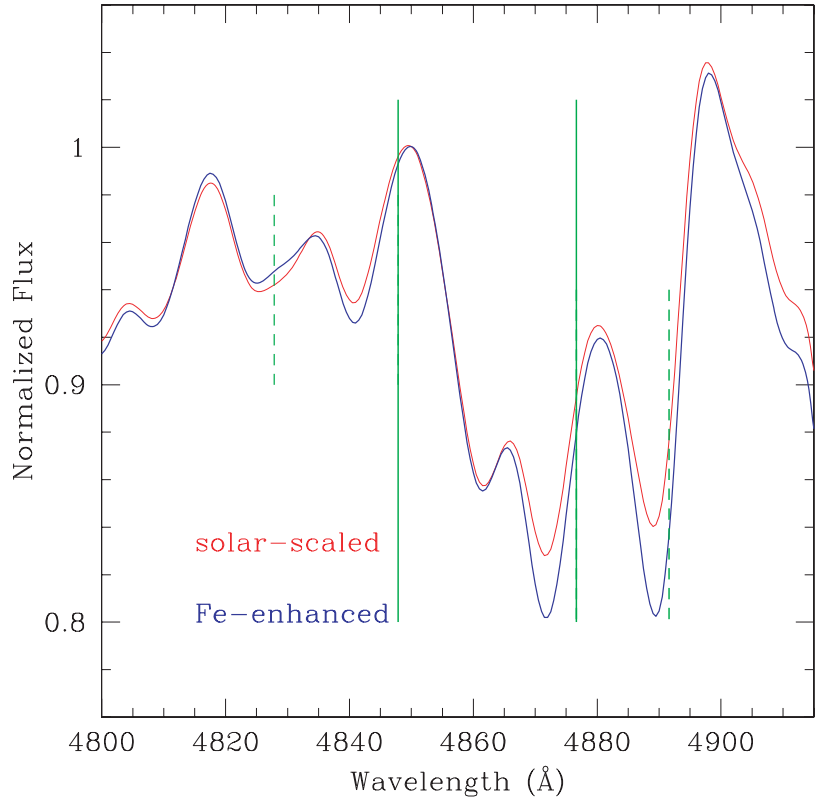

Figure 13. Solar-scaled and Fe-enhanced $12 \mathrm{Gyr}$ integrated spectra near $\mathrm{H} \beta$ (4861 $\AA$ ). Note that Fe lines make the index bandpass and the red-continuum levels significantly lower, which consequently cancel out their effects on the $\mathrm{H} \beta$ index strengths as we see in the lower-right panel of Figure 10.

(A color version of this figure is available in the online journal.)

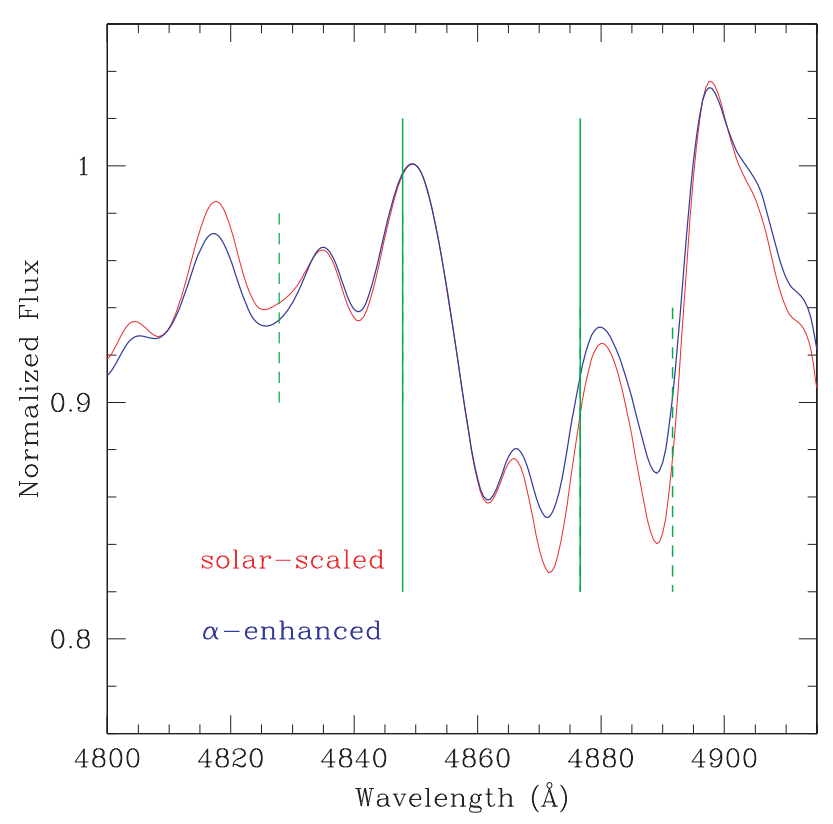

Figure 14. Solar-scaled and the $\alpha$-enhanced $12 \mathrm{Gyr}$ integrated spectra near $\mathrm{H} \beta$ (4861 $\AA$ ). Note that the $\alpha$-enhanced spectra at constant $Z$ mostly reflect the Fe depression effect which is the opposite of what is seen in Figure 13 and they affect the $\mathrm{H} \beta$ index strengths little as we see in the lower-right panel of Figure 10.

(A color version of this figure is available in the online journal.)

Figures 15 and 16, in contrast, display that both $\mathrm{H} \gamma$ and $\mathrm{H} \delta$ become mildly $(\sim 0.7 \AA)$ stronger with $\mathrm{Mg}$-enhancement. Furthermore, the top panels of Figures 15 and 16 illustrate that the broader $A$ indices $\left(\mathrm{H} \gamma_{A}, \mathrm{H} \delta_{A}\right)$ show sensitivity to Feenhancement and become significantly weaker with increasing age, by up to $3 \AA$ at $12 \mathrm{Gyr}$ for the case of the $\mathrm{H} \delta_{A}$. This is mostly due to spectral effects, as can be seen from Table 5 . 


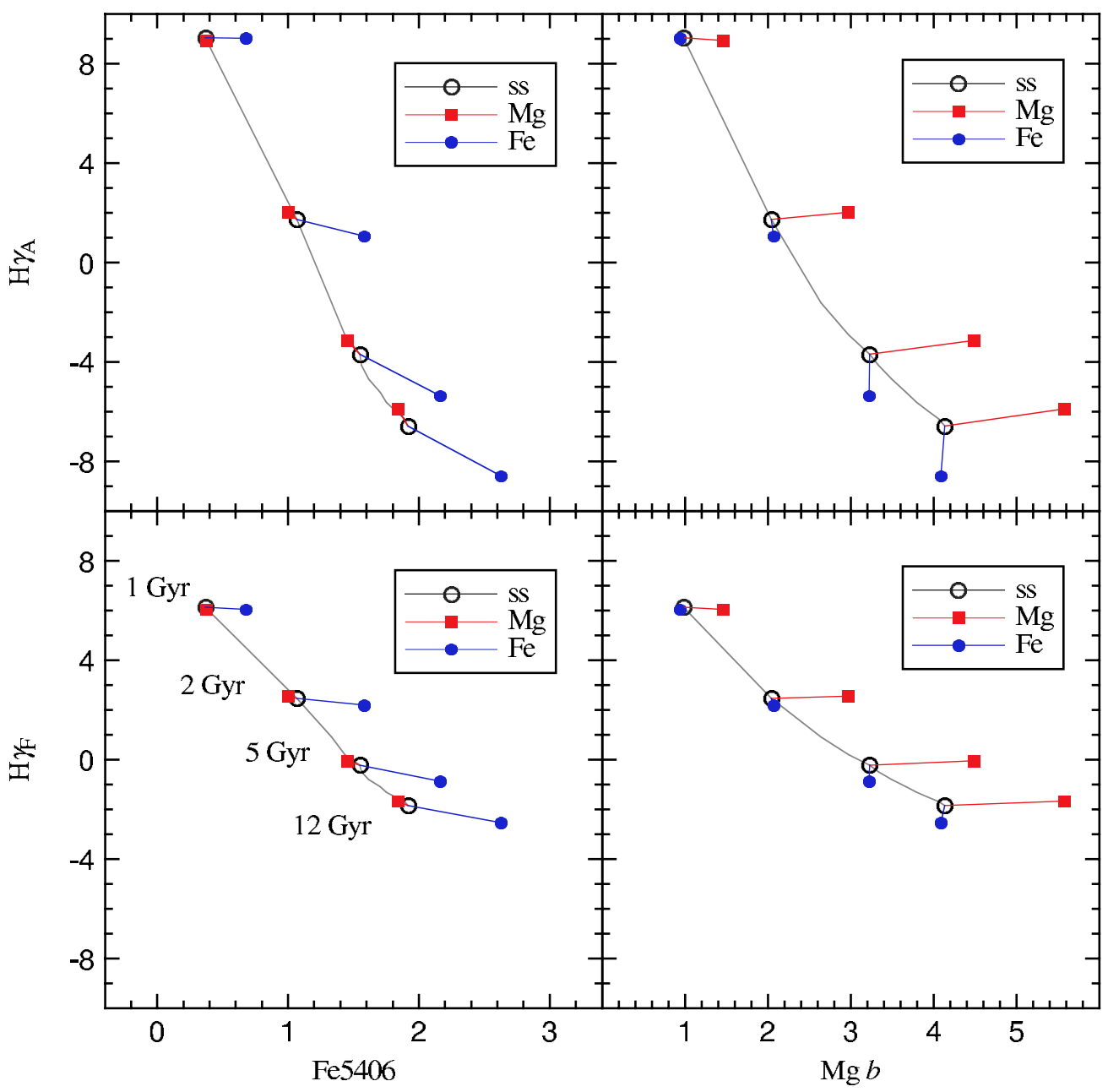

Figure 15. $\mathrm{H} \gamma_{A}$ (top) and $\mathrm{H} \gamma_{F}$ (bottom) are compared with Fe5406 (left) and $\mathrm{Mg} b$ (right). Clearly, $\mathrm{H} \gamma_{A}$ is significantly affected by iron enhancement.

(A color version of this figure is available in the online journal.)

They are illustrated in Figures $17(\mathrm{H} \gamma)$ and $18(\mathrm{H} \delta)$. From this experiment, it seems that for the high-order Balmer lines, the $F$ indices $\left(\mathrm{H} \gamma_{F}, \mathrm{H} \delta_{F}\right)$ are less prone to abundance changes because of their narrower index definition and therefore may possibly serve as more robust age indicators. Furthermore, it is found that $\mathrm{H} \delta_{F}$ among Balmer lines has the least sensitivity to $\mathrm{C}, \mathrm{N}$, and O (see also Schiavon et al. 2002; Prochaska et al. 2007).

In Figure 19, we show some selected diagrams for illustrating element enhancement. In the upper-left panel, $\mathrm{CN}_{2}$ and $\mathrm{G} 4300$ are compared and the effects of carbon and nitrogen enhancements are depicted. The plot shows that $\mathrm{CN}_{2}$ is sensitive to both carbon and nitrogen. $\mathrm{CN}_{2}$ index values go up by $0.04 \mathrm{mag}$ at 12 Gyr with 0.3 dex nitrogen enhancement here. G4300, in contrast, is primarily a carbon-sensitive index. Ca4227 and G4300 are contrasted in the lower-left panel of Figure 19 and the effects of sulfur, calcium, and titanium are illustrated. It is seen that Ca4227 is significantly affected by calcium enhancement whereas G4300 is not much altered by anything but carbon. In the upper-right panel, $\mathrm{Mg} b$ and $\mathrm{Fe} 5406$ are compared and the effects of magnesium and silicon are shown. It is clear that $\mathrm{Mg}$ $b$ is notably affected by the magnesium enhancement. $\mathrm{H} \beta$ and $\mathrm{Fe} 5406$ are contrasted in the lower-right panel and, as we have seen in Figures 10 and 13, $\mathrm{H} \beta$ is not Fe-enhancement sensitive, while Fe5406 is significantly affected by Fe.
Figure 20 is basically same as Figure 19, but here the isochrone effects are decoupled from those of synthetic spectra. The lines show the combination of isochrones and stellar spectral effects, while the points depict the isochrone effects alone at 1, 2, 5, and 12 Gyr. It is seen again from Figure 20 that the synthetic spectra make the spectral lines stronger and/ or weaker, while isochrones play a comparatively minor role. The displacement of points in the bottom right panel is mostly due to the different $[\mathrm{Fe} / \mathrm{H}]$ values that go into the index value calculations rather than the isochrone changes (see Figure 1 of Paper I).

\section{SUMMARY AND DISCUSSION}

We have commenced this project to make stellar population models which incorporate flexible chemistry, so that almost any interesting chemical mixture can be interpolated. Paper I dealt with the effects on the stellar evolution models, examining the temperature and luminosity changes due to the altered opacities when 10 chemical elements are individually tweaked to the end of the red giant branch. In this paper, we combine those isochrone effects with the stellar spectral effects in order to investigate their mixed effects on the integrated spectrophotometric indices as well as in their integrated spectra themselves. 


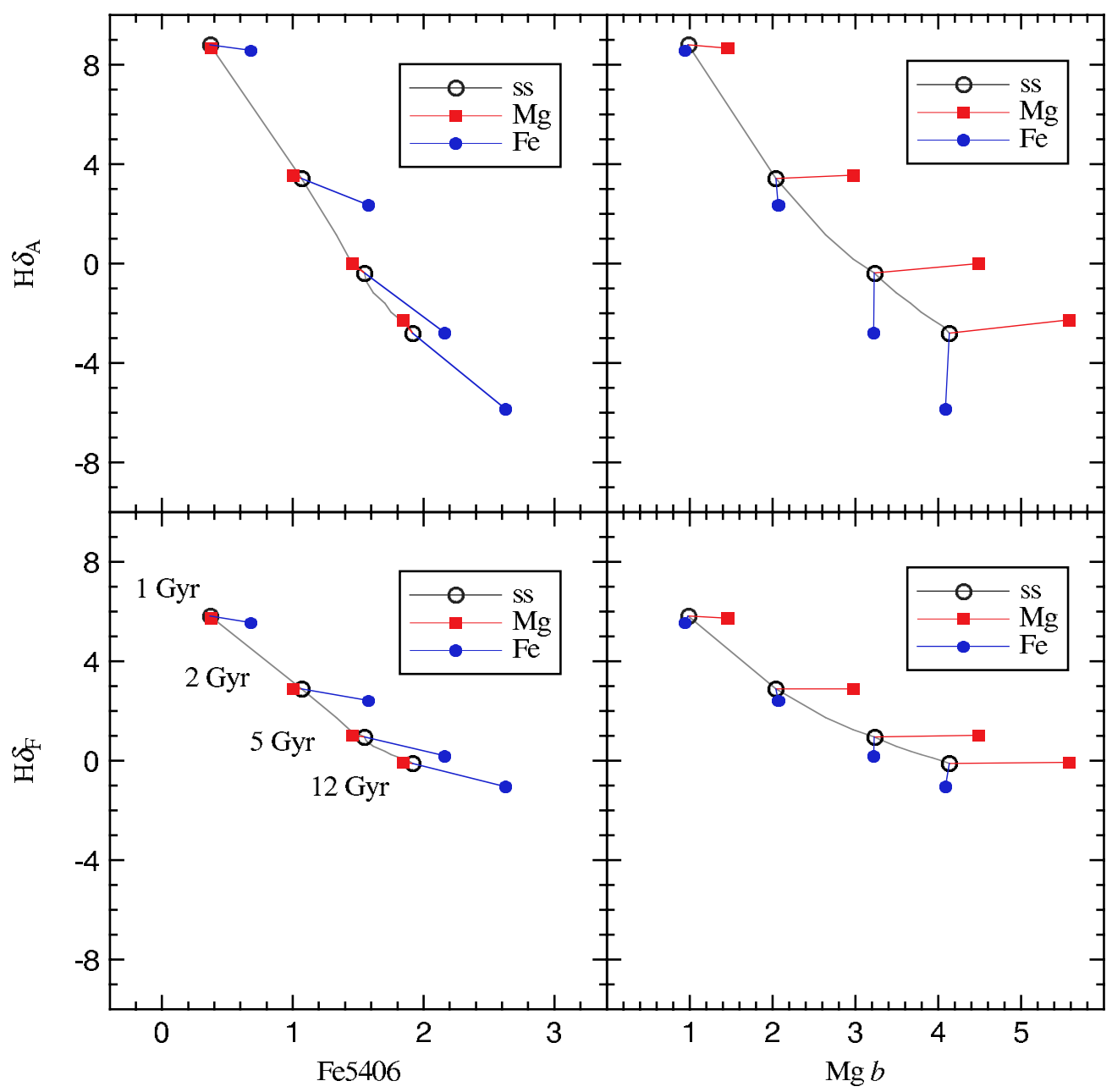

Figure 16. Same as Figure 15, but $\mathrm{H} \delta_{A}$ (top) and $\mathrm{H} \delta_{F}$ (bottom) are compared with Fe 5406 (left) and $\mathrm{Mg} b$ (right). Again, clearly $\mathrm{H} \delta_{A}$ is significantly affected by iron enhancement.

(A color version of this figure is available in the online journal.)

We again emphasize here that the models in this study are incomplete in terms of inclusion of all stellar evolutionary phases and should not be used blindly when comparing to real stellar populations until the helium-burning phases are properly incorporated. A version with a full range of metallicity and with horizontal branch and AGB stars included is planned. Comparison of our models with observations of Virgo cluster galaxies will be presented as well (J. L. Serven et al. 2009, in preparation).

Within our spectral coverage (3000-10000 $\AA$ ), we have investigated the broadband color behaviors in the $U B V R_{C} I_{C}$ filter set. As one would expect, older populations show larger spectral effects due to element-by-element abundance changes. This mostly reflects temperature effects. We have also confirmed that $\mathrm{Mg}$ is the most important $\alpha$-element that shapes the $U-B$ and $B-V$ colors as already depicted in Cassisi et al. (2004).

From our investigation of Lick indices using the integrated spectra, we find that (1) $\mathrm{CN}_{2}$ is a useful nitrogen indicator once we have good carbon abundances from $\mathrm{G} 4300$ and $\mathrm{C}_{2} 4668$, but good silicon (and also titanium for the $\mathrm{C}_{2} 4668$ ) abundances are also needed, (2) $\mathrm{Ca} 4227$ is a robust calcium indicator with some good constraints of C, N, and O, (3) Fe4531 and Fe5015 are very useful titanium indicators where an independent iron abundance is provided, (4) $\mathrm{Mg} b$ and Fe5406 are good magnesium and iron indicator, respectively, and (5) the variation of individual elements affects the Balmer lines. We defer the investigation of $\mathrm{NaD}$ and $\mathrm{TiO}_{1}$ and $\mathrm{TiO}_{2}$ indices until we have the Naenhanced isochrones and can model $\mathrm{TiO}$ molecular effects with confidence. Below, we illuminate some points that we have described here with the help of full spectra.

Fe4531 and Fe5015: from Figure 9 we see that both Fe4531 and Fe5015 prove to be good titanium indicators. They are almost equally sensitive to titanium and iron. Figures 21-23 show the SAURON (Bacon et al. 2001) spectral range, 4810$5350 \AA$, that includes $\mathrm{H} \beta, \mathrm{Fe} 5015, \mathrm{Mg}_{1}, \mathrm{Mg} b, \mathrm{Fe} 5270$, and a part of Fe5335. Here the integrated spectra of solar-scaled, $\mathrm{Mg}-, \mathrm{Fe}-$, and Ti-enhanced cases are shown at $2 \mathrm{Gyr}$ with $300 \mathrm{~km} \mathrm{~s}^{-1}$ velocity dispersion normalized at $4750 \AA$. $\mathrm{H} \beta$ is not influenced much by the enhancement of these elements at this age, as Figure 10 also shows. $\mathrm{Mg} b$ is mostly sensitive to $\mathrm{Mg}$, as Figure 21 shows, while Fe5015, Fe5270, and Fe5335 are notably sensitive to Fe-enhancement, although Fe5015 is equally sensitive to titanium, as Figures 22 and 23 display.

Moreover, we have found that the Fe-enhanced and Tienhanced spectra show their centroids at different wavelengths in the case of Fe4531, opposite sides from the scaled-solar spectra, because of different locations of iron and titanium 


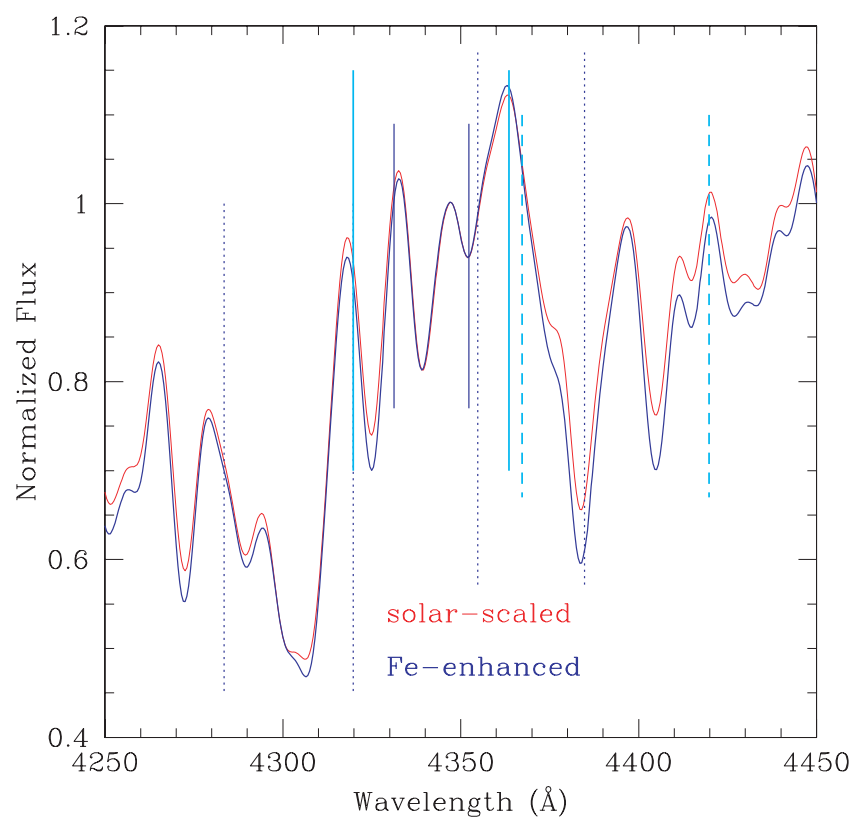

Figure 17. Solar-scaled and Fe-enhanced $12 \mathrm{Gyr}$ integrated spectra near $\mathrm{H} \gamma$ (4341 $\AA$ ). The solid lines and the dashed lines are the index bandpasses (the broader ones are the $A$ indices, while the narrower ones are the $F$ indices) and the continua regions for the $A$ indices, respectively, and the dotted lines are the continua regions for the $F$ indices. $\mathrm{H} \gamma_{A}$ and $\mathrm{H} \gamma_{F}$ have the same blue continuum. Note that the iron lines make the red-continuum levels significantly lower, which consequently make the $\mathrm{H} \gamma$ index strengths weaker, especially for the broader $\mathrm{H} \gamma_{A}$ index as we see in the upper panels of Figure 15.

(A color version of this figure is available in the online journal.)

lines in their index bandpass. Figure 24 displays this case. ${ }^{23} \mathrm{We}$ have preliminary hints from high-S/N, high-resolution spectra of Virgo cluster galaxies taken at the Kitt Peak 4 m telescope (J. L. Serven et al. 2009, in preparation) that galaxies with the higher velocity dispersion tend to have their centroids at the longer wavelengths, indicating higher Ti-enhancements. If confirmed, this would indicate a Ti- $\sigma$ relation, similar to the well-known $\mathrm{Mg}-\sigma$ relation (see also Milone et al. 2000).

$\mathrm{H} \beta$ : the bottom right panel of Figure 10 shows that, in an $\alpha$-enhanced mixture at the fixed $[\mathrm{Fe} / \mathrm{H}]$ (Alpha+), $\mathrm{H} \beta$ becomes weaker. This sounds a bit different from the conclusion of LW05 where $\mathrm{H} \beta$ is $\alpha$-element insensitive. As we can see from Table 5, the effects that we see from the bottom right panel of Figure 10 are mostly isochrone effects, especially at young ages. The $\mathrm{H} \beta$ index becomes weaker because of the decrease of temperature of stellar isochrones from the $\alpha$-enhanced case at fixed $[\mathrm{Fe} / \mathrm{H}]$ (see also Coelho et al. 2007; Schiavon 2007). LW05 used only solarscaled isochrones at solar and super-solar metallicities, although these authors employed modified stellar spectra because of the $\alpha$-enhancement.

Moreover, it is worth emphasizing that $\alpha$-enhanced isochrones at fixed $Z$ are now no longer significantly hotter than the solar-scaled ones. This has been revealed by Paper I, in its Figure 11, after incorporating the latest development of lowtemperature opacities by Ferguson et al. (2005). If this behavior is indeed true, it will certainly affect the conclusions of earlier works (e.g., Thomas \& Maraston 2003) that are based upon the

\footnotetext{
23 This phenomenon at the stellar level with higher resolution can be seen at http://astro.wsu.edu/hclee/Fe45_4000mp00gp25_ti.pdf and http://astro.wsu.edu/hclee/Fe45_4000mp00gp25_fe.pdf
}

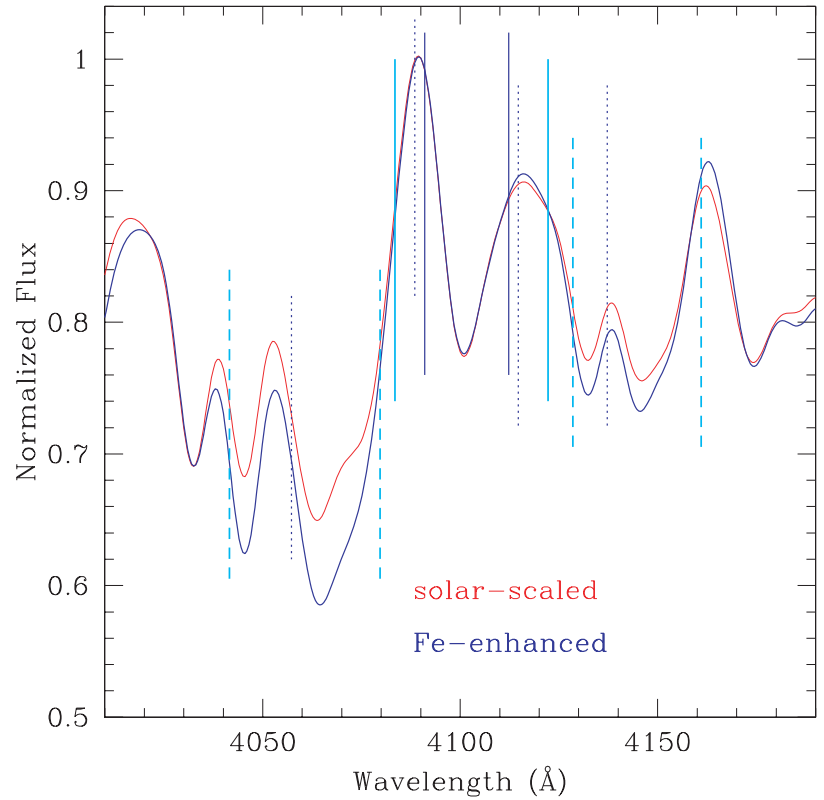

Figure 18. Solar-scaled and Fe-enhanced 12 Gyr integrated spectra near $\mathrm{H} \delta$ (4102 A). Symbols are the same as in Figure 17. Note that the iron lines make the continua levels significantly lower, which consequently make the $\mathrm{H} \delta$ index strengths weaker, especially for the broader $\mathrm{H} \delta_{A}$ index as we see in the upper panels of Figure 16.

(A color version of this figure is available in the online journal.)

Salasnich et al. (2000) isochrones, which were later shown by Weiss et al. (2006) to present problems.

$\mathrm{H} \gamma$ and $\mathrm{H} \delta$ : Fe-enhancement makes these indices significantly weaker, especially for the broader indices, $\mathrm{H} \gamma_{A}$ and $\mathrm{H} \delta_{A}$ (see also Graves \& Schiavon 2008). Figures 17 and 18 show that this is because of $\mathrm{Fe}$ lines that inhabit the continuum region. It is suggested therefore that the narrower indices $\left(\mathrm{H} \gamma_{F}\right.$ and $\left.\mathrm{H} \delta_{F}\right)$ may serve as better age indicators in case the stellar systems are of wildly different chemical mixtures.

Our goals are to narrow down the uncertainties of mean age estimations to $10 \%$ when derived from a single integrated light spectrum. We believe that we can reach these goals as we are able to take into account the effects from individual chemical elements. The ultimate goal then obviously would be the understanding of the chemical enrichment and star formation histories of galaxies, stepping away from the study of mean ages and mean metallicities to begin to tackle the more realistic problem of multi-age, multi-metallicity stellar populations as they exist in galaxies. We believe that several efforts such as STARLIGHT (Cid Fernandes et al. 2005), SPECKMAP (Ocvirk et al. 2006), and MOPED (Panter et al. 2007), will be even more useful once they use sophisticated stellar population models with flexible chemistry as inputs.

We are grateful to the referee, Ricardo Schiavon, for a thorough reading which greatly helped us to improve the paper. We also thank Jim Rose, Jon Fulbright, and Andreas Koch for useful discussions on the Mg-enhancement of the metal-rich cool giants. Support for this work was provided by the NSF through grant AST-0307487, the New Standard Stellar Population Models (NSSPM) project. H.-c.L. also acknowledges J P Blakeslee's support via NASA grant. P.C. acknowledges the support by the European Community under a Marie Curie International Incoming Fellowship. 

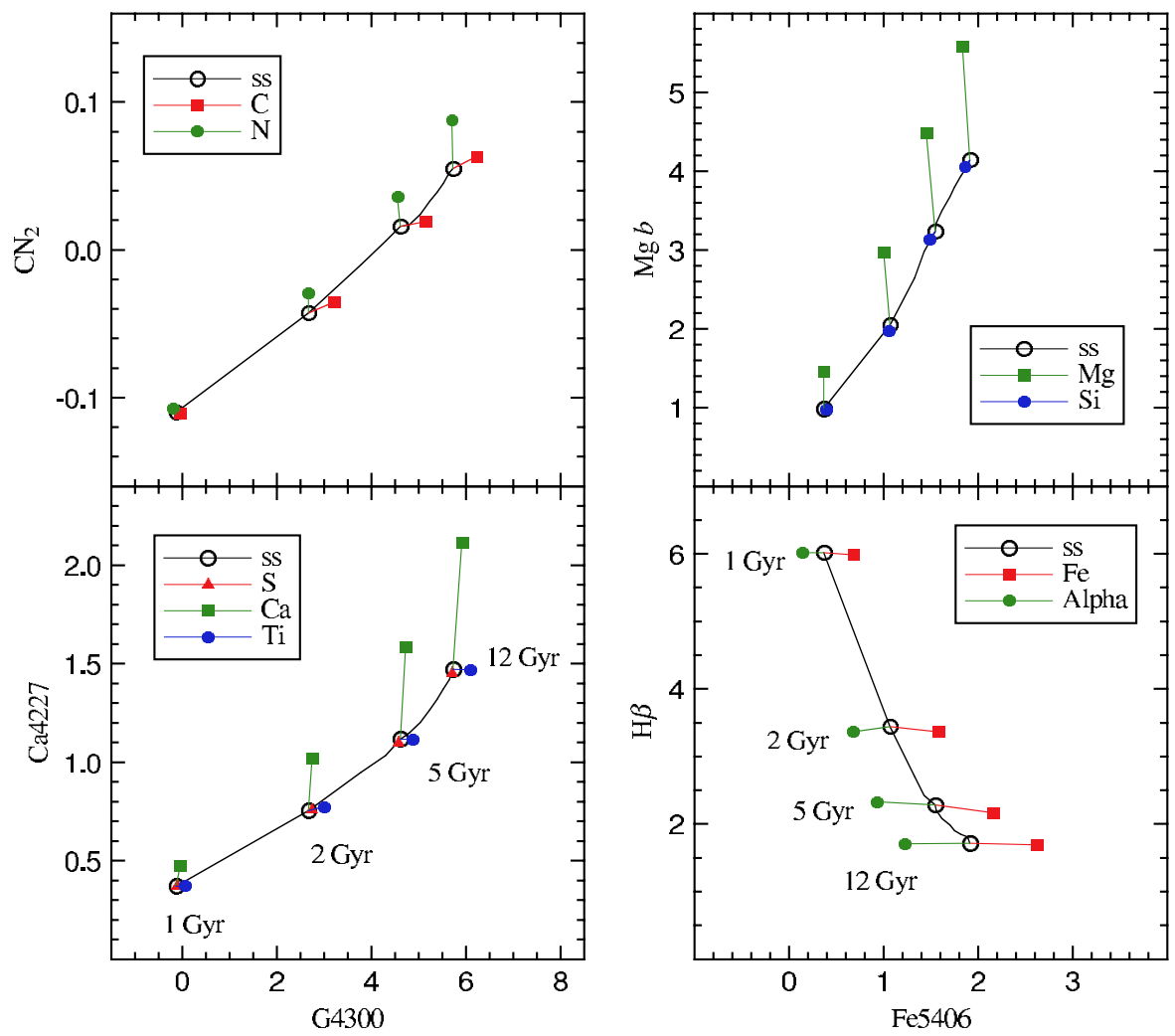

Figure 19. Most conspicuously element-sensitive cases are collectively displayed here. Left: $\mathrm{G} 4300$ vs. $\mathrm{CN}_{2}$ (top) and Ca4227 (bottom). Right: Fe5406 vs. Mg $b$ (top) and $\mathrm{H} \beta$ (bottom).

(A color version of this figure is available in the online journal.)
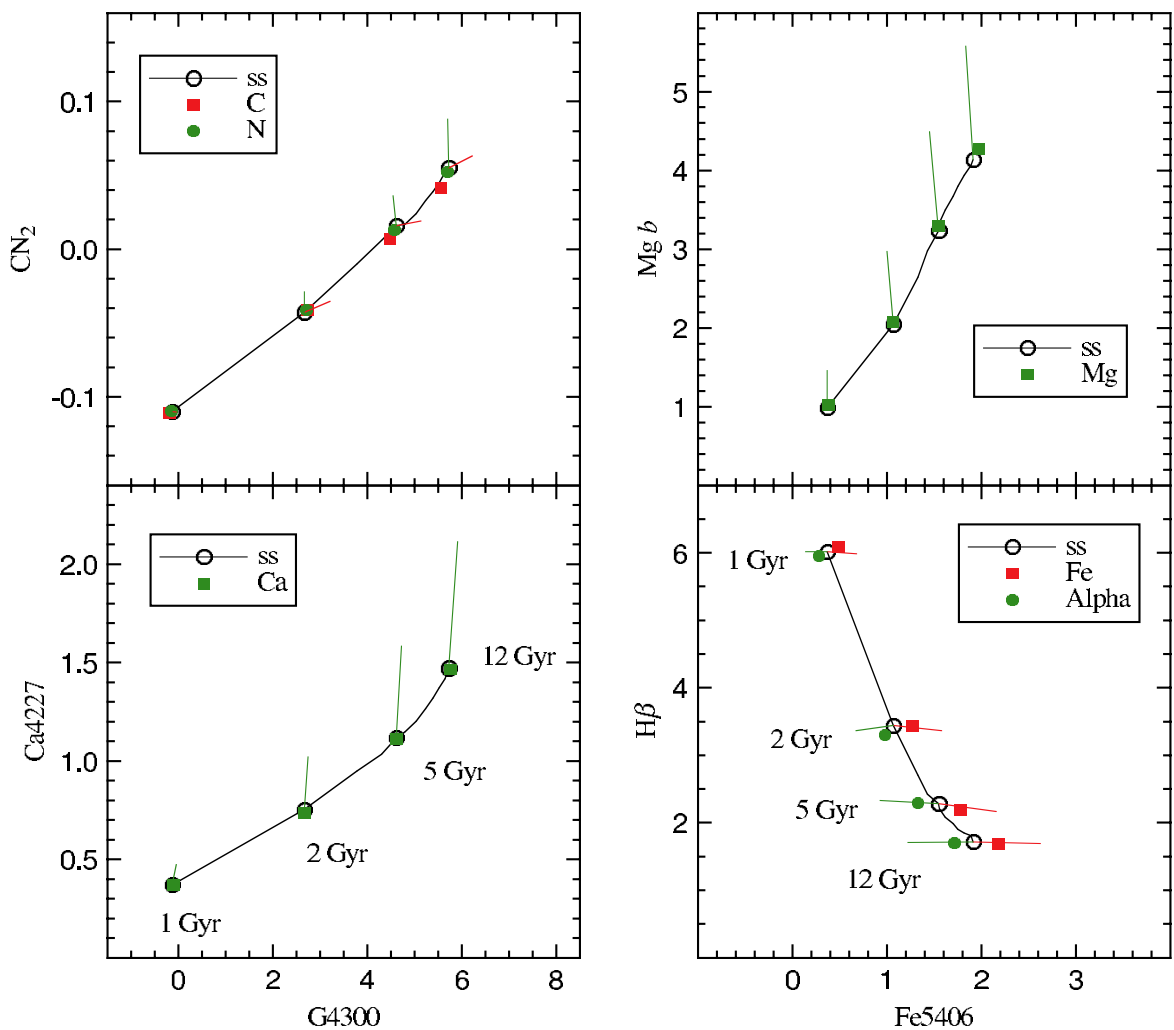

Figure 20. Same as Figure 19, but now the filled symbols denote isochrone effects alone, while the lines show the combination of isochrone and stellar spectral effects. It is clear from this figure that stellar spectral effects dominate the changes in the indices, whereas the isochrone effects are comparatively insignificant (see the text). (A color version of this figure is available in the online journal.) 


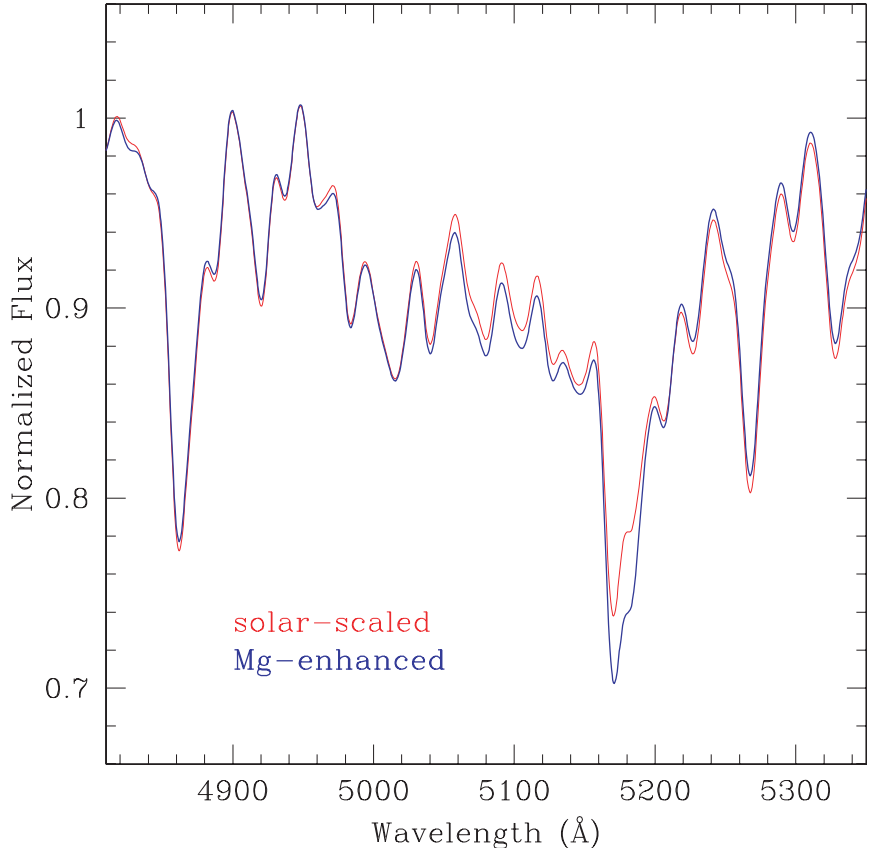

Figure 21. 2 Gyr old, scaled-solar spectrum smoothed to $300 \mathrm{~km} \mathrm{~s}^{-1}$ compared with a 2 Gyr old, +0.3 dex magnesium-enhanced spectrum with the same $Z$ in the SAURON spectral range. The spectra are normalized at $4750 \AA$. Note that $\mathrm{Mg}_{1}(\sim 5100 \AA)$ and $\mathrm{Mg} b(\sim 5175 \AA)$ become stronger with magnesium enhancement.

(A color version of this figure is available in the online journal.)

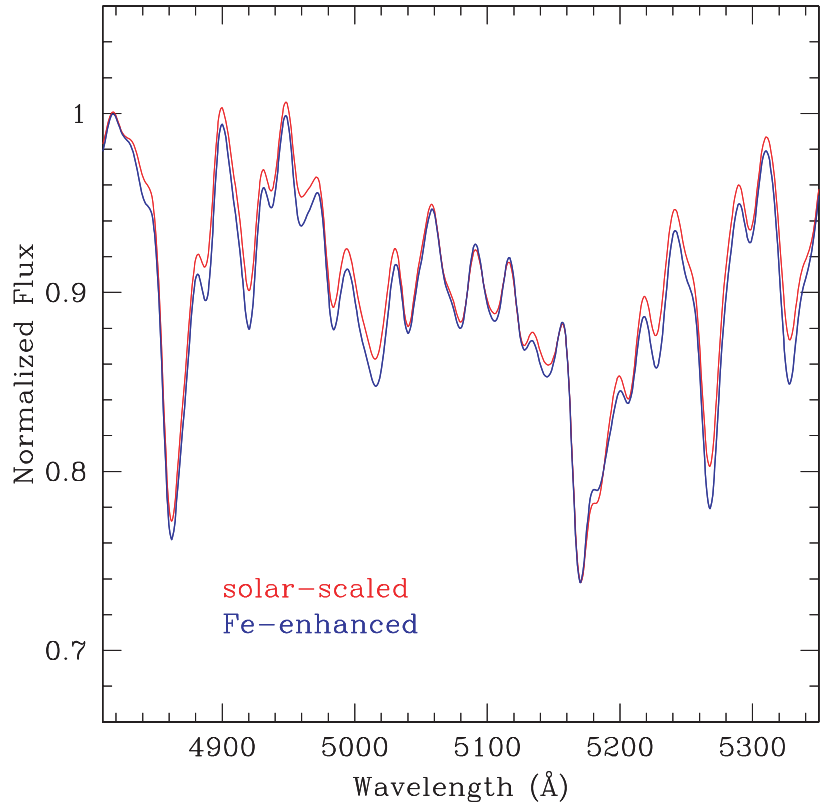

Figure 22. 2 Gyr old, scaled-solar spectrum smoothed to $300 \mathrm{~km} \mathrm{~s}^{-1}$ compared with a 2 Gyr old, +0.3 dex iron-enhanced spectrum with the same $Z$ in the SAURON spectral range. The spectra are normalized at $4750 \AA$. Note that Fe5015, Fe5270, and Fe5335 become stronger with iron enhancement

(A color version of this figure is available in the online journal.)

\section{REFERENCES}

Bacon, R., et al. 2001, MNRAS, 326, 23

Bell, R. A., Paltoglou, G., \& Tripicco, M. J. 1994, MNRAS, 268, 771 Bessell, M. S. 1990, PASP, 102, 1181

Carretta, E., Gratton, R. G., Bragaglia, A., Bonifacio, P., \& Pasquini, L. 2004, A\&A, 416, 925

Cassisi, S., Salaris, M., Castelli, F., \& Pietrinferni, A. 2004, ApJ, 616, 498 Cayrel, R., Perrin, M.-N., Barbuy, B., \& Buser, R. 1991, A\&A, 247, 108

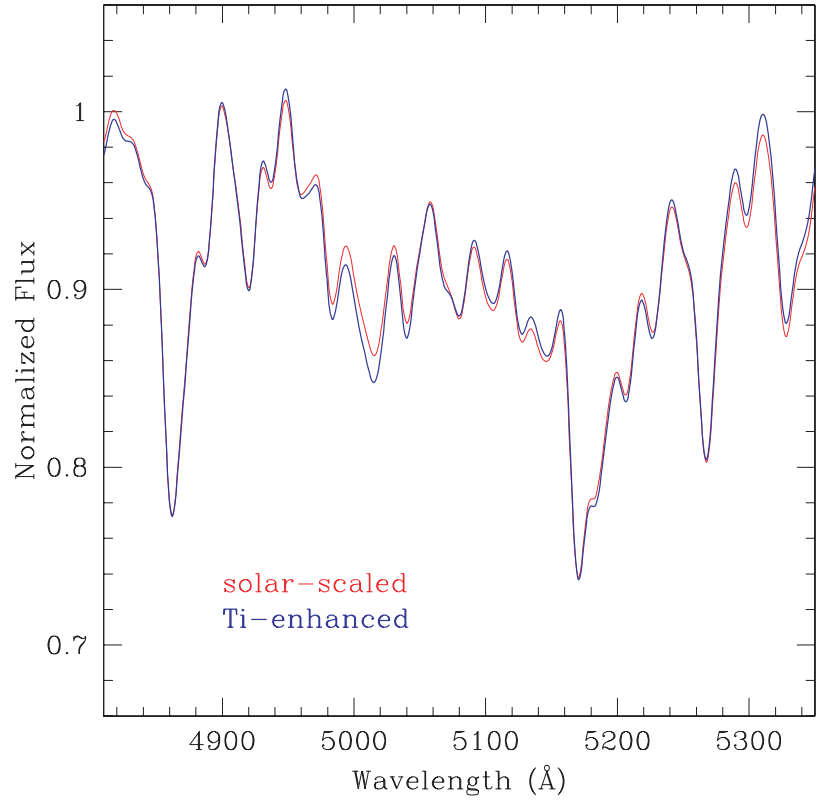

Figure 23. 2 Gyr old, scaled-solar spectrum smoothed to $300 \mathrm{~km} \mathrm{~s}^{-1}$ compared with a 2 Gyr old, +0.3 dex titanium-enhanced spectrum with the same $Z$ in the SAURON spectral range. The spectra are normalized at $4750 \AA$. Note that Fe5015 becomes stronger with titanium enhancement, while the rest of the indices are mostly intact.

(A color version of this figure is available in the online journal.)

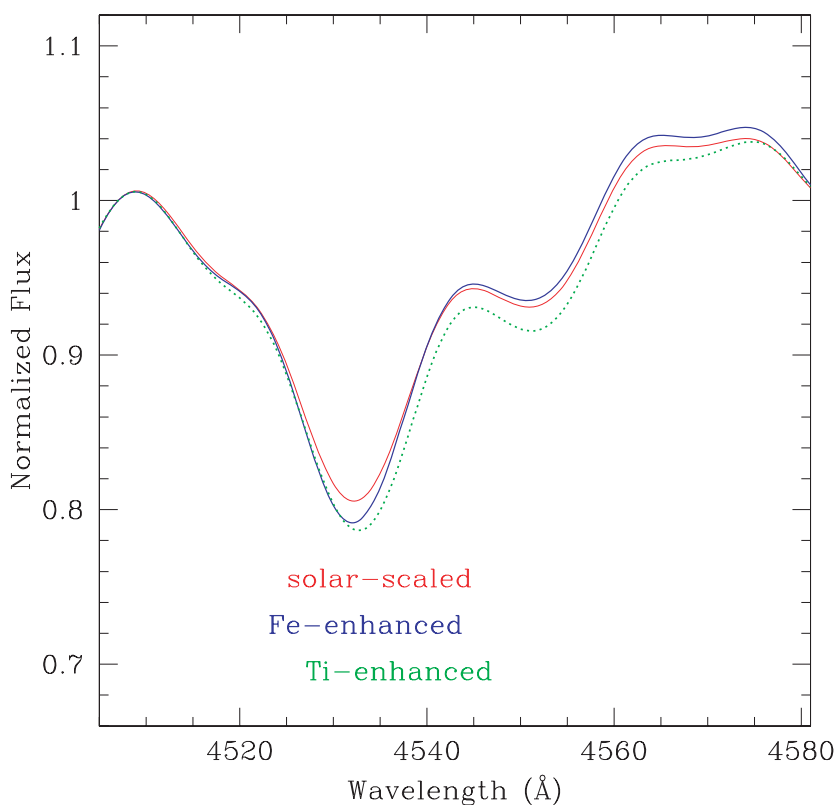

Figure 24. Solar-scaled, Fe-enhanced, and Ti-enhanced 12 Gyr integrated spectra in the Fe4531 index region. Note that the centroids of Fe-enhanced and Ti-enhanced (dotted line) spectra are located to the left and right sides of the solar-scaled one, respectively, because of different locations of $\mathrm{Fe}$ and $\mathrm{Ti}$ lines within the Fe4531 index bandpass.

(A color version of this figure is available in the online journal.)

Charlot, S., Worthey, G., \& Bressan, A. 1996, ApJ, 457, 625

Cid Fernandes, R., et al. 2008, arXiv:0802.0849

Cid Fernandes, R., Mateus, A., Sodre, L., Stasinska, G., \& Gomes, J. M. 2005, MNRAS, 358, 363

Coelho, P., Barbuy, B., Meléndez, J., Schiavon, R. P., \& Castilho, B. V. 2005, A\&A, 443, 735

Coelho, P., Bruzual, G., Charlot, S., Weiss, A., Barbuy, B., \& Ferguson, J. W. 2007, MNRAS, 382, 498

Cohen, J. G., Blakeslee, J. P., \& Ryzhov, A. 1998, ApJ, 496, 808 
Dotter, A., Chaboyer, B., Ferguson, J. W., Lee, H.-c., Worthey, G., Jevremović, D., \& Baron, E. 2007a, ApJ, 666, 403 (Paper I)

Dotter, A., Chaboyer, B., Jevremović, D., Baron, E., Ferguson, J. W., Sarajedini, A., \& Anderson, J. 2007b, AJ, 134, 376

Ferguson, J. W., Alexander, D. R., Allard, F., Barman, T., Bodnarik, J. G., Hauschildt, P. H., Heffner-Wong, A., \& Tamanai, A. 2005, ApJ, 623, 585

Fulbright, J. P., McWilliam, A., \& Rich, R. M. 2007, ApJ, 661, 1152

Graves, G. J., \& Schiavon, R. P. 2008, ApJS, 177, 446

Gray, D. F., \& Brown, K. 2001, PASP, 113, 723

Griffin, R. E. M., \& Lynas-Gray, A. E. 1999, AJ, 117, 2998

Gustafsson, B., Bell, R. A., Eriksson, K., \& Nordlund, Å 1975, A\&A, 42, 407

Koch, A., \& McWilliam, A. 2008, AJ, 135, 1551

Korn, A. J., Maraston, C., \& Thomas, D. 2005, A\&A, 438, 685 (KMT05)

Kurucz, R. L. 1970, Atlas: A Computer Program for Calculating Model Stellar Atmospheres, SAO Special Report 309 (Cambridge: Smithsonian Astrophysical Observatory)

Lee, H.-c., \& Worthey, G. 2005, ApJS, 160, 176 (LW05)

Lee, H.-c., Worthey, G., Trager, S. C., \& Faber, S. M. 2007a, ApJ, 664, 215

Lee, H.-c., et al. 2007b, in IAU Symp. 241, Stellar Populations as Building Blocks of Galaxies, ed. R. F. Peletier, \& A. Vazdekis, (Dordrecht: Kluwer), in press (arXiv:astro-ph/0701666)

Marmol-Queralto, E., et al. 2008, A\&A, 489, 885

Martell, S. L., Smith, G. H., \& Briley, M. M. 2008, PASP, 120, 839

Martins, L. P., \& Coelho, P. 2007, MNRAS, 381, 1329

Milone, A., Barbuy, B., \& Schiavon, R. P. 2000, AJ, 120, 131

Ocvirk, P., Pichon, C., Lancon, A., \& Thiebaut, E. 2006, MNRAS, 365, 74

Panter, B., Jimenez, R., Heavens, A. F., \& Charlot, S. 2007, MNRAS, 378, 1550
Peterson, R. C., Dalle Ore, C. M., \& Kurucz, R. L. 1993, ApJ, 404, 333 Plez, B. 1992, A\&AS, 94, 527

Prochaska, L. C., Rose, J. A., Caldwell, N., Castilho, B. V., Concannon, K., Harding, P., Morrison, H., \& Schiavon, R. P. 2007, AJ, 134, 321

Prochaska, L. C., Rose, J. A., \& Schiavon, R. P. 2005, AJ, 130, 2666

Proctor, R. N., \& Sansom, A. E. 2002, MNRAS, 333, 517

Puzia, T. H., et al. 2002, A\&A, 395, 45

Salasnich, B., Girardi, L., Weiss, A., \& Chiosi, C. 2000, A\&A, 361, 1023

Salpeter, E. E. 1955, ApJ, 121, 161

Schiavon, R. P. 2007, ApJS, 171, 146

Schiavon, R. P., Faber, S. M., Castilho, B. V., \& Rose, J. A. 2002, ApJ, 580, 850

Serven, J. L., Worthey, G., \& Briley, M. M. 2005, ApJ, 627, 754

Thomas, D., \& Maraston, C. 2003, A\&A, 401, 429

Thomas, D., Maraston, C., \& Bender, R. 2003, MNRAS, 339, 897

Thomas, D., Maraston, C., \& Korn, A. J. 2004, MNRAS, 351, L19

Trager, S. C. 1997, PhD thesis, Univ. California, Santa Cruz

Trager, S. C., Faber, S. M., Worthey, G., \& González, J. J. 2000a, AJ, 119, 1645

Trager, S. C., Faber, S. M., Worthey, G., \& González, J. J. 2000b, AJ, 120, 188

Tripicco., M. J., \& Bell, R. A. 1995, AJ, 110, 3035 (TB95)

Vazdekis, A. 1999, ApJ, 513, 224

Weiss, A., Salaris, M., Ferguson, J. W., \& Alexander, D. R. 2006, arXiv:astro-ph/0605666

Worthey, G. 1994, ApJS, 95, 107

Worthey, G. 1998, PASP, 110, 888

Worthey, G., Faber, S. M., González, J. J., \& Burstein, D. 1994, ApJS, 94, 687 Worthey, G., \& Lee, H.-c. 2008, ApJS, submitted (arXiv:astro-ph/0604590)

Worthey, G., \& Ottaviani, D. L. 1997, ApJS, 111, 377

Yong, D., Grundahl, F., Johnson, J. A., \& Asplund, M. 2008, ApJ, 684, 1159 